ELECTRO-RESPONSIVE HYDROGELS

FOR VASCULAR TISSUE ENGINEERING 
Copyright @ Nastaran Rahimi, Maastricht 2014

All rights reserved. No part of this book may be reproduced, stored in a database or retrieval system, or published, in any form or in any way, electronically, mechanically, by print, photoprint, microfilm or any other means without prior written permission by the author.

Cover and Layout: Nastaran Rahimi

Printed by: Proefschriftmaken.nl || Uitgeverij BOXPress

ISBN: 978-94-6295-032-0 


\section{ELECTRO-RESPONSIVE HYDROGELS \\ FOR VASCULAR TISSUE ENGINEERING}

\section{DISSERTATION}

To obtain the degree of Doctor at Maastricht University, on the authority of the Rector Magnificus, Prof. dr. L.L.G. Soete, in accordance with the decision of the board of Deans, to be defended in public on Thursday $18^{\text {th }}$ December 2014, at 10:00 hours

by

Nastaran Rahimi 


\section{Supervisor}

Prof. Dr. Mark. J. Post

\section{Co-supervisor}

Dr. Daniel. G. Molin

\section{Assessment Committee}

Prof. dr. L.Koole (chairman)

Prof. dr. C. Bouten (Eindhoven University of Technology)

Prof. dr. C. Grandfils ( University of Liege, Belgium)

Dr. F. van Nieuwenhoven

Dr. L. Schurgers

This research forms part of the Project P1.01 iValve of the research program of the BioMedical Materials institute, co-funded by the Dutch Ministry of Economic Affairs. The research described in this thesis was supported by a grant of the Dutch Heart Foundation (DHF-2008T089).

Financial support by the Dutch Heart Foundation for the publication of this thesis is gratefully acknowledged.

This research is supported by the Interreg IVa Euregio funded by the dutch heart foundation Meuse-Rhine project BioMIMedics (www.BioMIMedics.org).

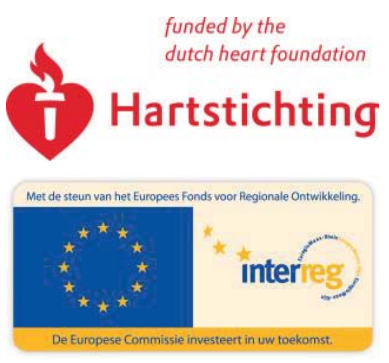




\section{Table of contents}

$\begin{array}{lll}\text { Chapter } 1 & \text { General introduction } & 7\end{array}$

Chapter 2 Electro-sensitive Polyacrylic Acid/Fibrin Hydrogel facilita tes cell seeding and alignment

Chapter 3 Short stimulation of electro-responsive PAA/Fibrin 53 hydrogel inducescollagen production

Chapter 4 Modulation of smooth musc le cells phenotype 79 in an electro-responsive hydrogel

Chapter 5 Vascular cell response in electro-responsive 101 hybrid vasculargraft by short stimulation: in vivo study

Chapter 6 General disc ussion

Appendix 


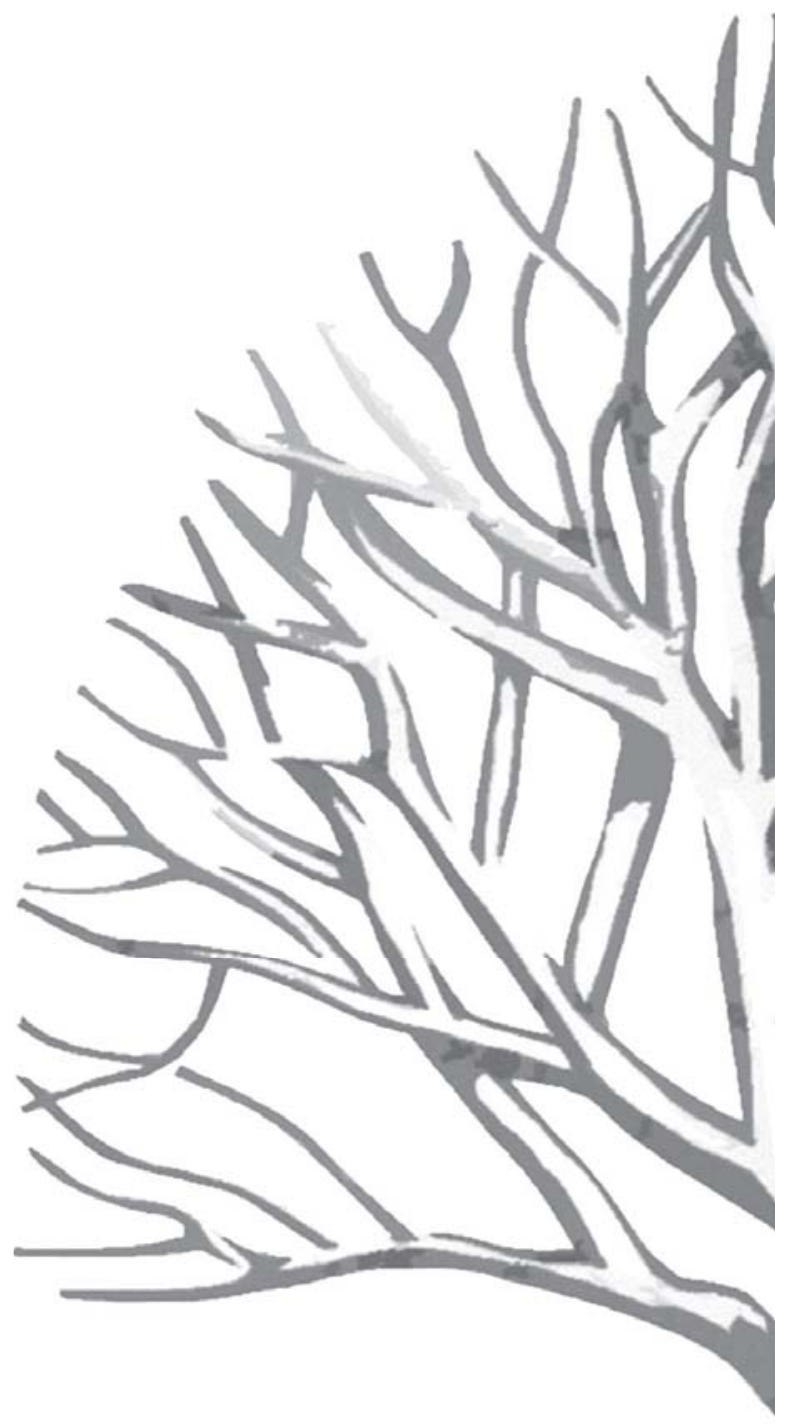




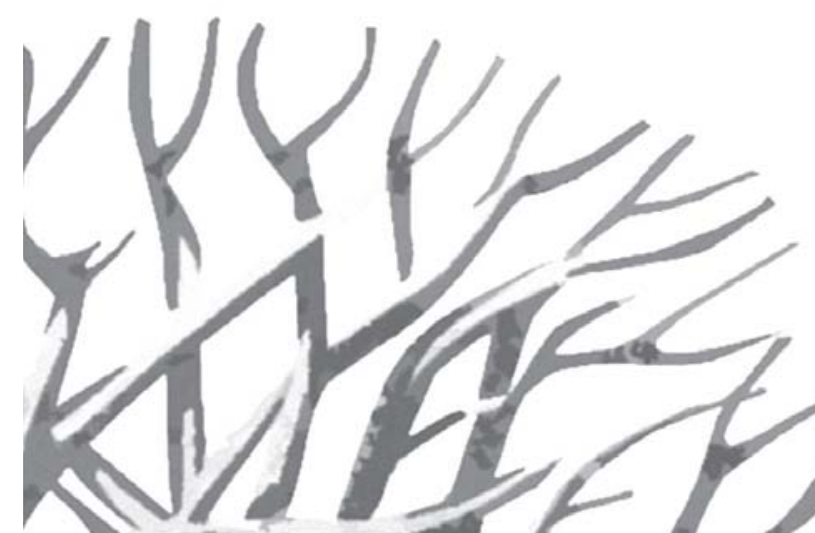




\section{Vascular tissue engineering}

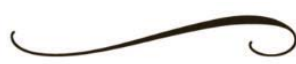

Rapid spreading of vascular diseases in most countries has generated the need to develop suitable replacement materials by vascular tissue engineering [1]. Tissue-engineering implies integration of engineering and life science methodologies to develop a biological substitute to restore, maintain, and potentially improve tissue functions. There is a vast number of investigations on vascular prostheses for replacement of natural blood vessels since 1950 s [2]. One of the earliest approaches used porous woven fabrics of Vinyon- $\mathrm{N}$ and was introduced by Vorhees et al. in 1952 as a promising approach for developing a non-thrombogenic surface that would function as an arterial prosthesis. Since then a variety of materials including Teflon, Orlon and polyurethane were developed and studied [3, 4]. Clinically successful applied synthetic grafts have been designed with diameters above $6 \mathrm{~mm}$, using polyethylene terephthalate (PET) and expanded polytetrafluroethylene (ePTFE) [5, 6]. In spite of many attempts and genuine progress, the development of small-diameter $(<6 \mathrm{~mm})$ artificial vascular grafts with long term patency, low thrombogenicity and good compliance match remains challenging [7].

To obtain a successful tissue engineered cellular vascular substitute it is essential to develop a convenient and reproducible system to adequately control cell behavior during engineering of the cellular component in the graft. Since the early days, scaffolds, cells and signals (i.e. mechanical, chemical) were the major key players in the conceptual framework of tissue engineering (Fig. 1).

\section{Desired cell behavior in tissue engineered vascular grafts}

Vascular cells are continuously exposed to biomechanical forces such as blood shear stress, oscillatory strain and pressure waveforms in vivo. During embryogenesis, angiogenesis and vascular remodeling in later life, cells organize in the most functional configuration to adapt to physical stimuli [8]. The inner layer of blood vessels (intima layer) is made by endothelial cells (ECS), which are aligned longitudinally with the flow direction mainly due to ECs response to fluid shear stress [9]. In tissue engineered vascular grafts not 
only the endothelial cell coverage at the luminal side of the grafts is needed but also the endothelial cells need to display quiescent properties with appropriate function to maintain long term patency of the grafts [10, 11]. Endothelium coverage is critical for preservation of blood flow as it provides an anti-thrombogenic surface, which prevents platelet and leukocyte adhesion $[12,13]$. In addition, ECs can also directly regulate smooth muscle cells by secreting important biomacromolecules such as endothelin and biominimolecules like nitric oxide (NO) [14].

Smooth muscle cells (SMCs) align circumferentially in arranged layers and, especially in arteries, form the media layer of the vessel wall. During in vivo vessel development, SMCs are responsible for secreting Extracellular matrix (ECM) components in the media layer. They undergo a phenotype transition by expressing contractile proteins in support of their function to contract and dilate blood vessels. These phenotypic phases of SMCs are termed "synthetic" and "contractile", respectively [15]. Matrix synthesis and cell proliferation are more indicative of synthetic phenotype while expression of contractile protein and markers are related to the contractile phenotype [16]. Essentially all vascular SMCs have the ability to shift phenotypes, which could be used to advantage in engineering vascular tissue constructs [15]. The alignment of vascular SMCs serves their important role in adjusting the vessel response to the cyclic stretch that results from pulsatile blood flow and pressure [8]. The contraction and dilation of the vessel wall during systolic and diastolic cycles is dampened either by intrinsic elasticity in large arteries or by vascular SMCS contraction in small diameter vessels. Achieving the native cell morphology and alignment as well as adequate cell density in vascular grafts in vivo is one of the most challenging achievements in vascular tissue engineering.

\section{Contribution of collagen}

The mechanical strength of a vessel wall is predominantly provided by ECM components [17]. Collagen is found abundantly in vascular ECM and is mostly produced by smooth muscle cells and fibroblasts in the media and adventitia of the vessel wall. The most common collagens in blood vessels are collagen type I and III. Collagen type I consists of two different genes, $\alpha_{1}(I)$ and $\alpha_{2}(I)$, whereas collagen III has only one gene $a_{1}$ (III). Collagen is the load-bearing element in the vessel wall and plays a critical role in biomechanical properties of the vessel including elongation and distension by accommodating and limiting forces under pressure via its fibers. It also can attach to smooth 
muscle cells and regulate the force around vessel walls [18]. Collagen content, orientation, type and thickness of the fibers are the main properties that adjust the mechanical function of collagen [19].

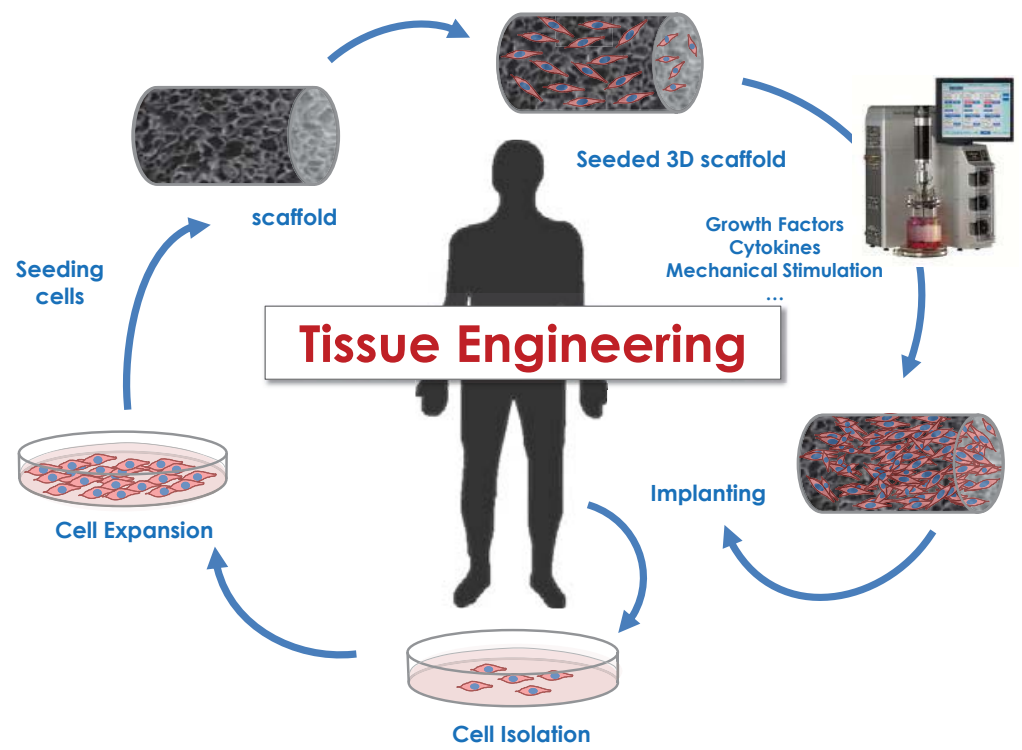

Fig 1. Tissue engineering paradigm; Cells are isolated from a human or -for research purpose- animal body, expanded by cell culture and subsequently seeded on scaffolds and cultured ex vivo under specific cell growth conditions in a bioreactor to mature. During this culture step, cells will produce extracellular matrix (ECM) and form tissue within the scaffold structure. Eventually, the developed tissue engineered graft can be implanted back in patients or animals.

Tissue-engineered grafts often lack sufficient organized content of ECM. Synthesis, accumulation and organization of collagen regulate tissue growth and ensure a mechanically functional engineered graft with appropriate strength. The dynamic balance between collagen production and degradation determines collagen content. The remodeling of the collagen network occurs either by de novo collagen synthesis and/or reorganization of the existing collagen [19]. Matrix metalloproteases (MMP) are a family of proteases responsible for degradation of basal membrane proteins and tissue remodeling. MMP-2 and MMP-9 are two major MMP members produced by 
smooth muscle cells, which participate in vascular remolding as well as collagen breakdown [20].

\section{Biomaterials for vascular tissue engineering}

Biomaterials play an important role in tissue engineering by providing a threedimensional synthetic structure. One of the main approaches in vascular tissue engineering to create new tissue is a $3 \mathrm{D}$ synthetic biomaterial structure that supports cell attachment, uniform distribution of cells and in-growth. In addition, the 3D structure should also provide an appropriate microenvironment to control cell function by incorporating inductive signals for cell migration, proliferation, differentiation, orientation and extracellular matrix (ECM) formation [21].

A vascular substitute needs to have several biological and mechanical properties to perform well in the circulatory system. The material should be biocompatible and leak-proof, it should not cause inflammation or immunogenic responses, should be non-thrombogenic and act as a proper supporter for cells. The material should also provide sufficient mechanical properties including flexibility, strength, resistance to kinking and squashing, ability to stretch, and come with correct compliance and elasticity to mimic the mechanical and viscoelastic nature of an artery or vein, and withstand physiological vascular shear and pressure load. In addition, easy handling and manufacturing of the scaffolds are also recommended to fit the grafts and meet commercial demands [4, 22, 23].

Both natural and synthetic polymers have been widely used for vascular tissue engineering applications. Intrinsic characteristics of natural polymers, such as remodeling and degradation by biological enzymes and their non-toxic and non-immunogenic properties, make them suitable materials for developing vascular scaffolds [24-26]. However, it should be mentioned that regardless of all their advantages they lack sufficient physical and chemical properties to allow easy modification [24, 27].

Beside natural biomaterials, biocompatible synthetic polymers have also been considered as attractive groups of materials for developing scaffolds. The major advantage of synthetic scaffolds is the ability to control their chemical, architectural and mechanical properties during manufacturing of scaffold constructs [28]. These properties include molecular weight, shape, porosity, microstructure, tensile strength and degradation time. These scaffolds can also 
be modified by incorporating natural polymers (e.g. Fibrin) into their structure to improve cell-attachment as well as biological activity [29].

\section{Hydrogels as biomaterials}

Hydrogels are a promising group of biomaterials for tissue engineering and are defined as three-dimensional cross-linked network of polymers. The hydrogel structure typically contains a multidirectional extension of polymer chains produced through polymerization of monomers or by covalent bonding between polymer chains in presence of a crosslinking agent. [30]. Both natural derived hydrogels such as collagen, gelatin, Fibrin, alginate and chitosan as well as synthetic polymer based hydrogel including member of the poly-acrylic acid family, polyethylene glycol, polyvinyl alcohol, polyethylene oxide have been designed for biological applications and drug delivery systems [25, 26, 3133].

Hydrogels could provide a suitable microenvironment for cells by retaining water and nutrients within their three dimensional network due to their hydrophilic nature. Thus, they can be used to mimic the ECM, support cell encapsulation and regulate cell function, i.e. enhance cell proliferation, differentiation and ECM production [34, 35]. Figure 2 shows a schematic overview of a poly-anionic hydrogel structure, including polymer chains as solid phase, an interstitial fluid-filled region and the cations as part of the fluid phase.

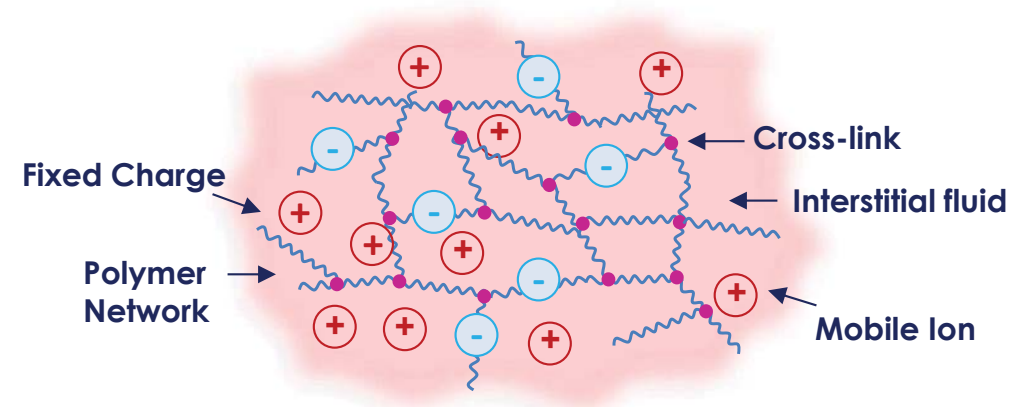

Fig 2. Micro-structure of a poly-anionic hydrogel composed of insoluble cross-linked polymer network with fixed-negatively charged groups and positive mobile ions.

One of the most interesting characteristics of hydrogels is their ability to respond to environmental stimuli such as $\mathrm{pH}$, temperature, ionic strength, 
enzymes, light intensity and electrical field [36-38] (Fig. 3). These hydrogels are defined as smart materials that can undergo continuous or discontinuous conformational changes such as swelling, shrinking, alteration in hydrophilichydrophobic balance or changes to gel-solid phase or even degradation in response to external stimuli [34, 37]. The conformational changes of the hydrogel are the result of interactions between the polymer chain segments or between the polymer network and the solvent molecules, and in most cases these deformations are reversible [39, 40]. The response of hydrogels to external stimuli is mainly determined by the nature of the monomer, density of interactive ionizable charges, pendant chains and the cross-linking degree [41]. The magnitude of the response is also proportional to the applied external stimulus [42].

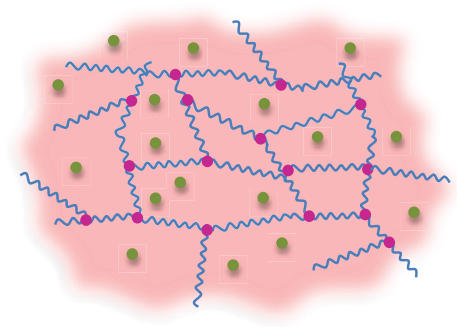

Change in $\mathrm{pH}$, temperature, ionic strength, etc

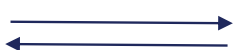

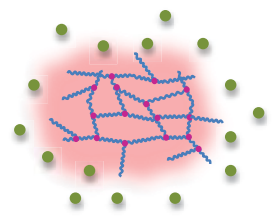

Fig 3. Effect of external stimuli on changing physical properties (shrinking-swelling) of smart hydrogels leading to release or uptake of bioactive molecules (green dots). Pink dots represent cross-linking sites between polymer chains (blue).

Using hydrogels as smart biomaterials for cell culture and tissue formation, an electrical stimulus is the most promising approach as other signals such as $\mathrm{pH}$ and temperature encounter limitations due to the limited physiological range suitable for cells to function properly.

\section{Electro-responsive hydrogels}

Electro-responsive hydrogels hold great potential for significant biological applications in drug release and tissue engineering. They are usually made of poly-electrolytes. When a hydrogel with a poly-electrolyte network consisting of high concentration of ionizable groups along its backbone chain is exposed to an electrical field, a force on counter ions and immobile charged groups is 
produced in the network, which attracts mobile ions to the electrodes. As a result, the hydrogel can swell and shrink regionally at the cathode and anode side respectively, which leads to bending of a one-side mounted hydrogel. This phenomenon is caused by ion concentration differences inside the hydrogel and culture medium and explained by Flory's theory of osmotic pressure [43, 44]. The extent of bending can be controlled by hydrogel formulation and electrical field characteristics including strength, direction and duration of the stimulus. Electrically activated hydrogels can selectively be permeabilized for specific molecular size and control the water permeability by expanding and contracting in micro-pores size under electrical stimulation [45, 46]. For instance, calcium alginate/poly-acrylic acid composites have been used for pulsatile release of hydrocortisone by inducing hydrogel collapse when exposed to an electrical filed [47]. Hydrogels of carboxylic acid derivatives and acrylamide have been developed as voltage switchable artificial muscle-based device [48].

Electro-responsive hydrogels can also be used as smart substrates for vascular grafts. The capability of hydrogel to respond to an electrical field can be utilized to improve cell behavior and enhance ECM production in the tissueengineered grafts. However for tissue engineering applications, gels need to be mechanically strong to withstand physiological conditions. Thus, for in vivo applications, improving properties of hydrogels by incorporating additional polymers to form functionalized hybrid scaffolds is required.

\section{External signals to improve cell behavior}

Controlling and manipulating cell behavior in favor of cell alignment and sufficient tissue formation are prime goals during the biosynthesis of a vascular substitute. Unwanted heterogeneity in cell distribution and tissue formation for cell-seeded scaffolds, with typically high cell density at the edges and low tissue formation in the scaffold's center are most common problems found in tissue-engineered structures. This problem is predominantly caused by inhomogeneous cell seeding of the 3D scaffold structure and inadequate control of tissue formation during culturing [49].

We postulate that application of external signals could be used to control cell distribution and alignment within the scaffold structure. External signals include physical forces such as mechanical, electrical and magnetic stimulation or chemical stimulus like growth factors, cytokines, etc. [50]. They could 
enhance the deposition and remodeling of the ECM produced by cells and improves structural and functional characteristics of the TE grafts.

Applying electrical stimulation can have a multitude of effects on cell behavior. It is a relatively simple technique, which can be precisely controlled and applied both in vitro and in vivo for $2 \mathrm{D}$ or $3 \mathrm{D}$ cultured cells [51]. Cells can respond to electrical stimulation by changing morphology, enhancing migration, reorganizing the cytoskeleton and up-regulating protein expression [52]. It has been established that an electrical field of 1-2 V/cm can control cell orientation and alignment [53]. Clinically, electrical field-based therapies have been used to accelerate healing and repair of skin wounds and fractured bones $[54,55]$.

Mechanical forces such as mechanical stretch, bending, compression loading, fluid shear stress and fluid pressure can also play a significant role in modulating cell physiology and regulate cell growth, elongation, migration, differentiation and ECM deposition of cells [56-58]. In vivo, SMCs are continuously subjected to cyclic stretch and transmural shear due to pulsatile blood pressure during systolic and diastolic cycles. The ability of smooth muscle cells to sense and respond to mechanical stimuli can be used to improve the mechanical strength and accelerate tissue regeneration [59-63]. For example, it has been shown that the expression of contractile genes and proteins in SMCs can be up-regulated by applying mechanical strain, suggesting a switch to the contractile phenotype, which seems in apparent contrast with increasing ECM synthesis, i.e. a synthetic phenotype [16, 64].

The translation of mechanical stimuli into biochemical processing of ECM involves three potential mechano-sensing mechanisms including the cytoskeletal structure, cellular integrins and stretch-ion channel activation of the SMCs, which are mainly determined by cell-cell and bidirectional cell-matrix interactions $[65,66]$.

Other external stimuli such as a strong magnetic field can also be used as biological systems contain weak magnetic properties. These can be exploited to orient the cells and proteins. It has been shown that a magnetic field has the ability to orient collagen fibers and therefore, guide the alignment of the smooth muscle cells via cell contact guidance through the fibers [67]. 


\section{Design and aim of the study}

The art of tissue engineering lies in designing scaffolds where instructive signals can be used to improve and control cell function such as cell attachment, migration and growth. By combining the advantages of different biomaterials, a functional scaffold can be designed to overcome the limitations of current tissue engineered grafts.

The aim of this thesis was to develop a smart scaffold for vascular tissue engineering based on electro-responsive hydrogels. We hypothesized that the conformational changes in the electro-sensitive hydrogel induced by the electrical field enhances cell behavior and 3D tissue growth (Fig. 4).

Poly-acrylic acid (PAA) is a known anionic polymer used in this study. Acrylic acid is an organic component with the simplest formula of unsaturated carboxylic acids. It contains three carbon atoms in which the double bond is conjugated with the carbonyl of the carboxylic group, $\mathrm{CH}_{2}=\mathrm{CHCOOH}$. Acrylic acid derivatives are applicable in a wide range of medical applications [68, 69]. Poly-acrylic acid is produced by covalent linking of Acrylic acid monomers. Due to the presence of carboxylic group, poly-acrylic acid has a high charge density and therefore is well-known to be an electro-responsive polymer [70]. Crosslinked poly-acrylic acid has been used extensively in drug delivery systems [41]. By applying the electrical field, the fixed carboxylic groups as well as other ions in the network generate an osmotic pressure resulting in a regular physical deformation and mechanical stress in the hydrogel as described earlier in the electro-responsive hydrogels section, page 13 [71].

In order to improve the bioactivity and cell adhesion of the PAA hydrogels, natural Fibrin was incorporated into the structure.

Fibrin is a natural biopolymer, which plays an important role in cell and tissue function. It is formed by enzymatic polymerization of Fibrinogen and acts as a bridging molecule for a variety of cell types. In vivo, Fibrin forms a provisional matrix at the sites of injury, inflammation or infection and facilitates tissue regeneration by promoting proliferation, migration and organization of variety of cells including ECs, SMCs, fibroblasts, leukocytes, and keratinocytes [72]. Fibrin can act as reservoir for cytokines and also direct cell signaling through its integrin-mediated mechanisms [73].

Fibrin is increasingly being used in tissue engineering applications. The integrin and growth factor binding sites of Fibrin improve the cell-biomaterial interactions in the scaffold thereby enhancing biological activity [74]. 
Combining PAA with Fibrin results in a scaffold with sufficient structural support for cells, yet the application of PAA/Fibrin hydrogel as an in vivo vascular graft has been limited due to its low mechanical properties. Therefore, we used PAA as coating for electrospun Polycaprolactone (PCL) scaffolds, which have sufficient mechanical strength to serve as vascular grafts. $P C L$ grafts were thus functionalized and used for in vivo implantation.

Polycaprolactone $(P C L)$ is a semi-crystalline bioresorbable synthetic polymer belonging to the aliphatic polyester family. $\mathrm{PCL}$ is non-toxic, inexpensive and easily processed due to its low melting temperature $\left(58-63^{\circ} \mathrm{C}\right)$. Combining these intrinsic features of PCL with the properties of its fibrous form to be water resistant, biodegradable, mechanically strong and highly porous, makes it an excellent choice for tissue engineering. PCL is mostly used as an electrogravity spun scaffold, which can provide heavily interconnected pores along with microscale fibers [75-77].

Incorporation of bioactive biomaterials can improve the biological properties of the structure to a suitable candidate to support cell growth in vascular reconstruction and grafts.

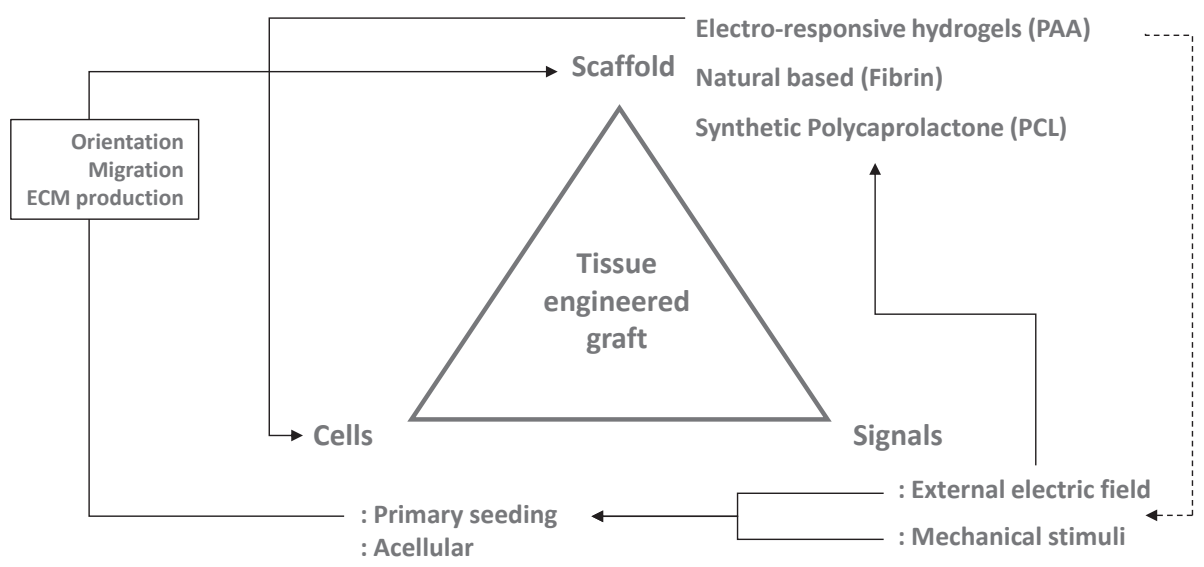

Fig 4. Schematic overview of preparing a tissue engineered graft based on cells, scaffold and signals. Exposing electro-responsive PAA hydrogels to an external electrical field produces a mechanical force in the structure that provides signals to cells and alters their function. 


\section{Overview of the thesis}

In chapter 2, I describe the synthesis, preparation and characterization of the electro-sensitive polyacrylic acid/Fibrin hydrogel and evaluate cell and fiber alignment, as well as cell penetration and distribution in the hydrogels after electrical stimulation.

In chapter 3 the effect of stimulation on tissue growth in the PAA/Fibrin Hydrogels is established by evaluating ECM gene expression, collagen accumulation and reorganization in engineered tissue. The results of tissue formation are verified by performing physical (compaction, bending) and mechanical measurements (stress-strain) of the optimally stimulated engineered tissue.

In chapter 4 the effect of mechanical and electrical stimulation of PAA/Fibrin hydrogels and Fibrin gels on regulation of SMCs phenotype is studied. The FlexCell ${ }^{\circledR}$ system is used to simulate mechanical forces produced by the PAA/Fibrin hydrogel during electrical stimulation. The effect of hydrogel topography on cell behavior and modulation when exposed to external signals is studied.

In chapter 5 we present data on in vivo implanted and electrically stimulated polyacrylic acid hydrogel coated PCL grafts. The patency of grafts after 4 and 8 weeks of follow up is evaluated by recording ultrasound during implantation period and performing angiography just before explant. Histological analyses of the explanted grafts are performed to evaluate cell behavior and effect of stimulation in the grafts.

Chapter 6 summarizes the overall project and discusses pertinent observation with reference to existing evidence. 


\section{References}

1. Sarkar, S., G.Y. Lee, J.Y. Wong, and T.A. Desai, Development and characterization of a porous micro-patterned scaffold for vascular tissue engineering applications. Biomaterials, 2006. 27(27): p. 4775-82.

2. Voorhees, A.B., Jr., A. Jaretzki, 3rd, and A.H. Blakemore, The use of tubes constructed from vinyon "N" cloth in bridging arterial defects. Annals of surgery, 1952. 135(3): p. 332-6.

3. Schmedlen, R.H., W.M. Elbjeirami, A.S. Gobin, and J.L. West, Tissue engineered smalldiameter vascular grafts. Clin Plast Surg, 2003. 30(4): p. 507-17.

4. Greenwald, S.E. and C.L. Berry, Improving vascular grafts: the importance of mechanical and haemodynamic properties. The Journal of Pathology, 2000. 190(3): p. 292-299.

5. Chlupac, J., E. Filova, and L. Bacakova, Blood vessel replacement: 50 years of development and tissue engineering paradigms in vascular surgery. Physiological research / Academia Scientiarum Bohemoslovaca, 2009. 58 Suppl 2: p. S119-39.

6. Kannan, R.Y., H.J. Salacinski, K. Sales, P. Butler, and A.M. Seifalian, The roles of tissue engineering and vascularisation in the development of micro-vascular networks: a review. Biomaterials, 2005. 26(14): p. 1857-75.

7. Baguneid, M.S., A.M. Seifalian, H.J. Salacinski, D. Murray, G. Hamilton, and M.G. Walker, Tissue engineering of blood vessels. The British journal of surgery, 2006. 93(3): p. 282-90.

8. Standley, P.R., A. Camaratta, B.P. Nolan, C.T. Purgason, and M.A. Stanley, Cyclic stretch induces vascular smooth muscle cell alignment via NO signaling. American Journal of Physiology - Heart and Circulatory Physiology, 2002. 283(5): p. H1907H1914.

9. Schwartz Martin A, o.H., Mechanotransduction in Vascular Physiology and Atherogenesis. Nature Reviews Molecular Cell Biology, 2009. 10(1): p. 53-62.

10. Remy-Zolghadri, M., J. Laganiere, J.F. Oligny, L. Germain, and F.A. Auger, Endothelium properties of a tissue-engineered blood vessel for small-diameter vascular reconstruction. Journal of vascular surgery, 2004. 39(3): p. 613-20.

11. Li, S. and J.J. Henry, Nonthrombogenic approaches to cardiovascular bioengineering. Annual review of biomedical engineering, 2011. 13: p. 451-75.

12. Luscher, T.F. and M. Barton, Biology of the endothelium. Clinical cardiology, 1997. 20(11 Suppl 2): p. II-3-10.

13. Xiupeng, W., H. Fupo, L. Xia, I. Atsuo, S. Yu, M. Osamu, K. Ryo, and Y. Jiandong, Tissue-engineered endothelial cell layers on surface-modified Ti for inhibiting in vitro platelet adhesion. Science and Technology of Advanced Materials, 2013. 14(3): p. 035002. 
14. Stankevicius, E., E. Kevelaitis, E. Vainorius, and U. Simonsen, [Role of nitric oxide and other endothelium-derived factors]. Medicina, 2003. 39(4): p. 333-41.

15. Stegemann, J.P., H. Hong, and R.M. Nerem, Mechanical, biochemical, and extracellular matrix effects on vascular smooth muscle cell phenotype. Journal of applied physiology, 2005. 98(6): p. 2321-7.

16. Stegemann, J. and R. Nerem, Phenotype Modulation in Vascular Tissue Engineering Using Biochemical and Mechanical Stimulation. Annals of Biomedical Engineering, 2003. 31(4): p. 391-402.

17. Sarkar, S., T. Schmitz-Rixen, G. Hamilton, and A. Seifalian, Achieving the ideal properties for vascular bypass grafts using a tissue engineered approach: a review. Medical \& Biological Engineering \& Computing, 2007. 45(4): p. 327-336.

18. Bou-Gharios, G., M. Ponticos, V. Rajkumar, and D. Abraham, Extra-cellular matrix in vascular networks. Cell proliferation, 2004.37(3): p. 207-20.

19. Holzapfel, G.A., Collagen in Arterial Walls: Biomechanical Aspects, in Collagen: Structure and Mechanics, P. Fratzl, Editor 2008. p. 285-324.

20. Johnson, C. and Z.S. Galis, Matrix metalloproteinase-2 and -9 differentially regulate smooth muscle cell migration and cell-mediated collagen organization. Arteriosclerosis, Thrombosis, and Vascular Biology, 2004. 24(1): p. 54-60.

21. Juliana Lott Carvalho, P.H.d.C., Dawidson Assis Gomes and Alfredo Miranda de Goes, Innovative Strategies for Tissue Engineering, in Advances in Biomaterials Science and Biomedical Applications. R. Pignatello, Editor 2013, InTech. p. 295-313.

22. Thomas, A.C., G.R. Campbell, and J.H. Campbell, Advances in vascular tissue engineering. Cardiovascular Pathology, 2003. 12(5): p. 271-276.

23. Venkatraman, S., F. Boey, and L.L. Lao, Implanted cardiovascular polymers: Natural, synthetic and bio-inspired. Progress in Polymer Science, 2008. 33(9): p. 853-874.

24. Dang, J.M. and K.W. Leong, Natural polymers for gene delivery and tissue engineering. Adv Drug Deliv Rev, 2006. 58(4): p. 487-99.

25. Mano, J.F., G.A. Silva, H.S. Azevedo, P.B. Malafaya, R.A. Sousa, S.S. Silva, L.F. Boesel, J.M. Oliveira, T.C. Santos, A.P. Marques, et al., Natural origin biodegradable systems in tissue engineering and regenerative medicine: present status and some moving trends. Journal of the Royal Society, Interface / the Royal Society, 2007. 4(17): p. 999-1030.

26. Malafaya, P.B., G.A. Silva, and R.L. Reis, Natural-origin polymers as carriers and scaffolds for biomolecules and cell delivery in tissue engineering applications. Advanced Drug Delivery Reviews, 2007.59(4-5): p. 207-33.

27. Vara, D.S., H.J. Salacinski, R.Y. Kannan, L. Bordenave, G. Hamilton, and A.M. Seifalian, Cardiovascular tissue engineering: state of the art. Pathologie Biologie, 2005. 53(10): p. 599-612.

28. Ravi, S. and E.L. Chaikof, Biomaterials for vascular tissue engineering. Regenerative medicine, 2010. 5(1): p. 107-20. 
29. Shaikh, F.M., A. Callanan, E.G. Kavanagh, P.E. Burke, P.A. Grace, and T.M. McGloughlin, Fibrin: a natural biodegradable scaffold in vascular tissue engineering. Cells Tissues Organs, 2008. 188(4): p. 333-46.

30. Polymer Grafting and Crosslinking, in Polymer Grafting and Crosslinking, J.W.R. Amit Bhattacharya, Paramita Ray, Editor 2009, John Wiley \& Sons, Inc.,: New Jersey.

31. Lee, K.Y. and D.J. Mooney, Hydrogels for tissue engineering. Chem Rev, 2001. 101(7): p. 1869-79.

32. Hoare, T.R. and D.S. Kohane, Hydrogels in drug delivery: Progress and challenges. Polymer, 2008. 49(8): p. 1993-2007.

33. Vermonden, T., R. Censi, and W.E. Hennink, Hydrogels for protein delivery. Chem Rev. 112(5): p. 2853-88.

34. Kopecek, J., Hydrogel biomaterials: a smart future? Biomaterials, 2007. 28(34): p. 5185-92.

35. Drury, J.L. and D.J. Mooney, Hydrogels for tissue engineering: scaffold design variables and applications. Biomaterials, 2003. 24(24): p. 4337-51.

36. Jeong, B. and A. Gutowska, Lessons from nature: stimuli-responsive polymers and their biomedical applications. Trends in Biotechnology, 2002. 20(7): p. 305-311.

37. Qiu, Y. and K. Park, Environment-sensitive hydrogels for drug delivery. Advanced Drug Delivery Reviews, 2001. 53(3): p. 321-339.

38. de Las Heras Alarcon, C., S. Pennadam, and C. Alexander, Stimuli responsive polymers for biomedical applications. Chemical Society reviews, 2005. 34(3): p. 276-85.

39. Melekaslan, D. and O. Okay, Swelling of strong polyelectrolyte hydrogels in polymer solutions: effect of ion pair formation on the polymer collapse. Polymer, 2000. 41(15): p. 5737-5747.

40. Furth, M.E., A. Atala, and M.E. Van Dyke, Smart biomaterials design for tissue engineering and regenerative medicine. Biomaterials, 2007. 28(34): p. 5068-73.

41. Murdan, S., Electro-responsive drug delivery from hydrogels. Journal of controlled release : official journal of the Controlled Release Society, 2003. 92(1-2): p. 1-17.

42. Soppimath, K.S., T.M. Aminabhavi, A.M. Dave, S.G. Kumbar, and W.E. Rudzinski, Stimulus-responsive "smart" hydrogels as novel drug delivery systems. Drug Dev Ind Pharm, 2002. 28(8): p. 957-74.

43. Yao, K. and S. Sun, Bending behavior of an electrically activated complex forming hydrogel. Polymer Bulletin, 1992. 28(6): p. 677-681.

44. Shiga, T. and T. Kurauchi, Deformation of polyelectrolyte gels under the influence of electric field. Journal of Applied Polymer Science, 1990. 39(11-12): p. 2305-2320.

45. Yoshihito osada, M.H. Electrically activated mechanochemical devices using polyelectrolyte gels. Chemistry Letters 1985; 1285-1288].

46. Garland, M.J., T.R.R. Singh, A.D. Woolfson, and R.F. Donnelly, Electrically enhanced solute permeation across poly(ethylene glycol)-crosslinked poly(methyl vinyl ether-comaleic acid) hydrogels: Effect of hydrogel crosslink density and ionic conductivity. International Journal of Pharmaceutics, 2011. 406(1-2): p. 91-98. 
47. Yuk, S., S. Cho, and H. Lee, Electric Current-Sensitive Drug Delivery Systems Using Sodium Alginate/Polyacrylic Acid Composites. Pharmaceutical Research, 1992. 9(7): p. 955-957.

48. Moschou, E.A., M.J. Madou, L.G. Bachas, and S. Daunert, Voltage-switchable artificial muscles actuating at near neutral pH. Sensors and Actuators B: Chemical, 2006. 115(1): p. 379-383.

49. Melchels, F.P., A.M. Barradas, C.A. van Blitterswijk, J. de Boer, J. Feijen, and D.W. Grijpma, Effects of the architecture of tissue engineering scaffolds on cell seeding and culturing. Acta biomaterialia, 2010. 6(11): p. 4208-17.

50. Nemeno-Guanzon, J.G., S. Lee, J.R. Berg, Y.H. Jo, J.E. Yeo, B.M. Nam, Y.G. Koh, and J.I. Lee, Trends in tissue engineering for blood vessels. Journal of biomedicine \& biotechnology, 2012. 2012: p. 956345.

51. Sun, S., I. Titushkin, and M. Cho, Regulation of mesenchymal stem cell adhesion and orientation in 3D collagen scaffold by electrical stimulus. Bioelectrochemistry, 2006. 69(2): p. 133-41.

52. Rowlands, A.S. and J.J. Cooper-White, Directing phenotype of vascular smooth muscle cells using electrically stimulated conducting polymer. Biomaterials, 2008. 29(34): p. 4510-20.

53. Li, X. and J. Kolega, Effects of direct current electric fields on cell migration and actin filament distribution in bovine vascular endothelial cells. Journal of vascular research, 2002. 39(5): p. 391-404.

54. Balakatounis, K.C. and A.G. Angoules, Low-intensity electrical stimulation in wound healing: review of the efficacy of externally applied currents resembling the current of injury. Eplasty, 2008. 8: p. e28.

55. R Onibere, A.K., The Role Of Electrical Stimulation In Fracture Healing. The Internet Journal of Orthopedic Surgery, 2008. 11.

56. Butler, D.L., S.A. Goldstein, and F. Guilak, Functional tissue engineering: the role of biomechanics. Journal of biomechanical engineering, 2000. 122(6): p. 570-5.

57. Brown, T.D., Techniques for mechanical stimulation of cells in vitro: a review. Journal of biomechanics, 2000. 33(1): p. 3-14.

58. Seliktar, D., R.M. Nerem, and Z.S. Galis, Mechanical strain-stimulated remodeling of tissue-engineered blood vessel constructs. Tissue engineering, 2003. 9(4): p. 657-66.

59. Hahn, M.S., Chapter 7: Mechanical Stimulation and Biomimetic Scaffolds for Tissue Engineered Vascular Grafts, in Topics in tissue engineering2008.

60. Liu, B., M.J. Qu, K.R. Qin, H. Li, Z.K. Li, B.R. Shen, and Z.L. Jiang, Role of cyclic strain frequency in regulating the alignment of vascular smooth muscle cells in vitro. Biophysical journal, 2008. 94(4): p. 1497-507.

61. Kim, B.S., J. Nikolovski, J. Bonadio, and D.J. Mooney, Cyclic mechanical strain regulates the development of engineered smooth muscle tissue. Nature biotechnology, 1999. 17(10): p. 979-83. 
62. Sharifpoor, S., C.A. Simmons, R.S. Labow, and J.P. Santerre, A study of vascular smooth muscle cell function under cyclic mechanical loading in a polyurethane scaffold with optimized porosity. Acta biomaterialia, 2010. 6(11): p. 4218-28.

63. Wang, C., L. Cen, S. Yin, Q. Liu, W. Liu, Y. Cao, and L. Cui, A small diameter elastic blood vessel wall prepared under pulsatile conditions from polyglycolic acid mesh and smooth muscle cells differentiated from adipose-derived stem cells. Biomaterials, 2010. 31(4): p. 621-30.

64. Rensen, S.S., P.A. Doevendans, and G.J. van Eys, Regulation and characteristics of vascular smooth muscle cell phenotypic diversity. Netherlands heart journal : monthly journal of the Netherlands Society of Cardiology and the Netherlands Heart Foundation, 2007. 15(3): p. 100-8.

65. Bishop, J.E. and G. Lindahl, Regulation of cardiovascular collagen synthesis by mechanical load. Cardiovascular research, 1999. 42(1): p. 27-44.

66. Dado, D. and S. Levenberg, Cell-scaffold mechanical interplay within engineered tissue. Seminars in Cell \& Developmental Biology, 2009. 20(6): p. 656-664.

67. Tranquillo, R.T., T.S. Girton, B.A. Bromberek, T.G. Triebes, and D.L. Mooradian, Magnetically orientated tissue-equivalent tubes: application to a circumferentially orientated media-equivalent. Biomaterials, 1996. 17(3): p. 349-357.

68. Scranton, A.B., B. Rangarajan, and J. Klier, Biomedical applications of polyelectrolytes, in Biopolymers II, N. Peppas and R. Langer, Editors. 1995, Springer Berlin Heidelberg. p. 1-54.

69. Ohara, T., T. Sato, N. Shimizu, G. Prescher, H. Schwind, O. Weiberg, K. Marten, and H. Greim, Acrylic Acid and Derivatives, in Ullmann's Encyclopedia of Industrial Chemistry2000, Wiley-VCH Verlag GmbH \& Co. KGaA.

70. Kim, S.J., K.J. Lee, and S.I. Kim, Water sorption of poly(propylene glycol)/poly(acrylic acid) interpenetrating polymer network hydrogels. Reactive and Functional Polymers, 2003. 55(1): p. 69-73.

71. Esmaiel, J., T. Javad, and S.S. Alireza, Swelling characteristics of acrylic acid polyelectrolyte hydrogel in a dc electric field. Smart Materials and Structures, 2007. 16(5): p. 1614 .

72. Laurens, N., P. Koolwijk, and M.P. de Maat, Fibrin structure and wound healing. Journal of thrombosis and haemostasis : JTH, 2006. 4(5): p. 932-9.

73. Cornwell, K.G. and G.D. Pins, Discrete crosslinked Fibrin microthread scaffolds for tissue regeneration. Journal of biomedical materials research. Part A, 2007. 82(1): p. 104-12.

74. Lorentz, K.M., S. Kontos, P. Frey, and J.A. Hubbell, Engineered aprotinin for improved stability of Fibrin biomaterials. Biomaterials, 2011. 32(2): p. 430-8.

75. Reed, C.R., L. Han, A. Andrady, M. Caballero, M.C. Jack, J.B. Collins, S.C. Saba, E.G. Loboa, B.A. Cairns, and J.A. van Aalst, Composite tissue engineering on polycaprolactone nanofiber scaffolds. Annals of plastic surgery, 2009. 62(5): p. 505-12. 
76. Tillman, B.W., S.K. Yazdani, S.J. Lee, R.L. Geary, A. Atala, and J.J. Yoo, The in vivo stability of electrospun polycaprolactone-collagen scaffolds in vascular reconstruction. Biomaterials, 2009. 30(4): p. 583-8.

77. Williamson, M.R., R. Black, and C. Kielty, PCL-PU composite vascular scaffold production for vascular tissue engineering: attachment, proliferation and bioactivity of human vascular endothelial cells. Biomaterials, 2006. 27(19): p. 3608-16. 


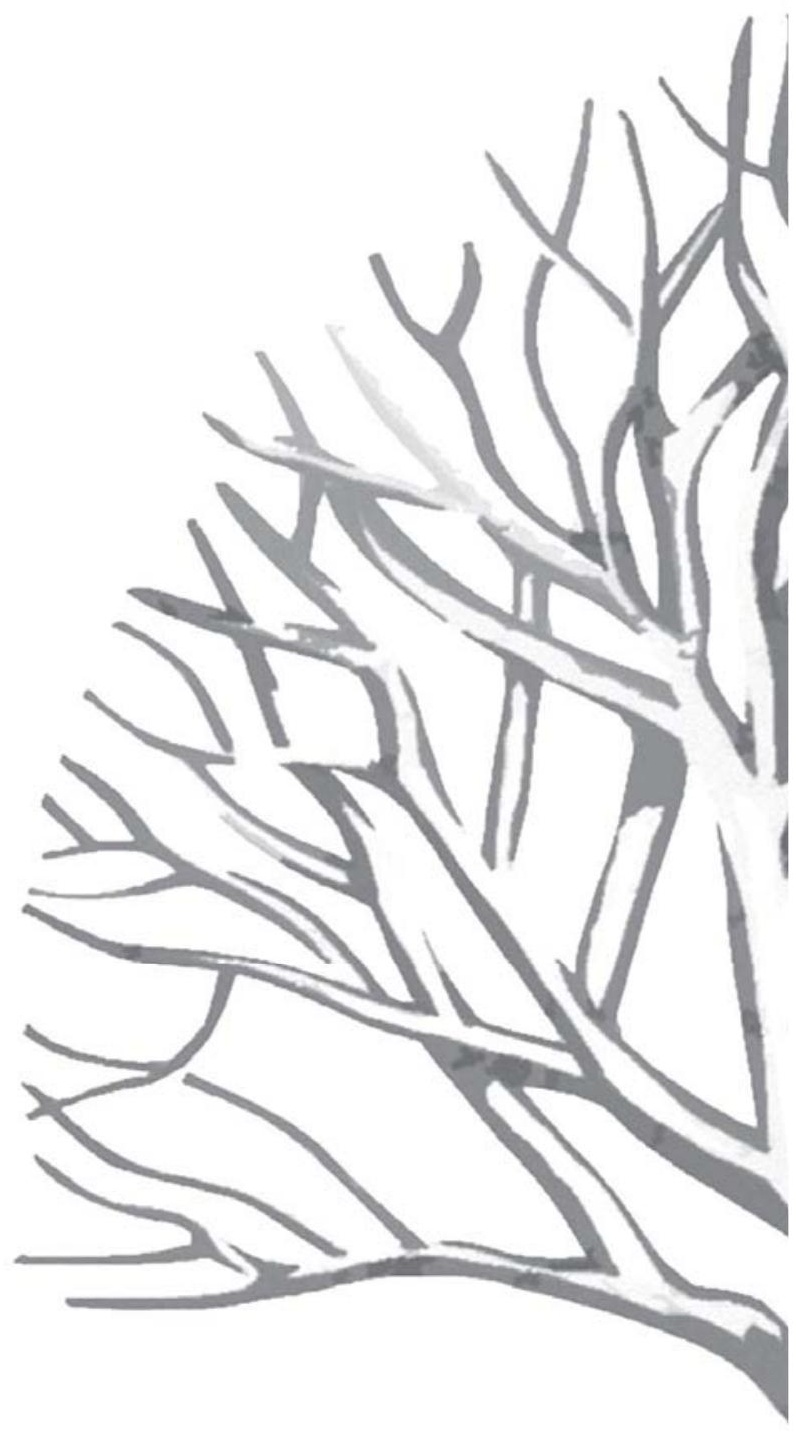




\section{Abstract}

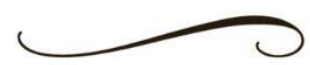

3D Cell culture and conditioning is an effective means to guide cell distribution and patterning for tissue engineered constructs such as vascular grafts. Poly acrylic acid is known as an electro-responsive polymer, capable to transform environmental stimuli like electrical energy to mechanical forces. In this study, we developed an electro-sensitive and biocompatible hydrogel based smart device composed of Acrylic acid and Fibrin as a tissue engineered construct to mechanically stimulate cells. Structural properties of the hydrogel were assessed by FTIR-ATR, scanning electron microscopy, prosimetry and swelling measurement. Distribution and alignment of porcine smooth muscle cells ( $\mathrm{pSMCs}$ ) seeded on the surface of lyophilized hydrogels were evaluated and quantified by two-photon laser scanning microscopy. Smooth muscle cell tissue constructs exposed to 2 hrs of pulsatile electrical stimulation showed significantly enhanced cell penetration and alignment due to dynamic changes produced by alternative swelling and deswelling, in comparison with static samples.

On the basis of the results, this hydrogel under electrical stimulation works as a mechanical pump, which can direct SMC alignment and facilitate infiltration and distribution of cells throughout the structure. 


\section{Introduction}

The endemic nature of vascular diseases and increasing demands for vascular grafts has triggered the search for a suitable replacement through vascular tissue engineering (TE) [1]. Despite many attempts, one common problem in seeded scaffolds is heterogeneity of tissue formation with strong formation at the edges and reduced formation in the center of the tissue. Inhomogeneous seeding and inadequate control of tissue formation during the culturing process are likely the main causes of the heterogeneity. Application of external signals to control and improve the functionality of TE structures might improve cell distribution and alignment, resulting in a better functionality [2-4].

Mechanical forces such as mechanical stretch or compression can regulate cell growth, migration and differentiation [5, 6]. Likewise, electrical stimulation can direct cell elongation and migration [7-9]. TE scaffolds need to conduct the external stimuli while at the same time providing support for the cells.

Hydrogels with their three dimensional network can provide an appropriate microenvironment for cells by retaining water and nutrients, by mimicking the extracellular matrix and by supporting cell proliferation and differentiation [10-13]. One of the most interesting properties of hydrogels is their ability to respond to environmental stimuli such as $\mathrm{pH}$, ionic strength, temperature or electrical field $[14,15]$.

Employing smart biomaterials in medical applications has attracted much attention in recent years because of their controllable and reversible responses to changes in physiological conditions [16-18]. In the polyelectrolyte network of a hydrogel, an electrical field produces a force on counter ions and immobile charged groups in the network and attracts mobile ions to the electrodes. As a result, the hydrogel swells and shrinks regionally, which leads to bending. The extent of bending depends on strength, direction and duration of the electrical stimulus. This bending behavior is explained by Flory's theory of osmotic pressure, and is caused by ion concentration differences inside the hydrogel and culture medium [19-23].

In this study we developed a semi-interpenetrating network (semi-IPN) electro-sensitive hydrogel/Fibrin based smart device to stimulate the TE hydrogel constructs electrically and mechanically. These hydrogels can transform the electrical energy into mechanical work, which is expected to 
improve cell distribution and tissue production within the cultured scaffold after seeding.

\section{Methods}

\section{Materials}

Acrylic acid (AA), purity $99.9 \%$ was purchased from Merck, Germany. N-N Methylenebisacrylamide (MBAA) solution $2 \%$, Ammonium persulfate (APS), purity 99\%, Tetramethylethylenediamine (TMEDA), Fibrinogen and Thrombin from bovine plasma and Verapamil hydrochloride were purchased from Sigma Aldrich, The Netherlands. All chemicals were molecular biology reagents and used without further purification.

\section{Preparation of PAA/Fibrin hydrogel}

The electrical-responsive hydrogel was synthesized by free-radical polymerization and crosslinking of acrylic acid in the presence of Fibrin, using Ammonium persulfate (APS), Tetramethylethylenediamine (TMEDA) and N-N methylenebisacrylamid (MBAA) as initiator, accelerator and crosslinking agent, respectively. AA and MBAA (2 wt.\% of monomer) were dissolved in deionized water at room temperature and stirred. Fibrin was prepared by mixing fibrinogen $(20 \mathrm{mg} / \mathrm{mL})$ and thrombin $(20 \mathrm{IU} / \mathrm{mL})$ and added to the solution. APS and TMEDA ( 0.2 and 0.4 mol.\% of the monomer respectively) were slowly added to the mixture. The solution was stirred continuously for 60 minutes, then molded into 1-mm-thick plate, and heated at $80^{\circ} \mathrm{C}$ for $3 \mathrm{hrs}$. Prepared hydrogels were washed with acetone and immersed in distilled water for 72 hrs to remove residual components. Subsequently, fully swollen hydrogels were first frozen at $-20^{\circ} \mathrm{C}$ for $24 \mathrm{hrs}$ and then freeze-dried (Freeze dryer, Lyovac GT 2) under vacuum pressure of $4.5 \times 10-2$ mbar at $-35^{\circ} \mathrm{C}$ for 48 hrs.

\section{Hydrogel characterization}

Without disturbing the pore structure, lyophilized hydrogels were fractured using a razor blade to allow analysis by scanning electron microscope (SEM). The cross sectioned samples were sputtered with a thin layer of gold under an 
argon atmosphere (Cressington Sputter Coater 108auto) and scanned by electron microscope (XL-30 Philips) at $10 \mathrm{kV}$. The resulting scanning micrographs were used to determine the morphology and pore size of the structure.

The porosity of freeze-dried hydrogel was measured by solvent replacement method. Dry hydrogels were immersed in absolute ethanol for $24 \mathrm{hrs}$ and excess ethanol was removed by blotting before measuring hydrogel weight. The porosity was calculated as follows:

Porosity $=\left(M_{2}-M 1\right) / \rho V ; M 1$ and $M_{2}$ represent the hydrogel mass before and after immersion in absolute ethanol, respectively; $\rho$ density of absolute ethanol, and $\mathrm{V}$ volume of the sample [24].

The chemical characterization of the hydrogels was performed by using Fourier transform infrared (FT-IR) spectrometer equipped with PIKE MIRacle attenuated total reflection (MIR-ATR) attachment (Bruker, Tensor 27). The absorption spectra of the dry samples were recorded in the frequency range of $800-4000 \mathrm{~cm}-1$ with $4 \mathrm{~cm}-1$ resolution.

To measure the equilibrium water content of the hydrogel (EWC), preweighed freeze-dried samples were immersed in distilled water at $37^{\circ} \mathrm{C}$. The hydrogel mass was measured as a function of time. The excessive water at the surface of the gel was removed by filter paper before each measurement. The procedure was repeated three times. EWC was calculated as follows: Equilibrium water content $=(\mathrm{Wt}-\mathrm{Wd}) \mathrm{Wd}$; where $\mathrm{Wd}$ and $\mathrm{Wt}$ are the weights of the sample in initial dry and swollen state at time point, $t$.

\section{Hydrogel behavior under electric stimulus}

Fully swollen hydrogel strips $(15 \times 4 \times 1 \mathrm{~mm} 3)$ were placed between two carbon electrodes in a culture plate (Nunclon 4-well dish, Ion Optix, USA). The hydrogel was fixed parallel to the electrodes, at one end by tissue glue (Vetbond TM Tissue Adhesive, USA). The space between the electrodes was $80 \mathrm{~mm}$. Direct electrical field of $0.06 \mathrm{~V} \mathrm{~mm}-1$ was applied to the sample, while the voltage polarity was changed every 60 seconds by a programmable switch. The effect of higher amplitude ( 0.0875 and $0.125 \mathrm{~V} \mathrm{~mm}-1)$ and longer duration of polarization ( $5 \mathrm{~min}, 10 \mathrm{~min}$ and direct-current) on bending behavior of hydrogel was also studied. The technical setup and protocol used for bending is shown schematically in Fig. 1. Deformation of the hydrogel strips was recorded every 15 seconds by a camera connected to the microscope (EVOS ${ }^{\circledR}, A M G$, USA). The bending angle $(\theta)$ was determined by 
measuring the deviation of the hydrogel from a fixed vertical middle line as reference. (Fig. 1) The bending angle is considered to be positive when the hydrogel bends toward the upper electrode and negative when it bends toward the lower one.

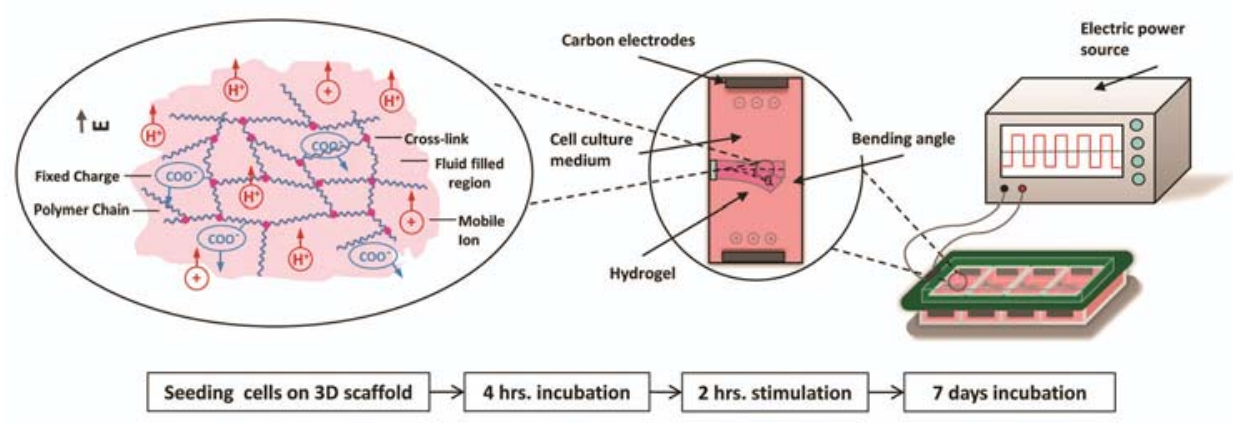

Fig. 1. Schematic of polyelectrolyte hydrogel bending under electrical stimuli. The gel shrinks at its anode side and swells at the cathode side when exposed to electrical field (polarization of voltage changes every $60 \mathrm{sec}$ ). Responsive behavior of hydrogel was measured regarding the bending angle in acellular hydrogels and pSMCs seeded ones stimulated $4 \mathrm{hrs}$ after seeding for $120 \mathrm{~min}$. Cell behavior was evaluated after one week of culture.

\section{Cell culture}

Primary porcine smooth muscle cells (pSMCs) were isolated from pig aorta by standard collagenase digestion. Cells were expanded in fibronectin coated culture plates containing smooth muscle basal medium (SmBM, Lonza) supplemented with $5 \%$ fetal bovine serum (FBS), human epidermal growth factor, human fibroblast growth factor- $B$, insulin and gentamicin. Cells were incubated at $37^{\circ} \mathrm{C}$ under $5 \% \mathrm{CO} 2$; culture medium was changed every two days until cells reached $80-90 \%$ confluence. Cells were harvested by applying 0.5 $\mathrm{g} / \mathrm{mL}$ trypsin (Lonza). pSMCs between passages 8 and 12 were used in this study.

\section{Cell seeded hydrogels}

Freeze-dried hydrogels were sterilized under ultraviolet irradiation in a biological cabinet for 1 hour [25]. Hydrogels were then placed and fixed at one end in a 4 well plate equipped with carbon electrodes. Primary porcine 
smooth muscle cells $\left(5 \times 10^{5}\right.$ pSMCs) suspended in $100 \mu \mathrm{L}$ of culture medium (SmBM) were added to the surface of the freeze dried hydrogel strips ( $15 \mathrm{~mm}$ $\times 4 \mathrm{~mm} \times 1 \mathrm{~mm})$. Seeded hydrogels were incubated $\left(37{ }^{\circ} \mathrm{C}, 5 \% \mathrm{CO} 2\right)$ for 30 minutes to allow initial cell adherence and penetration into the structure, before the addition of culture medium to the wells. Hydrogels were either cultured statically as controls or were stimulated 4 hrs later (after seeding) by exposure to $0.06 \mathrm{~V} \mathrm{~mm}-1$ electrical field; the direction of the current was changed every 60 seconds for 2 hrs of stimulation (at $37^{\circ} \mathrm{C}, 5 \% \mathrm{CO} 2$ ). Next, the cellular hydrogels were cultured for a maximum of 7 days in culture medium (SmBM), with medium changed every two days.

\section{The role of voltage gated $\mathrm{Ca}^{2+}$ channels}

The external electrical field applied to hydrogels can change the membrane potential of cells seeded in the structure. L-type calcium channels are involved in initiation and control of smooth muscle cell contractility and tone. $\mathrm{Ca}^{2+}$ activates the contractile protein and influences the cytoskeleton in the smooth muscle in response to electrical stimulation. In order to block the effect of applied electrical field to the contractile behavior of cells, cells were treated with $10 \mu \mathrm{M}$ verapamil to block L-type calcium channels during the 2 hrs stimulation [26, 27]. Verapamil was washed away from the samples afterward.

\section{MTS assay}

Quantitative assessment of cell viability was evaluated by Promega CellTiter $96{ }^{\circledR}$ AQueous Non-Radioactive Cell Proliferation Assay. The assay consists of colorimetric (3-(4,5-dimethylthiazol-2-yl)-5-(3-carboxymethoxyphenyl)-2-(4sulfophenyl)-2H tetrazo-lium) (MTS), which reduces to Formazan by dehydrogenase enzyme activity of viable cells. Formazan is soluble in cell culture medium and can be measured at $490 \mathrm{~nm}$. This assay was performed on the samples $24 \mathrm{hrs}$ after seeding. To eliminate the effect of phenol red absorbance, smooth muscle basal medium (SmBM, Lonza) was replaced with phenol-red free Dulbecco's modified eagle's medium (DMEM, Gibco, Life science) 4 hrs before running the assay. Briefly, medium was aspirated and gels were washed twice with phosphate buffer solution (PBS 1x) to remove the non-attached cells, next 20\% MTS substrate diluted in DMEM with $2.5 \%$ FBS, was added to the samples followed by 3 hrs incubation at $37^{\circ} \mathrm{C}$ and $1 \mathrm{hr}$ on a shaker to ensure penetration of MTS into the hydrogel. The solution was 
then transferred to a 96 well plate and the absorbance was recorded at 490nm using a plate reader $\left(\mathrm{VICTOR}^{\mathrm{TM}} \mathrm{X}\right.$, Perklin Elmer). A standard absorbance curve was calculated to determine accuracy of the assay.

\section{Microscopy}

Morphology of the non-seeded hydrogel before and after the stimulation was assessed by Fibrin(ogen) staining. Hydrogels were incubated with FITC-labeled anti-fibrinogen antibody (WAK Chemine, Germany) diluted in PBS (1:100). Fluorescent imaging was performed by 2-photon laser scanning microscope (2PLSM, Biorad, Great Britain) coupled to a Nikon E60oFN microscope (Nikon, Tokyo, Japan). The excitation source of the microscope was a 140-fs-pulsed Ti:sapphire laser (Spectra Physics Tsunami, Mountain View, Calif., USA) tuned and mode-locked at $800 \mathrm{~nm}$ (for visualization of fluorescent probes) [28, 29]. For pSMCs seeded constructs, samples were washed with PBS $1 \mathrm{x}$, and incubated in a mixture of SYTO-13 ( $\lambda$ max emission= $510 \mathrm{~nm}$, Invitrogen) greenfluorescent nucleic acid stain and propidium iodide ( $\mathrm{Pl}, \lambda \max$ emission $=620$ $\mathrm{nm}$, Invitrogen) red fluorescent nucleic acid stain for 30 minutes at $37^{\circ} \mathrm{C}$. Syto$13(5 \mathrm{mM})$ and PI were diluted (2:1000 and 5:1000 in PBS, respectively). Samples were scanned for SYTO13/PI to image cell viability seeded in the structure, 1 day after seeding.

To evaluate cell distribution and characteristics, pSMCs seeded constructs that were cultured for 7 days, were fixed in paraformaldehyde $2 \%$ for 20 minutes at $4{ }^{\circ} \mathrm{C}$, washed twice with PBS before they were incubated with alpha-Smooth Muscle - FITC antibody (Sigma) diluted (1:250 in PBS) at $4{ }^{\circ} \mathrm{C}$ overnight and, next, emerged in DAPI solution diluted (1:1000 in PBS) for $15 \mathrm{~min}$ at room temperature. All samples were fixed in $2 \%$ agarose gel prior to visualizing by 2PLSM. For each section ( $n=3$ per group) three parts were chosen for imaging. Sections were recorded in the $x$-y plane with z-steps of $2-4 \mu \mathrm{m}$ into the depth of the construct, starting at upper surface (Z-stack) with a $40 x$ water dipping objective with $2.0 \mathrm{~mm}$ working distance (numerical aperture (NA) 0.8, Nikon) [29]. Density and penetration depth of pSMCs seeded under both static (nonelectro stimulated) and stimulated conditions were evaluated by counting the number of cells in different volume layers within the structure [30]. 2PLSM images collected from hydrogel cross-section (thickness up to $700 \mu \mathrm{m}$ ) were equally divided into groups each contained on average 33 images $(100 \mu \mathrm{m})$ of hydrogel 2D cross-sections ( $x$-y plane). The images were converted to 8-bit to build 3D images by using Volume builder plug-in of ImageJ ( $\mathrm{NIH}$, Bethesda, 
MD, USA). The number of cells in the 3D images was calculated by $3 \mathrm{D}$ Object Counter plug-in ImageJ. Z coded stack plug-in ImageJ was used to adjust color gradient to stained cells along the $Z$ direction. To quantify the projected area, adjusted threshold images were analyzed by using the Area calculator plug-in of ImageJ, The coverage was presented as the total area of the signal above the threshold divided by the total area of image. All settings were kept constant during analysis of all groups.

\section{Statistical analysis}

All quantitative experiments were performed in triplicate and data were expressed as means \pm standard deviation for $n=3$ or more. Statistical analysis of the results was performed either by One-way or Two-way repeated measured ANOVA (Graph pad prism 4) to compare groups multiple means with one or two parameters respectively. Non-parametric Two-sample Kolmogorov-Smirnov Test (K-S) was used to test differences in distribution between group pairs (SPSS 17). P-values <0.05 were considered to be significant in all.

\section{Results and discussion}

\section{Hydrogel structure}

Swelling ratio is an important element that can express hydrogel characteristics such as hydrophilicity and crosslinking ratio as well as available pore space in the structure [31]. The swelling kinetics of prepared hydrogels depended on the amount of acrylic acid and the acrylic acid to Fibrin ratio used whereas monomer to cross-linker ratio was kept constant in all the experiments (Fig. 2a,b). Fibrin is a hydrophobic component in the polymer network, which resulted in decreased swelling of the hydrogel and increased time to reach equilibrium in low concentration (1:1 ratio).

However, as the amount of Fibrin fibers increase in the acrylic acid network (3:1 ratio) it causes an increase in swelling. These results suggest that higher amounts of Fibrin fibers can cause a rearrangement of the hydrogel molecular structure by interfering with acrylic acid crosslinking (Fig. 2a). Most likely the higher Fibrin fibers content in the acrylic acid network reduces the degree of crosslinking between the chains resulting in a loose structure with higher 
swelling capacity. Others have published similar results [32, 33]. Increasing acrylic acid monomer concentration in the feed mixture, with a fixed polymer/Fibrin ratio $(1: 1 \mathrm{w} / \mathrm{w})$ decreased swelling capacity of the hydrogel through increased cross-linking of acrylic chains (Fig. 2b). This reduces chain mobility and results in a rigid structure. Conversely, lower amounts of acrylic acid allow the hydrogel to take up more solution due to less cross-linked chains in the network [34]. However, free carboxylic charged groups in the acrylate polymer chains determine the hydrogel reactivity in an electrical field, which would argue in favor of a higher amount of acrylic acid [35]. To balance between swelling ratio and electro-sensitivity of acrylic acid, a concentration of $11.1 \mathrm{mM}$ was found to be optimal.
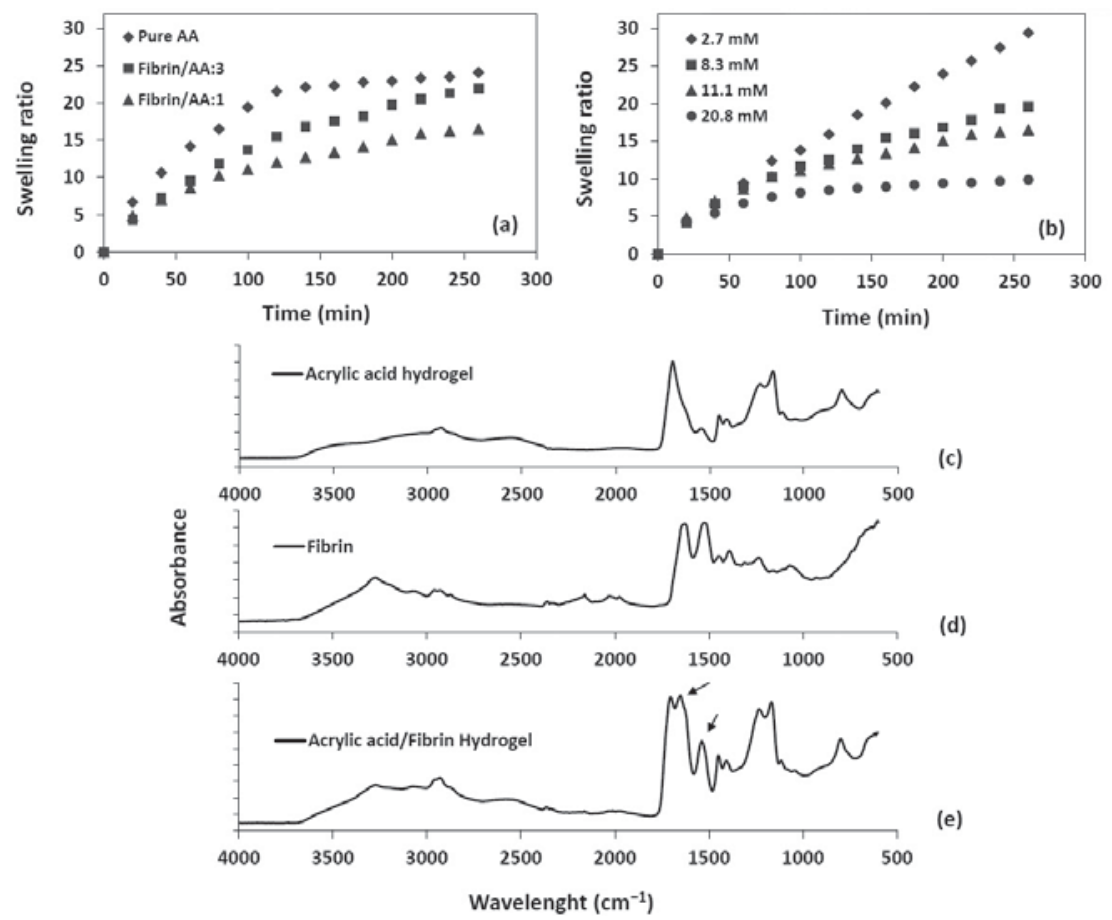

Fig. 2. (a) Effect of Fibrin/ AA ratio on swelling ratio of the hydrogel (b) Effect of monomer concentration (AA) on swelling ratio of the hydrogel, AA/Fibrin: 1:1 (c) FTIRATR spectroscopy of Acrylic acid hydrogel, (d) Fibrin and (e) Acrylic acid/Fibrin hydrogel, arrows at 1665, 1539 indicate shifted bands of amide I and amide II vibrations as a result of Fibrin incorporation into the network. 
The FTIR-ATR spectra of pure polyacrylic acid and polyacrylic acid/Fibrin hydrogel (1:1 w/w) confirmed the presence of Fibrin in the gel (Fig. 2c-e). The broad peaks at 2800 to $3400 \mathrm{~cm}^{-1}$ and at $2947 \mathrm{~cm}^{-1}$ present in all samples are due to $\mathrm{O}-\mathrm{H}$ stretching and $\mathrm{C}-\mathrm{H}$ stretching in $-\mathrm{CH}_{2}$ group, respectively. In Fig. 2C, absorption peaks at 1697 and $1232 \mathrm{~cm}^{-1}$ are attributed to stretching vibration of $\mathrm{C}=\mathrm{O}$ and $\mathrm{C}-\mathrm{O}$ of carboxylate group. The bands at 1410 and 1450 $\mathrm{cm}-1$ are due to $\mathrm{C}-\mathrm{OH}$ bending vibration of PAA [36]. The three dominant bands in Fibrin spectrum at 1631, 1527 and $1238 \mathrm{~cm}^{-1}$ are assigned to amide I, II and III, respectively (Fig. 2d) [37]. Between PAA/Fibrin hydrogel and pure PAA hydrogel, two new peaks at 1665, 1539 (arrows in Fig. 2e) appeared, which can be attributed to shifted bands of amide I and amide II vibrations of proteins in fibrinogen. The shift of the stretching band of $\mathrm{C}=\mathrm{O}$ to $1704 \mathrm{~cm}-1$ and combination of $\mathrm{C}-\mathrm{O}$ and amide III band to a single peak at $1234 \mathrm{~cm}-1$ confirms the incorporation of Fibrin into the acrylic acid network [37, 38].

The SEM micrographs illustrate highly homogenous well-interconnected porosity throughout the scaffold (Fig. 3a) after lyophilization. Fibrin clots and fibers with an average diameter of $137 \pm 42 \mathrm{~nm}$ were oriented along the pore wall surfaces and show the integration of Fibrin in the polyacrylic network (Fig. 3b, c). The average pore size and porosity of the lyophilized hydrogel were $150 \pm 30 \mu \mathrm{m}$ and $88 \pm 6 \%(n=3)$, respectively according to SEM pictures and alcohol porosimetry.
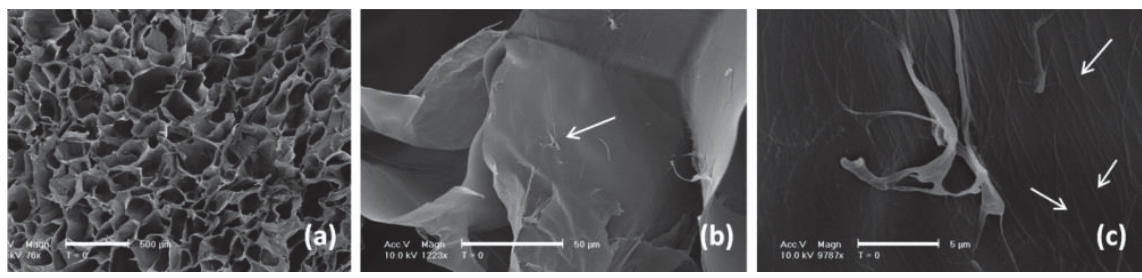

Fig.3. SEM micrographs of freeze-dried PAA/Fibrin hydrogel (a) cross-section (b, c) inner pore surface where Fibrin clot (arrow in b) and Fibrin fibers (arrows in c) can be visualized. The scale bars from panels a to $c$ represent, 500, 50 and $5 \mu \mathrm{m}$ respectively. 
Morphological changes of the hydrogel by electrical stimulation were also visualized with a Fibrin stain. Fluorescent images of labeled fibrin(ogen) shown in black and white (Fig. 4) display high alignment of fibrin(ogen) in the direction of the electrical field, whereas fibers were randomly dispersed in the non-stimulated samples. The same result was obtained at $\mathrm{pH}=6.5$ (Fig. 1 . Supporting information), the isoelectric focus of Fibrin, suggesting that these morphological changes are primarily related to structural alteration of the hydrogel.
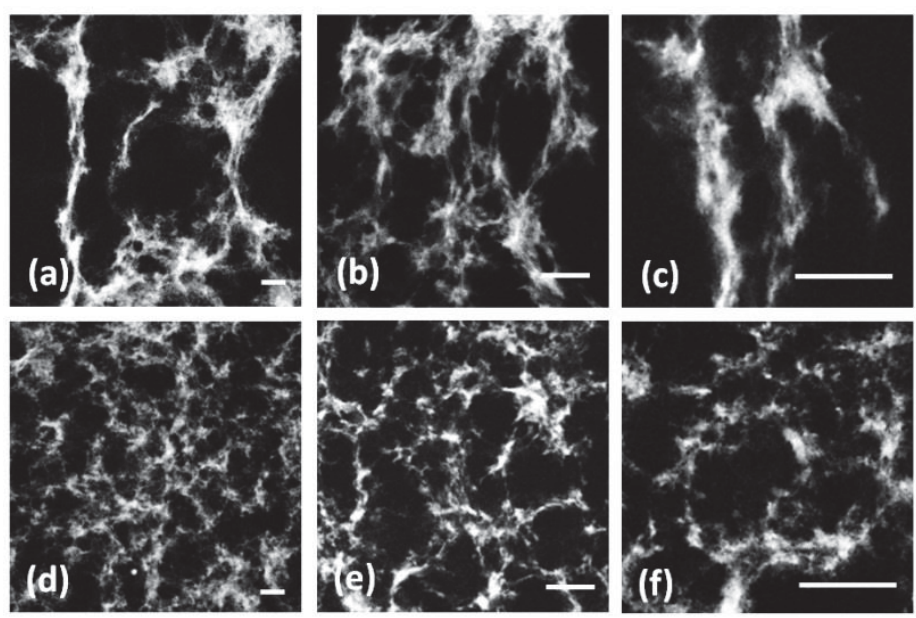

Fig. 4. Morphology of fibrin(ogen) dispersion in PAA/Fibrin hydrogel, stimulated by alternating electrical field of $0.06 \mathrm{~V} \mathrm{~mm}-1$ for 2 hrs (a-c) and non-stimulated condition (d-f), scale bars represents $100 \mu \mathrm{m}$ in all images. The direction of the electrical field was from top to bottom for each slide.

\section{Electro-responsive bending of Polyacrylic acid/Fibrin hydrogel}

Bending of the pre-swelled PAA/Fibin hydrogel strips was examined in smooth muscle basal medium ( $\mathrm{SmBM}$, Lonza) supplemented with $5 \%$ fetal bovine serum (FBS). The hydrogel showed rapid bending toward the cathode side during electrical field application, reaching 1.4, 1.6 and 2.5 degrees deviation in 60 seconds by a $0.06,0.0875$ and $0.125 \mathrm{~V} \mathrm{~mm}-1$, respectively (Fig. 5b). In addition, by increasing the exposure time to 5 and 10 minute, bending of the hydrogel was increased to 5 and 10 degrees, respectively. Maximum bending of the hydrogel was 27 degrees, and was reached after 25 minutes of direct 
electrical current stimulation. Polarity change induced bending of the hydrogel in the opposite direction (video 1, Supplementary data). The mechanism of polyelectrolyte hydrogel deformation under an electrical field is repeatedly caused by swelling or deswelling of certain parts of the gel due to changes in osmotic pressure inside and outside the hydrogel because of charged ion attraction to the anode or cathode sides [23, 39]. The osmotic pressure gradient in turn causes a uni-axial stress along the hydrogel, which is the driving force for the bending.

The result indicates that the magnitude and speed of hydrogel bending were directly related to the electrical field intensity and increased by increasing voltage as has been published by others [40-43]. In order to stimulate cellseeded hydrogels, behavior of SMCs seeded in 2D fibronectin-coated glass cover under applied voltages of $0.06,0.0875$ and $0.125 \mathrm{~V} \mathrm{~mm}^{-1}$ was studied. We observed that SMCs detached immediately from the surface when the voltages applied was higher than $0.0750 \mathrm{~V} \mathrm{~mm}^{-1}$ (data not shown). Furthermore, applying high voltage and direct current for longer time increases the risk of electrolysis in medium. Thus, seeded hydrogels were stimulated at $0.06 \mathrm{~V} \mathrm{~mm}^{-1}$ for 120 minutes after $4 \mathrm{hrs}$ of seeding with a polarization time of 1 minute to make sure that the responsive shape change of the hydrogel stays constant during all cycles of the stimulation. The presence of SMCs did not affect the bending behavior (Fig. $5 \mathrm{C}$ and d).

\section{Cell attachment and viability}

Cell presence and viability one day after seeding was assessed by staining of pSMCs, with the nuclear stain SYTO-13 and propidium iodine (PI; red fluorescent) (Fig. 6a). SYTO-13 can enter both dead and alive cells and thus stain the nucleus of all cells green. In contrast PI can only enter dead cells and binds more strong than SYTO to nucleic acid, results all live cells remain green while dead cells exhibit red nucleus fluorescence, which can be distinguished easily. Cell viability was high in the synthesized hydrogel, which correlates well with the MTS assay that was performed to evaluate the metabolic activity of the cells (Fig. 6b). There was no difference in MTS outcome between the stimulated and non-stimulated constructs (One-Way ANOVA, $p=0.78$ ). We also inferred that the electrical-mechanical forces applied to the seeded cells did not affect their attachment to the polymer network as there was no significant difference between the total cell numbers in the stimulated 
samples compared to non-stimulated ones and 2D cell culture on polystyrene plate as control.
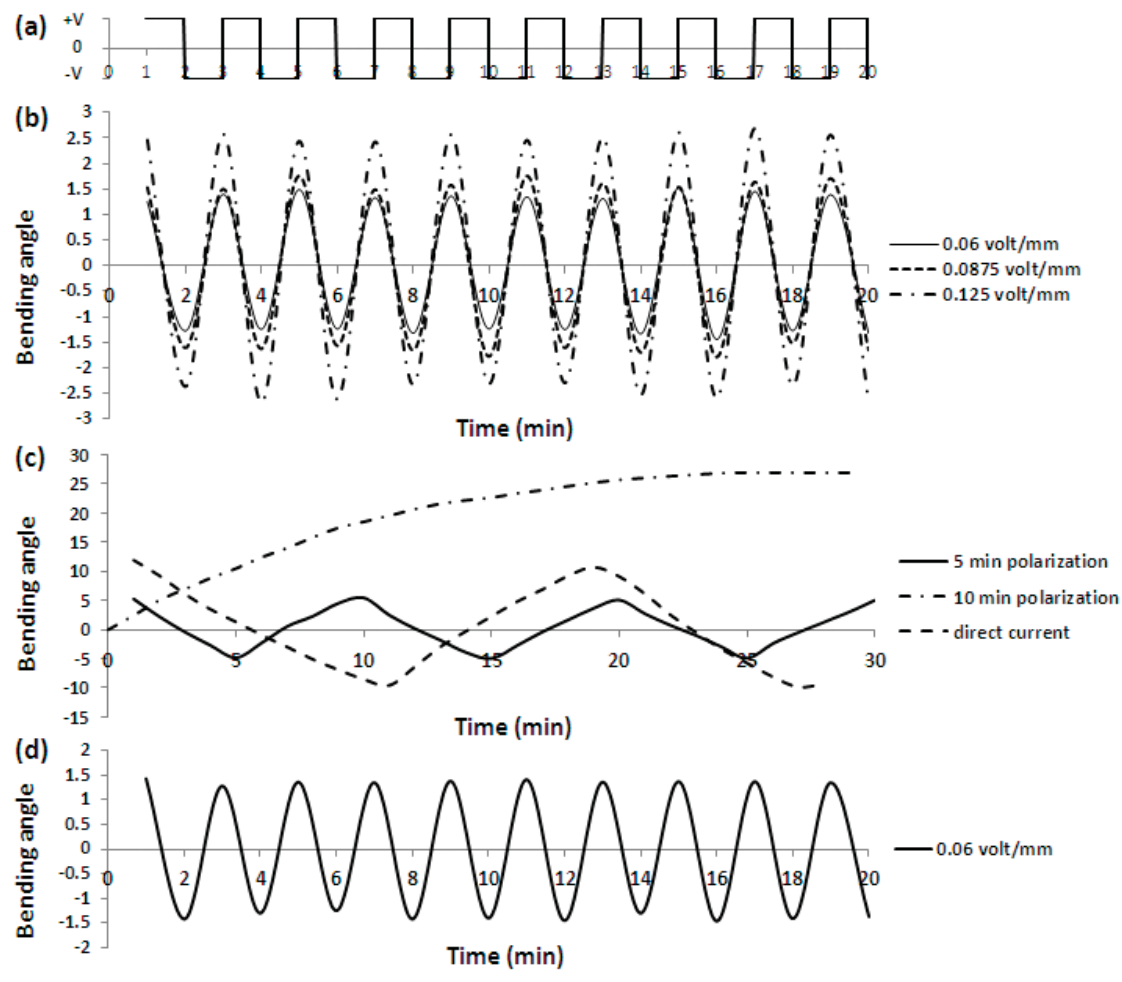

(e)
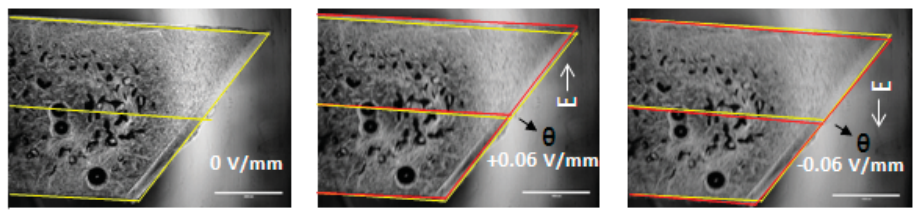

Fig. 5. (a) Applied electrical voltage pattern, changing polarity every 60 seconds, Effect of different electrical voltage (b) and effect of different polarization time (5 min and $10 \mathrm{~min}$ ) and direct electrical field current of $0.06 \mathrm{~V} \mathrm{~mm}-1$ (c) on bending angle of PAA/Fibrin hydrogel in cell culture medium, (d) Effect of Electrical field on responsive bending of $\mathrm{pSMC}$ seeded hydrogel, (e) Microscopic images of pSMCs seeded PAA/Fibrin hydrogel in static and stimulated conditions by alternative electrical field of $0.06 \mathrm{~V} \mathrm{~mm}-1$. Bending angle was measured as deviation of hydrogel middle line (red lines) from the state condition (yellow lines) in each 60 seconds before the polarization changes. The scale bars represent $2 \mathrm{~mm}$ in all the images. 

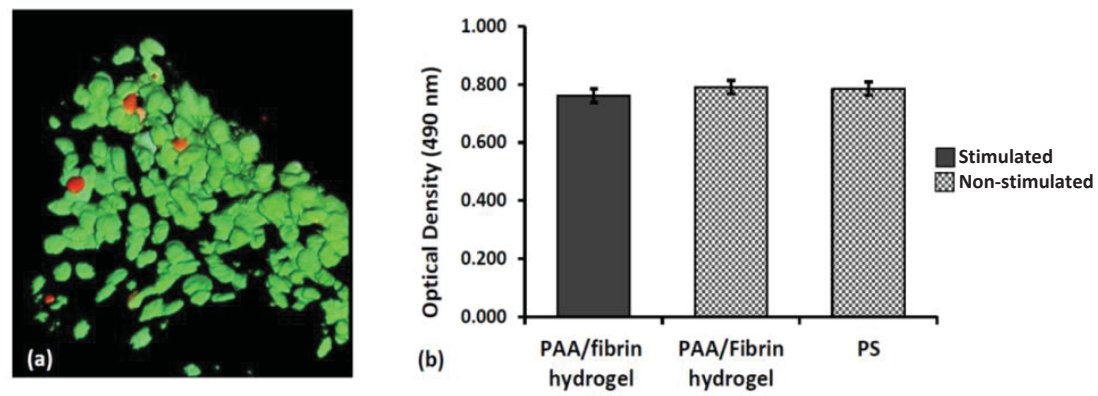

Fig. 6. (a) 2PLSM image of pSMCs seeded PAA/Fibrin stained with SYTO-13 (green) -PI (red) indicating alive and dead cells respectively after 24 hrs culturing. (b) MTS assay of pSMCs seeded PAA/Fibrin hydrogel, with and without electrical stimulation. PS:

control pSMCs culture on polystyrene plate. Differences are not significant.

\section{Cell distribution within the hydrogel}

Infiltration and density of pSMCs throughout the hydrogel was determined 1 week after seeding. Hydrogels were divided into 3 equal zones, tip, middle and root of the sample to evaluate the vertical distribution of cell density. There was a dense population of cells located in the first two layers close to the top seeded surface in the non-stimulated samples through the all parts of the sample (average density of 9,000 cell/mm3 in the first layer). This commonly observed distribution has been deemed inappropriate for homogeneous tissue formation [30, 44-46]. In contrast, in samples subjected to electrical field, cells penetrated down to $700 \mu \mathrm{m}$ in all the zones analyzed (Fig. 7a,b) with a fairly homogeneous distribution of cells over the depth of the structure. Differences in cell distribution between the zones were not significant.

3D images of the samples with and without stimulation show dispersion of cells through the $Z$ direction. The depth of cell infiltration into the samples was color coded, from red at the surface to dark pink at $700 \mu \mathrm{m}$ depth (Fig. 7ce). We considered that this appreciable improvement could be the consequence either of the induced cyclic mechanical changes in the gel or a direct effect of the electrical field on SMCs migration. In order to specify the 
driving force affecting cell behavior, SMCs L-type calcium channels were blocked by adding verapamil during stimulation. [26, 27].
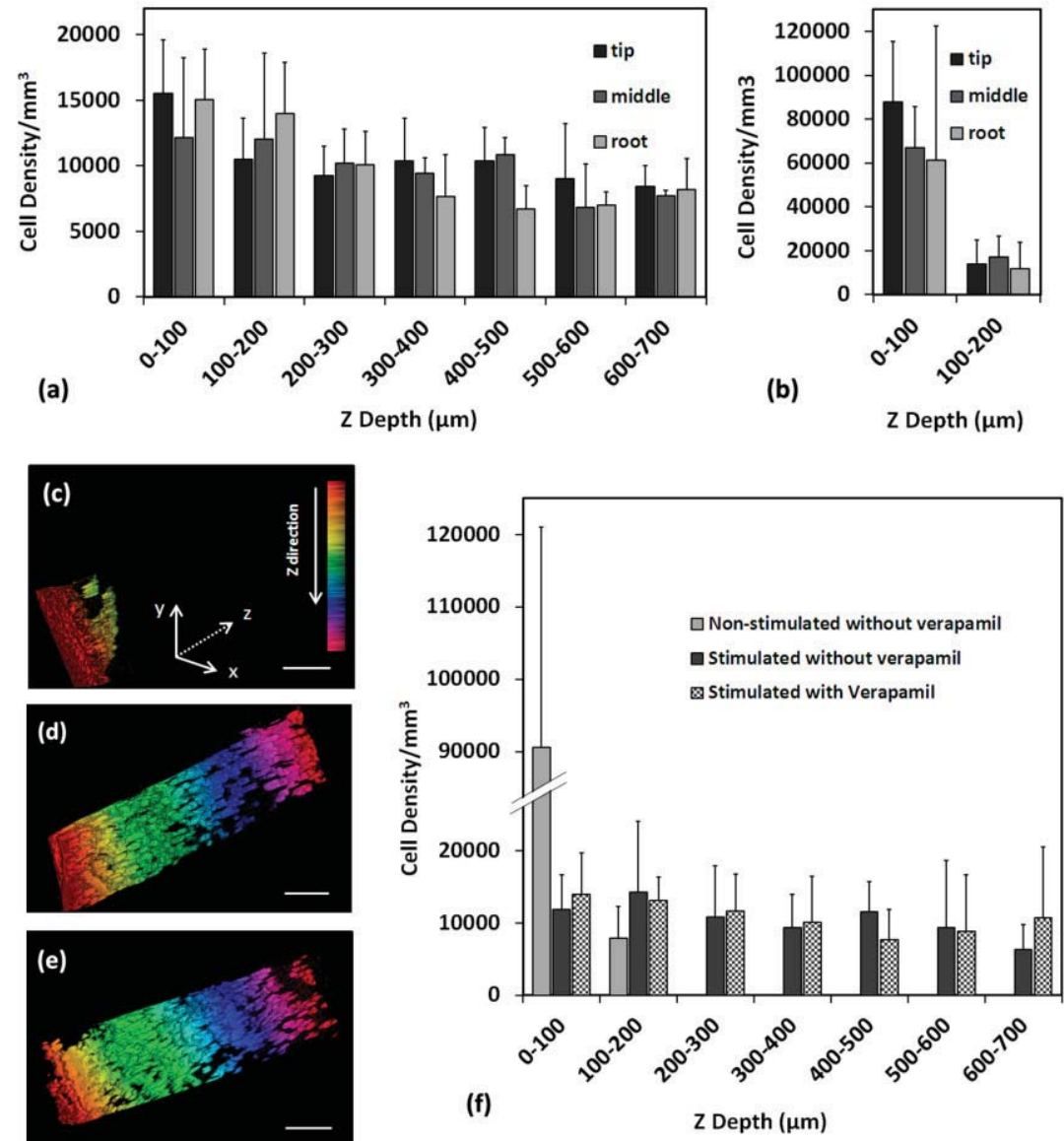

Fig .7. Cell distribution in the hydrogel samples. Panel a and b show distribution of pSMCs in seeded PAA/Fibrin hydrogels under stimulated condition by electrical field (alternative $0.06 \mathrm{~V} \mathrm{~mm}$ - 1 for $2 \mathrm{hrs}$ ) (a) and non-stimulated condition (b) in the long axis of the gel with a distinction in tip, middle and root region. Three-dimensional $Z$ coded 2PLSM images of pSMCs nucleus seeded in PAA/Fibrin hydrogels show distribution of cells throughout structure. (c) No electrical field was applied (d) electrical stimulation ( 2 hrs of alternating $0.06 \mathrm{~V} \mathrm{~mm}-1$ ) without verapamil treatment and (e) with electrical stimulation and verapamil treatment. (f) Total cell distribution in seeded PAA/Fibrin hydrogels in non-stimulated and stimulated conditions, with and without verapamil treatment, after 1 week of culturing. The scale bars represent $100 \mu \mathrm{m}$ in all the images. 
The effective concentration of verapamil was determined by evaluating electrical stimulus-induced contraction of SMCs cultured on fibronectin coated glass covers at different verapamil concentrations (data not shown). Because verapamil had negligible effect on the distribution of cells over the depth of the structure $(F=0.063 ; p=0.33$ and 0.80 for depth effect and verapamil-depth interaction, respectively. Fig. $7 \mathrm{~F}$ ), we conclude that the pulsatile mechanical stress of the gel is likely the major cause of SMCs distribution after seeding and not direct activation of SMCs by electrical stimulation. Applying low voltage $\left(0.06 \mathrm{~V} \mathrm{~mm}^{-1}\right)$ to an acrylic acid based hydrogel with free carboxylic groups is a non-invasive and tunable method to provide mechanical stress to the scaffold.

The mechanism for distribution of cells is still not clear. Mechanical stress could be one of the major factors leading to SMCs migration. Short mechanical stress-induction of cells has been shown to enhance SMC migration related to increased calcium-dependent protein kinases and Rhoactivated protein kinase activity $[47,48]$. In our setting, we showed that blocking calcium channels by adding verapamil did not change the distribution of cells throughout the hydrogel, which suggest SMC migration in our setting is calcium independent and likely not related to active contraction and relaxation of the cells. Further experiments are required to unravel the exact mechanism of transmigration through the electrically stimulated gel.

\section{Cell alignment}

Smooth muscle cells are highly aligned in helical patterns mostly circumferential and perpendicular to the long axis of the blood vessel. To ascertain the effect of electrical stimulation on SMC orientation and alignment we performed $a$-smooth muscle actin staining of stress fibers. Using 2PLSM, we observed actin filaments that were randomly oriented (Fig. 8a-d). Stimulation aligned the stress fibers along the width of the hydrogel (Fig. 8eh).

To quantify the alignment of cells and compare the effect of different conditioning on cell behavior, the angle of each actin fiber was measured according to a fixed reference line. Variation between fiber angles in stimulated samples with electrical field was at least 4 times less than in nonstimulated samples ( $n=4$ per group; K-S Test, $p<0.05$; Fig. 8i). Blocking L-type calcium channels by verapamil had no effect on probability distribution of 
fiber angles in unstimulated constructs $(p=0.14)$ or stimulated constructs $(p=0.18)$ and still allowed significant alignment of cells by stimulation compared with static condition $(p<0.05)$.

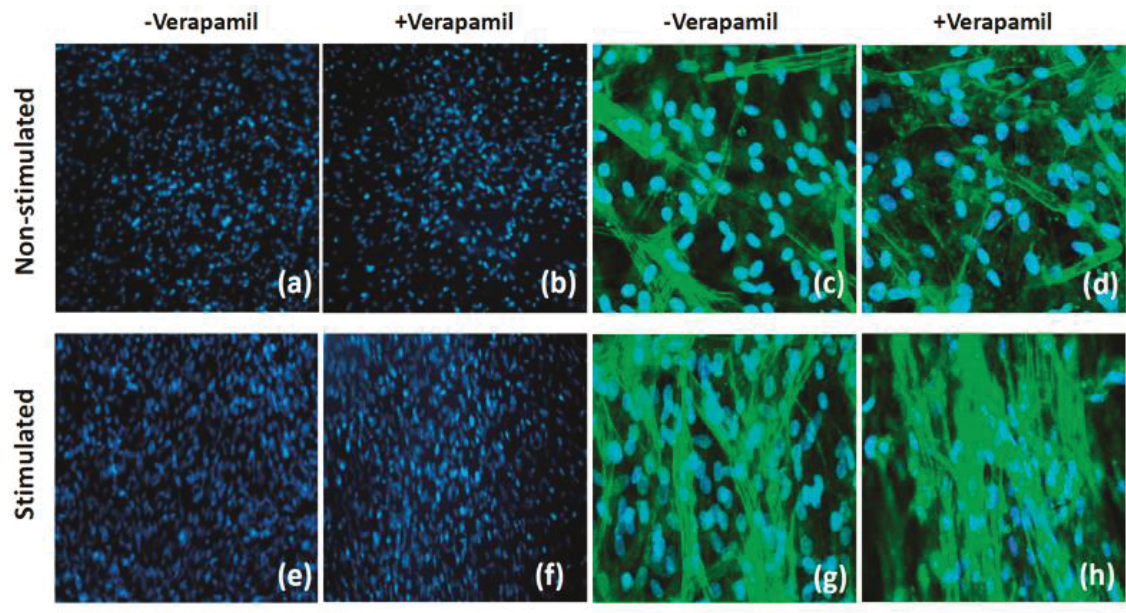

(i)

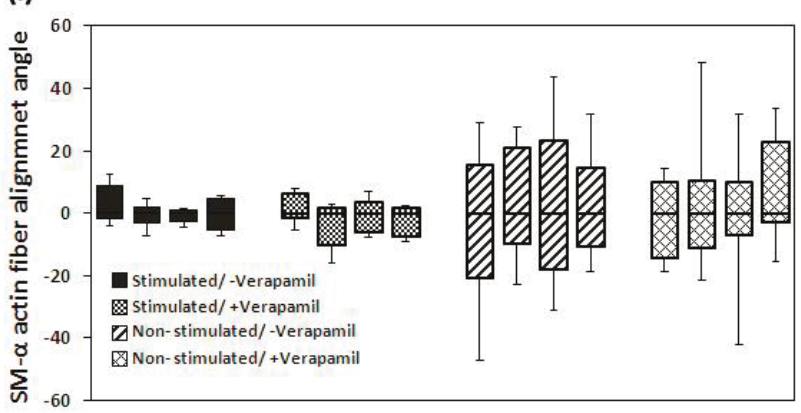

(j)

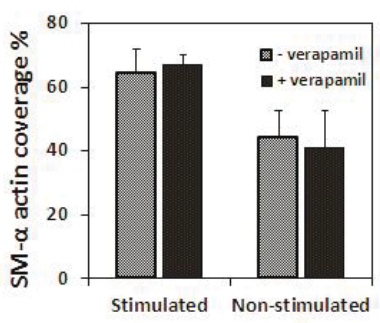

Fig. 8. 2 PLSM images of DAPI and DAPI/Alpha Smooth muscle actin filament alignment of pSMCs seeded PAA/Fibrin hydrogels after one week of culturing, in control samples in static condition with no L-type calcium channels blockade (verapamil) $(\mathrm{a}, \mathrm{c})$, with verapamil $(\mathrm{b}, \mathrm{d})$, and in stimulated samples exposed to alternative electrical field ( $0.06 \mathrm{~V}$ mm-1 for $2 \mathrm{hrs}$ ) without verapamil $(\mathrm{e}, \mathrm{g})$ and with verapamil $(f, h)$. The box plot shows variation in $a$-SM-actin alignment angles (i). Coverage of $\alpha$-SM-actin filaments in the samples with and without verapamil was not different (j). Magnification of DAPI and DAPI/a-SMA staining images is 10x and 40x, respectively. 
In addition, quantification of a-SM actin in samples showed pSMCs express higher $a$-SM actin under stimulated condition compared with non-stimulated after 7 days of culturing ( $50 \%$ increase, $p<0.05$ ). Furthermore, there was no significant difference in the $\alpha$-SM actin expression when adding verapamil for each condition (Fig. 8j).

When cells were seeded in the PAA/Fibrin hydrogel, they preferably attached to the bioactive Fibrin part of the structure, which could act as a temporary extracellular matrix (ECM). Orientation of Fibrin fibers parallel to the direction of applied stimulus of electrical field could result in elongation and orientation of SMCs along the Fibrin fibers in the construct. However, as published by Houtchens et al. our data indicate that alignment could occur by both microtopography of the structure and mechanical stress on the cells [49]. On the basis of the results, we demonstrated that mechanical forces produced by employing responsive ionic groups in the scaffold affected cell behavior leading to even dispersion of cells within the structure. Clinically, this approach can be used to develop an easy and promising step for modifying implanted grafts "in-vivo". Uniform distribution of produced ECM deposition can be achieved by controlling and inducing desired cellular properties in the scaffold, which will facilitate appropriate integration into the recipient tissue [50].

\section{Conclusion}

In the present study, a three-dimensional semi-IPN electro-responsive hydrogel for vascular tissue engineered grafts application was developed by incorporation of biodegradable Fibrin into the network of an electro-sensitive anionic polymer (acrylic acid). The short stimulation ( 2 hrs in the first day of seeding) of cyclic mechanical changes of the seeded gel induced by alternating electrical currents provided better cell penetration and alignment in the tissue construct.This system could be used to improve culture and seeding conditions for manufacturing tissue engineered vascular grafts. The data obtained so far, suggest this approach as a smart system that can be used as a coating or in combination with other polymers to improve design, mechanical properties and function of cellular vascular graft, which can enhance cell migration as well as facilitate perfusion of cell culture medium into the scaffold by applying regular stimulation pattern. 


\section{Acknowledgment}

This research program of the BioMedical Materials institute is co-funded by the Dutch Ministry of economic affairs Agriculture and Innovation. The financial contribution of the Nederlandse Hartstichting is gratefully acknowledged. We would also thank Geertje Swennen for her technical support and Mohammad Ghomi Rostami for his instrumental assistance. 


\section{Supporting information}
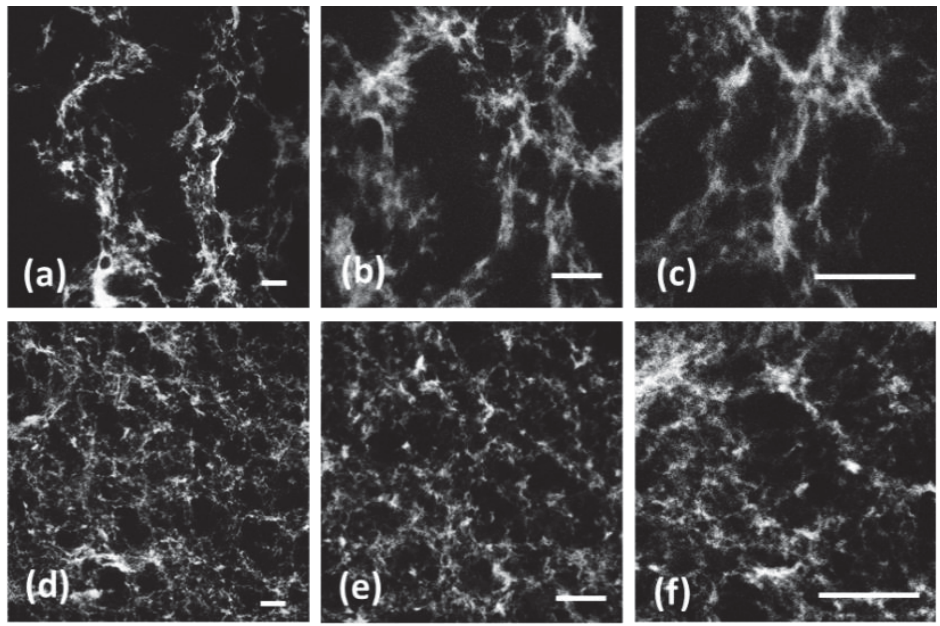

Fig. 1. Morphology of fibrin(ogen) dispersion in PAA/Fibrin hydrogel, stimulated by electrical field of alternating $0.06 \mathrm{~V} \mathrm{~mm}-1$ for $2 \mathrm{hrs}$ (a-c) and non-stimulated condition (d-f) at $\mathrm{pH}=6.5$, scale bars represents $100 \mu \mathrm{m}$ in all images. The direction of the electrical field was from top to bottom for each slide.

Video.1

This information is available free of charge via the Internet at: http://pubs.acs.org/doi/suppl/10.1021/bm300161r/suppl_file/bm300161r_si_001. avi 


\section{References}

1. Sarkar, S., G.Y. Lee, J.Y. Wong, and T.A. Desai, Development and characterization of a porous micro-patterned scaffold for vascular tissue engineering applications. Biomaterials, 2006. 27(27): p. 4775-82.

2. Aubin, H., J.W. Nichol, C.B. Hutson, H. Bae, A.L. Sieminski, D.M. Cropek, P. Akhyari, and A. Khademhosseini, Directed 3D cell alignment and elongation in microengineered hydrogels. Biomaterials, 2010. 31(27): p. 6941-6951.

3. Sarkar, S., M. Dadhania, P. Rourke, T.A. Desai, and J.Y. Wong, Vascular tissue engineering: microtextured scaffold templates to control organization of vascular smooth muscle cells and extracellular matrix. Acta Biomater, 2005. 1(1): p. 93-100.

4. Rose, F.R., L.A. Cyster, D.M. Grant, C.A. Scotchford, S.M. Howdle, and K.M.K.M. Shakesheff, In vitro assessment of cell penetration into porous hydroxyapatite scaffolds with a central aligned channel. Biomaterials, 2004. 25(24): p. 5507-5514.

5. Wang, C., L. Cen, S. Yin, Q. Liu, W. Liu, Y. Cao, and L. Cui, A small diameter elastic blood vessel wall prepared under pulsatile conditions from polyglycolic acid mesh and smooth muscle cells differentiated from adipose-derived stem cells. Biomaterials, 2010. 31(4): p. 621-30.

6. Liu, B., M.J. Qu, K.R. Qin, H. Li, Z.K. Li, B.R. Shen, and Z.L. Jiang, Role of cyclic strain frequency in regulating the alignment of vascular smooth muscle cells in vitro. Biophys J, 2008. 94(4): p. 1497-507.

7. Bai, H., C.D. McCaig, J.V. Forrester, and M. Zhao, DC electric fields induce distinct preangiogenic responses in microvascular and macrovascular cells. Arterioscler Thromb Vasc Biol, 2004. 24(7): p. 1234-9.

8. Rowlands, A.S. and J.J. Cooper-White, Directing phenotype of vascular smooth muscle cells using electrically stimulated conducting polymer. Biomaterials, 2008. 29(34): p. 4510-4520.

9. Li, X. and J. Kolega, Effects of direct current electric fields on cell migration and actin filament distribution in bovine vascular endothelial cells. J Vasc Res, 2002. 39(5): p. 391-404.

10. Mooney, K.Y.L.a.D.J., Hydrogels for Tissue Engineering. Chem. Rev., 2001. 101 (7): p. 1869-1880.

11. Kopecek, J., Hydrogel biomaterials: a smart future? Biomaterials, 2007. 28(34): p. 5185-92.

12. Drury, J.L. and D.J. Mooney, Hydrogels for tissue engineering: scaffold design variables and applications. Biomaterials, 2003. 24(24): p. 4337-51.

13. Slaughter, B.V., S.S. Khurshid, O.Z. Fisher, A. Khademhosseini, and N.A. Peppas, Hydrogels in regenerative medicine. Adv Mater, 2009. 21(32-33): p. 3307-29.

14. Qiu, Y. and K. Park, Environment-sensitive hydrogels for drug delivery. Adv Drug Deliv Rev, 2001. 53(3): p. 321-39.

15. Urban, F.L.M.W., Recent advances and challenges in designing stimuli-responsive polymers. Progress in Polymer Science, 2010. 35(1-2): p. 3-23.

16. Chaterji, S., I.K. Kwon, and K. Park, Smart Polymeric Gels: Redefining the Limits of Biomedical Devices. Prog Polym Sci, 2007. 32(8-9): p. 1083-1122.

17. Caldorera-Moore, M. and N.A. Peppas, Micro- and nanotechnologies for intelligent and responsive biomaterial-based medical systems. Adv Drug Deliv Rev, 2009. 61(15): p. 1391-401. 
8. Furth, M.E., A. Atala, and M.E. Van Dyke, Smart biomaterials design for tissue engineering and regenerative medicine. Biomaterials, 2007. 28(34): p. 5068-73.

19. TOYOICHI TANAKA, I.N., SHAO-TANG SUN and SHIZUE UENO-NISHIO, Collapse of Gels in an Electric Field. Science, 1982. 218(4571): p. 467-469.

20. Masao Doi, M.M., Yoshiharu Hirose, Deformation of ionic polymer gels by electric fields. Macromolecules, 1992. 25(20): p. 5504-5511.

21. Shiga, T. and T. Kurauchi, Deformation of polyelectrolyte gels under the influence of electric field. Journal of Applied Polymer Science, 1990. 39(11-12): p. 2305-2320.

22. Murdan, S., Electro-responsive drug delivery from hydrogels. J Control Release, 2003. 92(1-2): p. 1-17.

23. Shang, J., Z. Shao, and X. Chen, Electrical behavior of a natural polyelectrolyte hydrogel: chitosan/carboxymethylcellulose hydrogel. Biomacromolecules, 2008. 9(4): p. 1208-13.

24. Yin, L., L. Fei, F. Cui, C. Tang, and C. Yin, Superporous hydrogels containing poly(acrylic acid-co-acrylamide)/O-carboxymethyl chitosan interpenetrating polymer networks. Biomaterials, 2007. 28(6): p. 1258-1266.

25. Kaminer-Israeli, Y., J. Shapiro, S. Cohen, and A. Monsonego, Stromal cell-induced immune regulation in a transplantable lymphoid-like cell constructs. Biomaterials, 2010. 31(35): p. 9273-84.

26. Nawrath, H. and J.W. Wegener, Kinetics and state-dependent effects of verapamil on cardiac L-type calcium channels. Naunyn-Schmiedeberg's Archives of Pharmacology, 1996. 355(1): p. 79-86.

27. Itoh, Y.I.a.T., The roles of stored calcium in contractions of cat tracheal smooth muscle produced by electrical stimulation, acetylcholine and high $\mathrm{K}+\mathrm{Br} J$ Pharmacol, 1984. 83(3): p. 667-676.

28. Boerboom, R.A., K.N. Krahn, R.T.A. Megens, M.A.M.J. van Zandvoort, M. Merkx, and C.V.C. Bouten, High resolution imaging of collagen organisation and synthesis using a versatile collagen specific probe. Journal of Structural Biology, 2007. 159(3): p. 392-399.

29. Megens, R.T., S. Reitsma, P.H. Schiffers, R.H. Hilgers, J.G. De Mey, D.W. Slaaf, M.G. oude Egbrink, and M.A. van Zandvoort, Two-photon microscopy of vital murine elastic and muscular arteries. Combined structural and functional imaging with subcellular resolution. J Vasc Res, 2007. 44(2): p. 87-98.

30. Thevenot, P., A. Nair, J. Dey, J. Yang, and L. Tang, Method to analyze threedimensional cell distribution and infiltration in degradable scaffolds. Tissue Eng Part C Methods, 2008. 14(4): p. 319-31.

31. Peppas, N.A. and S.L. Wright, Solute Diffusion in Poly(vinyl alcohol)/Poly(acrylic acid) Interpenetrating Networks. Macromolecules, 1996. 29(27): p. 8798-8804.

32. Liu, Y. and M.B. Chan-Park, Hydrogel based on interpenetrating polymer networks of dextran and gelatin for vascular tissue engineering. Biomaterials, 2009. 30(2): p. 196207.

33. Bajpai, A.K. and M. Shrivastava, Swelling kinetics of a hydrogel of poly(ethylene glycol) and poly(acrylamide-co-styrene). Journal of Applied Polymer Science, 2002. 85(7): p. 1419-1428.

34. Bajpai, A.K. and A. Giri, Water sorption behaviour of highly swelling (carboxy methylcellulose-g-polyacrylamide) hydrogels and release of potassium nitrate as agrochemical. Carbohydrate Polymers, 2003. 53(3): p. 271-279. 
35. Yuk, S.H., S.H. Cho, and H.B. Lee, Electric Current-Sensitive Drug Delivery Systems Using Sodium Alginate/Polyacrylic Acid Composites. Pharmaceutical Research, 1992. 9(7): p. 955-957.

36. Jabbari, E., N. Wisniewski, and N.A. Peppas, Evidence of mucoadhesion by chain interpenetration at a poly (acrylic acid)/mucin interface using ATR-FTIR spectroscopy. Journal of Controlled Release, 1993. 26(2): p. 99-108.

37. Yang, Q., Y. Zhang, M. Liu, M. Ye, Y. Zhang, and S. Yao, Study of fibrinogen adsorption on hydroxyapatite and $\mathrm{TiO}_{2}$ surfaces by electrochemical piezoelectric quartz crystal impedance and FTIR-ATR spectroscopy. Analytica Chimica Acta, 2007. 597(1): p. 58-66.

38. Li, Q., J. Wu, Z. Tang, Y. Xiao, M. Huang, and J. Lin, Application of poly (acrylic acid-ggelatin)/polypyrrole gel electrolyte in flexible quasi-solid-state dye-sensitized solar cell. Electrochimica Acta, 2010. 55(8): p. 2777-2781.

39. Lin, J., Q. Tang, D. Hu, X. Sun, Q. Li, and J. Wu, Electric field sensitivity of conducting hydrogels with interpenetrating polymer network structure. Colloids and Surfaces A: Physicochemical and Engineering Aspects, 2009. 346(1-3): p. 177-183.

40. Fehér, J., G. Filipcsei, J. Szalma, and M. Zrínyi, Bending deformation of neutral polymer gels induced by electric fields. Colloids and Surfaces A: Physicochemical and Engineering Aspects, 2001. 183-185: p. 505-515.

41. Seon Jeong, K. and et al., Properties of smart hydrogels composed of polyacrylic acid/poly(vinyl sulfonic acid) responsive to external stimuli. Smart Materials and Structures, 2004.13(2): p. 317.

42. Kim, S.Y., H.S. Shin, Y.M. Lee, and C.N. Jeong, Properties of electroresponsive poly(vinyl alcohol)/poly (acrylic acid) IPN hydrogels under an electric stimulus. Journal of Applied Polymer Science, 1999. 73(9): p. 1675-1683.

43. Sun, S. and A.F.T. Mak, The dynamical response of a hydrogel fiber to electrochemical stimulation. Journal of Polymer Science Part B: Polymer Physics, 2001. 39(2): p. 236246.

44. Soletti, L., A. Nieponice, J. Guan, J.J. Stankus, W.R. Wagner, and D.A. Vorp, A seeding device for tissue engineered tubular structures. Biomaterials, 2006. 27(28): p. 4863-70.

45. Nieponice, A., L. Soletti, J. Guan, B.M. Deasy, J. Huard, W.R. Wagner, and D.A. Vorp, Development of a tissue-engineered vascular graft combining a biodegradable scaffold, muscle-derived stem cells and a rotational vacuum seeding technique. Biomaterials, 2008. 29(7): p. 825-33.

46. Weinand, C., J.W. Xu, G.M. Peretti, L.J. Bonassar, and T.J. Gill, Conditions affecting cell seeding onto three-dimensional scaffolds for cellular-based biodegradable implants. J Biomed Mater Res B Appl Biomater, 2009. 91(1): p. 80-7.

47. Li, C., F. Wernig, M. Leitges, Y. Hu, and Q. Xu, Mechanical stress-activated PKCdelta regulates smooth muscle cell migration. FASEB J, 2003.17(14): p. 2106-8.

48. Gerthoffer, W.T., Mechanisms of vascular smooth muscle cell migration. Circ Res, 2007. 100(5): p. 607-21.

49. Houtchens, G.R., M.D. Foster, T.A. Desai, E.F. Morgan, and J.Y. Wong, Combined effects of microtopography and cyclic strain on vascular smooth muscle cell orientation. J Biomech, 2008. 41(4): p. 762-9.

50. D. Wendt, S.S., M. Jakob, G.T. John, I. Martin, Uniform tissues engineered by seeding and culturing cells in $3 \mathrm{D}$ scaffolds under perfusion at defined oxygen tensions. Biorheology, 2006. 43(3-4): p. 481-488. 
Electro-sensitive Hydrogel Facilitates Cell Seeding and Alignment

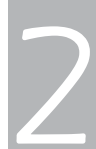




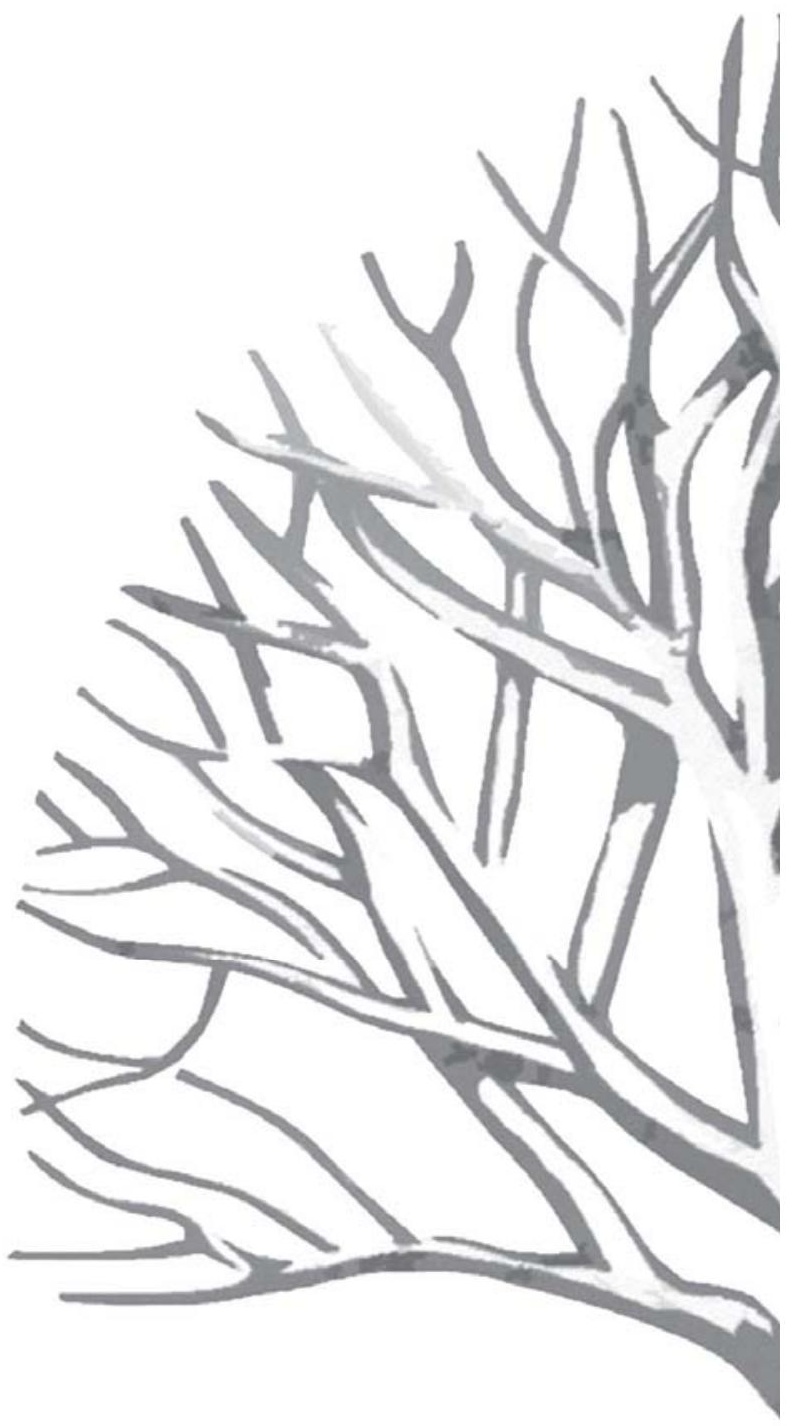




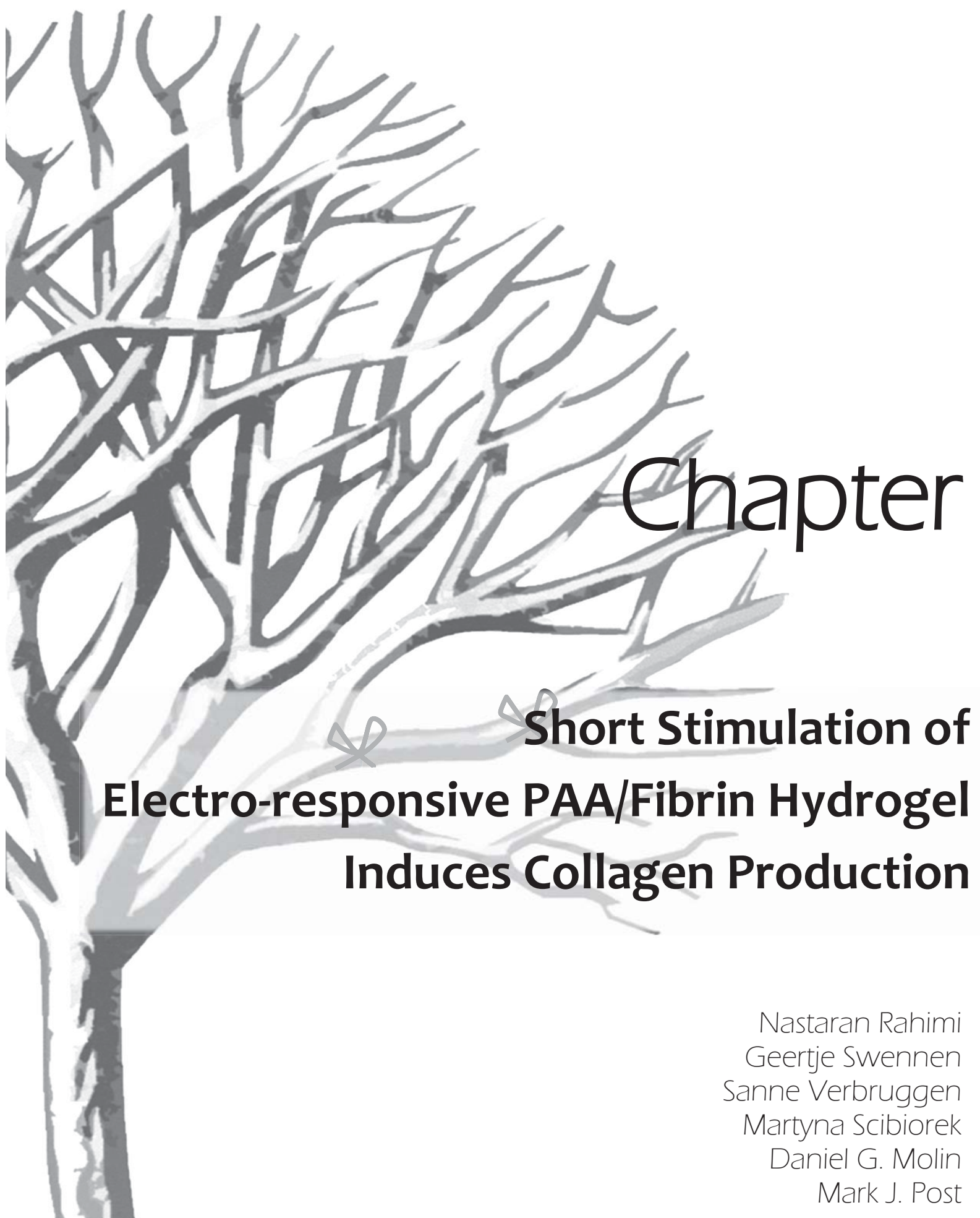

Tissue engineering 2014, 20 (9):703-13 


\section{Abstract}

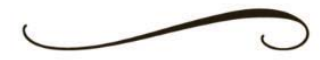

Acrylic acid/Fibrin hydrogel can mechanically stimulate cells when an external electrical field is applied, allowing them to migrate and align throughout the depth of the gel. The ability of electro-responsive Polyacrylic acid/Fibrin hydrogel to promote collagen production and remodeling has been investigated by 3D culturing and conditioning of smooth muscle cells (SMCs). SMCs seeded hydrogels were subjected to an alternating electrical field ( 0.06 $\mathrm{v} / \mathrm{mm}$ ) for 2 hours either for one, two or three times per wk during 4 wks of culturing. Fluorescent images of collagen structure and accumulation, assessed by CNA-35 probe, showed increased collagen content (>100-fold at $1 \mathrm{x}$ stimulation/wk) in the center of the hydrogels after 4 wks of culture. The increase in collagen production correlated with increasing extracellular matrix genes expression and resulted in significantly improved mechanical properties of the stimulated hydrogels. MMP2 activity was also significantly enhanced by stimulation, which probably has a role in reorganization of the collagen. Short stimulation period (2hrs) induced a favorable response in the cells and enhanced tissue formation and integrity of the scaffold by inducing collagen production. The presented set-up could be used for conditioning and improving the functionality of current tissue engineered vascular grafts. 


\section{Introduction}

Deposition of extracellular matrix (ECM) by cells in tissue-engineered (TE) constructs plays an important role in providing a long-term functional substrate to regulate cell-tissue interaction $[1,2]$. For replacement of mechanically active tissues such as blood vessel grafts, it is compulsory that TE constructs are sufficiently strong to withstand physiological forces such as blood pressure [3]. The medial layer of blood vessels consists primarily of smooth muscle cells (SMCs), collagen (mainly type I and III), elastin and proteoglycans that influence vessel function by providing appropriate stiffness, elasticity and compressibility [3]. Collagen is the most abundant ECM protein in vascular tissue and its structural arrangement is responsible for the tensile strength [4].

Environmental conditioning of seeded TE constructs can effectively influence ECM production and tissue formation. For example, mechanical stimuli such as cyclic strain have been shown to enhance collagen production in SMC seeded scaffolds [4-7].

Previously, we have reported on the preparation, optimization and characterization of an electro-sensitive Polyacrylic acid/Fibrin hydrogel, showing that short electrical stimulation resulted in high cell alignment and distribution [8]. Polyacrylic acid is a known biocompatible anionic polymer. Acrylic hydrogels and their derivatives polymers have been already used as FDA approved basic component of dental and bone cement implants and intraocular lenses $[9,10]$. Also, based on the high charge density of Polyacrylic acid they have been used as good candidate for mucoadhesives [11].

By applying an electrical field to the hydrogel, carboxylic groups of Polyacrylic acid network dissociate into mobile positive $\mathrm{H}^{+}$and negatively charged - $\mathrm{COO}$ groups. The attraction of ions towards their opposite electrodes induces swelling of the hydrogel at the cathode side and shrinkage at the anode side. Thereby the Polyacrylic acid hydrogel is pulled towards the positive electrode anode (Fig. 1). By changing the direction of electrical field, rhythmic deformation (bending) of the hydrogel is achieved, which regulates cell behavior by inducing alignment of Fibrin fibers, cells and smooth muscle alphaactin filaments [8].

In this study, we investigate the long-term effect of different regimes of electrical stimulation on cell density and collagen production of SMCs seeded electro-sensitive hydrogels. Synthesis, accumulation and organization of collagen play an important role in regulating tissue growth and providing a 
mechanically functional graft with appropriate strength. Collagen production was visualized by staining the samples with a CNA-35 probe, a high affinity collagen binding protein that binds to the most abundant fibrillar collagens I, III and IV present in the native vessel wall [12, 13]. The effect of electrical stimulation on collagen remodeling was assessed by measuring the activity of two major Matrix Metalloproteinases (MMPs) produced by SMCs (i.e. MMP2 and -9) $[14,15]$.

\section{Materials and methods}

\section{Preparation of Polyacrylic acid/Fibrin hydrogels}

Polyacrylic acid (PAA)/Fibrin hydrogels were synthesized as previously described [8]. Briefly, Acrylic acid (AA, purity 99.9\%, Merck, Germany) was cross-linked and polymerized in presence of Fibrin $(20 \mathrm{mg} / \mathrm{mL}$ Fibrinogen mixed by $20 \mathrm{IU} / \mathrm{mL}$ thrombin, bovine plasma, Sigma Aldrich) by adding methylenebisacrylamide (MBAA, 2 wt \% monomer, Sigma Aldrich), ammonium persulfate (APS, 0.2 mol\% of the AA, Sigma Aldrich) and tetramethylenediamide (0.4 mol \% of AA, TMEDA, Sigma Aldrich) as cross-linker, initiator and accelerator, respectively. All components were dissolved in deionized water (1:1 weight ratio of acrylic acid to Fibrin), molded between two plates with 1 $\mathrm{mm}$ spacer and heated at $80{ }^{\circ} \mathrm{C}$ for $3 \mathrm{~h}$. All chemicals used in hydrogel preparation were of molecular cell biology grade without further purif cation.

\section{Hydrogel cell seeding}

Lyophilized hydrogels were prepared by freeze-drying and UV sterilization as previously described [8]. Hydrogel strips $(15 \mathrm{~mm} \times 4 \mathrm{~mm} \times 1 \mathrm{~mm})$ were fixed at one end by tissue glue (Vetbond Tissue Adhesive, USA) in a 4-well plate. Primary porcine smooth muscle cells (pSMCs) were isolated from pig aorta, cultured and expanded until passage 8-10, on Fibronectin coated flasks containing smooth muscle basal medium (SmBM, Lonza) supplemented with $5 \%$ fetal bovine serum (FBS), human epidermal growth factor, human fibroblast growth factor-B, insulin, and gentamicin. Cells were suspended in $100 \mu \mathrm{L}$ with a final cell density of $1.5 \times 10^{6}$ cells/sample and seeded on the surface of the hydrogels. Samples were incubated at $37^{\circ} \mathrm{C}$ with $5 \% \mathrm{CO}_{2}$ (Fig. 1a). 


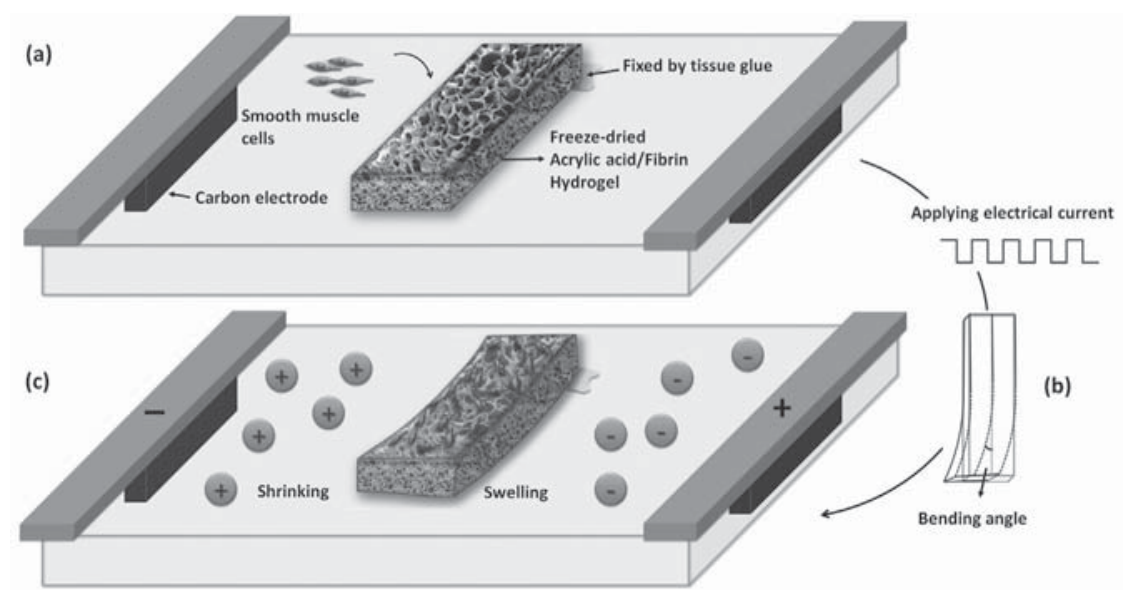

Fig. 1. Graphic overview of the hydrogel mounting-fixing and cellular seeding in the culture plate equipped with carbon electrodes (a). Electrical current is applied to the cells, 4 hrs after seeding. Hydrogel swells at the side of the positive electrode side and shrinks at the negative one due to attraction and migration of negative/positive ions toward opposite electrodes (b,c). Changes in the direction of the current $(0.0167 \mathrm{~Hz})$

cause reversible bending of the hydrogel.

\section{Stimulation of cell-seeded hydrogel}

To determine the optimal stimulation regime, different patterns of electrical field were applied to the pSMCs seeded hydrogels. We studied the effect of 4 different stimulation conditionings, including a single stimulation at the first day of seeding, followed by one, two or three stimulations per wk. For each stimulation, the seeded hydrogel which had been fixed at one end in the 4 well plate that was equipped with carbon electrodes, was exposed for 2 hrs to an alternating electrical field of $0.06 \mathrm{~V} / \mathrm{mm}$. An electrical power supply (Bio-Rad) connected to an in house developed programmable switch, provided the electrical field with changing current direction every 60 seconds to induce reversible swelling/deswelling at both sides of the gel [8] (Fig. 1 b,c). To enable initial cell attachment and infiltration, samples were exposed to their first electrical stimulation 4 hrs after cell seeding. To block SMC contraction by electrical stimulation and investigate only the effect of produced mechanical force, Verapamil (10 $\mu \mathrm{M}$, Sigma Aldrich, The Netherlands) was added to the culture medium. Smooth muscle cells in Fibrin gels without the electro- 
responsive PAA component were used to evaluate the effect of electrical field only as Fibrin itself does not undergo any conformational change in an electrical field. Non-stimulated cultured samples served as controls for all the experiments.

\section{Microscopy}

The stained samples were scanned and imaged with a two-photon laser scanning microscope (2PLSM, Biorad, Great Britain) coupled to a Nikon E60oFN microscope (Nikon, Tokyo, Japan) with Ti-Sapphire laser excitation source of 140-fs-pulsed (Spectra Physics Tsunami, Mountain View, CA) tuned and modelocked at $800 \mathrm{~nm}$ (for visualization of $\mathrm{f}$ uorescent probes) [16, 17]. To determine the collagen production, pSMCs seeded hydrogels were washed with Phosphate Buffer Solution (PBS 1x, Lonza) and fixed in paraformaldehyde $2 \%$ for 20 minutes at $4^{\circ} \mathrm{C}$. Samples were then washed thrice in PBS $1 \times$ and incubated with the CNA35 conjugated Alexa Fluor ${ }^{\circledR} 647$ collagen probe (CNA35 in house synthesis, diluted 1:10 in HBSS, Alexa Fluor Lifetechnologies,) for 60 minutes, followed by DAPI for cell nucleus staining (diluted 1:1,000 in PBS) for 20 minutes at room temperature [17].

To scan the samples by 2 PLSM, hydrogels were put in a plate, filled by liquid $2 \%$ Agarose gel, and cooled down in room temperature to solidify and fix the sample. A small amount of deionized water (MiliQ) was added on top of the gels to enable scanning with a 40x water-dipping objective (2.0 $\mathrm{mm}$ working distance, numerical aperture (NA) 0.8, Nikon). Hydrogel surface images were collected in the $x-y$ plane. To scan the hydrogels center section, $z$ stack images were recorded from the depth center (approximately $400 \mu \mathrm{m}$ from top seeded surface) in three different parts of the sample and continued through the thickness of the structure (z direction) with interval z-steps of 2-4 mm for approximately $300 \mu \mathrm{m}$.

ImageJ (National Institutes of Health, Bethesda, MD) software was used to quantify and analyze the collected images. Cell density was calculated by counting DAPI stained cell nuclei of each image ( $x-y$ plane) using Nucleus Counter Plug-in. Collagen was quantified by converting the images presenting CNA-35 stained areas to 8-bit images and measured the adjusted threshold area in each section by using the Area Calculator plug-in. Total cell density and collagen area in the (top-seeded) surface were calculated on more than 6 spots $(x-y)$ for each sample $(n=3)$. For the center section of the hydrogels, an average value of at least 18 images for each condition, including 6 images in the $z$ - 
direction from three spots $(x-y)$ was used. 3-D images of stained hydrogels were built up and rendered by using the VolumeJ builder plug-in (v.1.7a, Michael Abramoff).

\section{Reverse transcriptase-polymerase chain reaction}

Protein gene expression in the TE constructs was measured by performing Real time reverse transcription polymerase chain reaction (RT-PCR) by using a BIORAD MiQ5 (Biorad, Veenendaal, The Netherlands) with Quanta reagents (VWR international BV, Amsterdam, The Netherlands).

Data analysis was performed by using Biogazelle Qbase (Biogazelle, Gent, Belgium). pSMCs seeded hydrogels were cultured and RNA was collected after the last stimulation at 4 th wks. To extract total RNA, samples were freezedried, dissolved by RNeasy lysis buffer (RLT, Qiagen) and mechanically destroyed by Ultraturax homogenizer to disrupt tissue and cell membranes. A total of 100 ng RNA per sample was subjected to reverse transcription (RT). qPCR was performed by two steps Syber Green kit protocol from Quanta with a 5 minute $45^{\circ} \mathrm{C}$ incubation pre-run step and $40 \mathrm{PCR}$ cycles with $59^{\circ} \mathrm{C}$ annealing temperature using the primers as listed in Table 1. qPCR reactions were run on a BioRad MiQ Real Time PCR detection System (BioRad, Veenendaal, the Netherlands). Expression levels of Col1a1, Col1a2, Col3a1 and ELN were normalized to expression of Beta-Actin and HPRT, which stayed constant during stimulation and control culture conditions. The samples collected before stimulation were considered as controls and used to normalize the data (set at 1).

Table1. Forward and reverse primers used for RT-PCR of smooth muscle cell seeded PAA/Fibrin hydrogels

\begin{tabular}{c|cc}
\hline Gene & FWD & REV \\
\hline Col $1 \alpha 1$ & AAGAAGAAGGCCAACAAGG & CATCGCACAACACATTGC \\
Col $1 \alpha 2$ & GAGATGGTGATGATGGTATCC & ACTGAGCAGCAAAGTTCC \\
Col3 $\alpha 1$ & GAGCTTCCCAGAACATCACA & CACCTTCATTTGATCCCATC \\
Elastin & GTGGAAGCTTTTCTGGAAT & GCAGTTTCCCAGTGCTGTAG \\
\hline
\end{tabular}




\section{Hydrogel bending and compaction}

pSMCs seeded hydrogels were either not stimulated (controls) or exposed to stimulation at the first day of seeding and, subsequently, one or three stimulations per wk, each for 2 hrs. All samples were cultured for 4 wks in total. Digital images of hydrogels were taken every other day by a microscope with a built-in camera (EVOS, AMG, USA). Hydrogel width was measured from all images using ImageJ ( $\mathrm{n}=3$ for each condition). The compaction ratio was calculated as follows: $\left(W_{t}-W_{i}\right) / W_{i} \times 100 \%$, where $W_{i}$ and $W_{t}$ represent the width of the hydrogel at initial condition and time $t$, respectively. Reversible deformation of the hydrogels was recorded during their exposure to electrical field and measured as a deviation of the hydrogel strip from a fixed middle line along the hydrogel as a reference and defined as bending angle (Fig. 1).

\section{Uniaxial tensile test}

Mechanical properties of pSMC seeded PAA/Fibrin hydrogels were determined by a uniaxial testing machine (Tainstruments Q800) with the use of an 18-N load cell at a cross-head speed of $1 \mathrm{~mm} / \mathrm{min}$. All the samples were approximately $3 \mathrm{~mm} \times 10 \mathrm{~mm} \times 1 \mathrm{~mm}$ (width $\times$ length $\times$ thickness) and had been cultured for $4 \mathrm{wks}$, either in stimulated or static condition. Samples were mechanically measured in their swollen hydrated state. At least 4 samples were tested for each condition.

\section{Gelatin zymography}

Activity of matrix metalloproteinase (MMP) was analyzed in conditioned medium obtained from cultured $\mathrm{pSMC}$ seeded PAA/Fibrin hydrogels or $\mathrm{pSMC}$ seeded Fibrin gels. The media of samples 2 hrs before the stimulation, during the stimulation ( $2 \mathrm{hrs}$ ) and in the non-stimulated control condition were collected, stored at $-20^{\circ} \mathrm{C}$ and centrifuged prior to the measurement. MMP activity was assessed by Gelatin Zymography as MMP-2 and MMP-9 are capable of degrading Gelatin. Zymography was performed by using $10 \%$ Acrylamide/Gelatin gel (Novex, Invitrogen). Equal volumes of media were loaded into the Zymogram gels and run at $125 \mathrm{~V}$ for $90 \mathrm{~min}$. To reactivate MMP activity, gels were washed in re-naturing buffer and subsequently, incubated in 
developing buffer overnight. Gels were stained with SimplyBlue ${ }^{\mathrm{TM}}$ Safestain (Invitrogen) and rinsed in deionized water before detection with a BioRad camera and quantification by Quantity One ${ }^{\circledR}$ Analysis software (BioRad Laboratories).

\section{Statistical analysis}

All quantitative experiments were performed in triplicate or more, and data were expressed as means + standard deviation. To compare groups' multiple means with one or two parameters, statistical analysis of the results was performed either by t-test, one-way ANOVA (Analysis of Variance) or two-way ANOVA (repeated measures) with Bonferroni post hoc analysis (Graphpad prism 4, Graphpad Prism Software Inc., San Diego, USA). The correlation of variables between two groups was examined by calculating the Pearson correlation coefficient (Graphpad prism 4). P values $<0.05$ were considered to be significant in all the analysis.

\section{Results}

\section{Cell density and collagen production after 4 wks of culture}

Hydrogels were stained with DAPI/CNA35 probes and scanned by 2PLSM at the end of $4 \mathrm{wks}$ culture to check the cell density and collagen-matrix production in the surface and center section of the samples. Images were quantified by using ImageJ software.

Confirming our earlier results with a short culture period, the cells were aligned and homogenously distributed in the stimulated samples compared to random orientation in the control samples after 4 wks of culturing (Fig. 2a-d). Stimulated samples revealed profound fibrillar network formation of collagen compared to random spot deposition of collagen in non-stimulated hydrogels after 4 wks of culture. We observed similar cell density at the surface of hydrogels in the non-stimulated and stimulated conditions (Fig. 2e), however collagen production was significantly enhanced at the surface of the stimulated samples compared with controls (Fig. 2f; 3.7 fold; $p<0.0001$ ). In the center section of the stimulated samples cell density was more than 6 times increased compared to non-stimulated ones (Fig. 2h). The amount of collagen in the center of stimulated hydrogels was 85 -fold increased compared to 
controls (Fig. 2i; $\mathrm{p}<0.001$ ) as a result of higher collagen production per cell (Fig. 2j, 8 fold, $p<0.01$ ) in the stimulated samples.

Applying mechanical load to the cells could augment collagen production through either increasing proliferation or increasing the procollagen synthesis per cell [18]. Improved distribution of the cells accross the depth of the stimulated hydrogels accompanied by enhanced capacity of cells to produce more collagen resulted in much higher collagen accumulation in the stimulated samples after 4 wks.

\section{Stimulation frequency affects collagen production after 4 wks of culture}

To evaluate the effect of stimulation frequency on pSMCs collagen and tissue production in the samples, different stimulation patterns were applied to seeded hydrogels during 4 wks of culturing. Hydrogels were first all exposed to electrical stimulation on the seeding day, and were followed by 1,2 or 3 stimulations (each for $0.06 \mathrm{~V} / \mathrm{mm}, 0.0167 \mathrm{~Hz}, 2 \mathrm{hrs}$ ) per wk for the rest of the 4 wk culture. Non-stimulated samples served as controls. Hydrogels were stained with DAPI/CNA35 and scanned by 2PLSM at the end of 4-wk culture (Fig. 3 a-I). Similar to the short culture time ( $1 \mathrm{wk}$ ) with only one stimulation on the day of seeding, continued intermittent stimulation up to 2 times per wk, resulted in more condense collagen fiber formation compared to non-stimulated controls after 4 wks (Fig. 3a-i). These samples showed enhancement in collagen production both at the surface and in the center while at 3 stimulations per wk collagen was disorganized, showing deposition mainly as cytoplasmatic spots without fibrillar appearance (Fig. $3 \mathrm{j}-\mathrm{I}$ ).

Although total cell density in the surface was not affected by stimulation, a 7fold increase in cell density in the center was observed (Fig. 3m,n). The collagen production at the surface was enhanced by approximately 3 - and 2.5fold in the 1 and 2 stimulations per wk compared to non-stimulated control, respectively (Fig. 30). Notably, in the center of the gel, the increase in collagen production was strongly augmented to 120 - and 100-folds respectively, compared to the non-stimulated control condition (Fig. 3p). Although the 3 stimulations per wk showed no significant difference in morphology and collagen amount at the surface of hydrogel compared with controls (Fig. 3 a,j), in the hydrogel center section the collagen amount was significantly higher than controls (Fig. 3 p, 46-fold, $p<0.01$ ). 

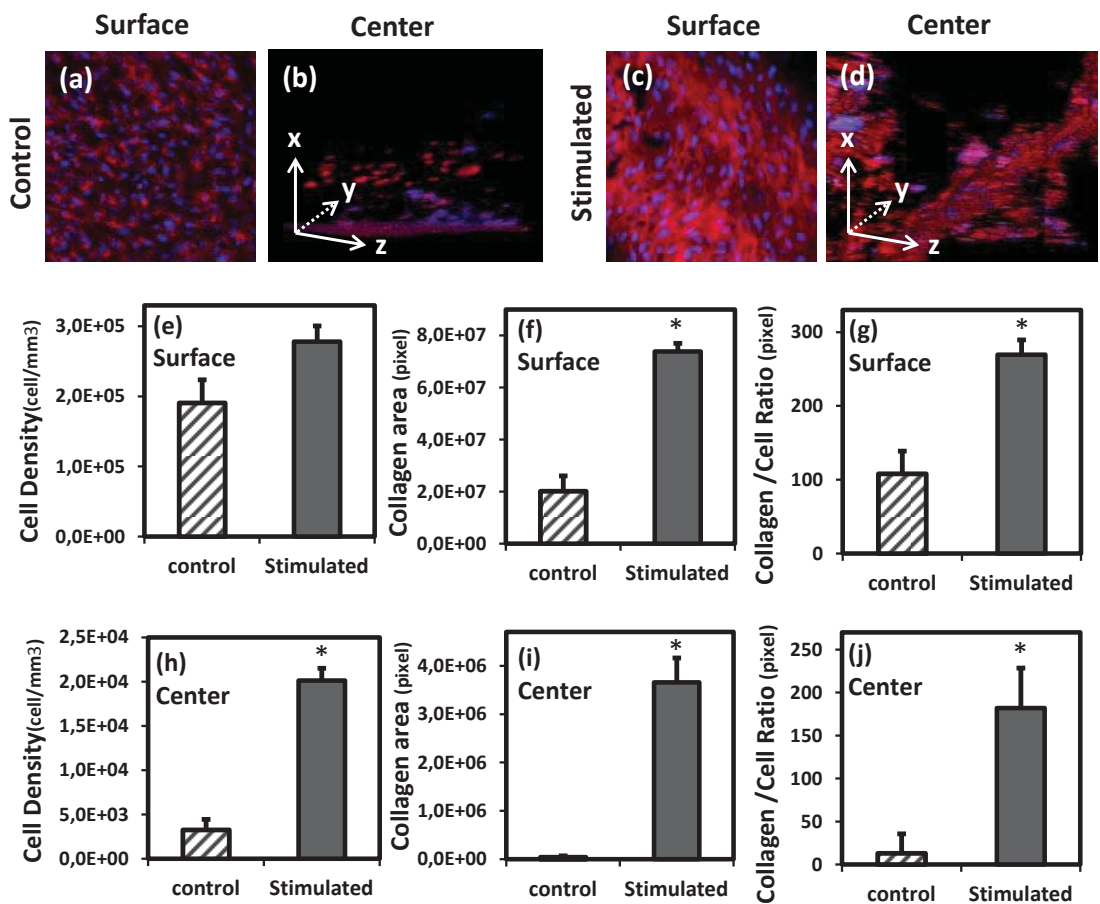

Fig. 2. Two photon laser scanning microsopy images of SMCs (DAPI, blue) and Collagen organizationn (CNA-35, red) from the surface and center cross-section ( $z$ direction) of the non-stimulated (control) (a-b) and stimulated constructs seeded with pSMCs of one stimulation in the first day $(c, d)$ after 4 wks of culture. Total cellular density $(e, h)$, collagen area $(f, i)$ and collagen production per cell $(g, j)$ quantified at the surface and in the center cross section of hydrogels. Asterisks indicate $P$ value $<0.05$, stimulated versus control.

Stimulated samples showed no significant difference in the collagen production per cell in the surface and center section, while in control hydrogels, there was a significant 5 -fold decrease in the hydrogel center compared to the surface (Fig. 3q,r; p<0.05). Considering fibrillar network of collagen, collagen content and collagen production per cell, the optimal protocol appears to be 1 electrical stimulation per wk.

Therefore, we also evaluated the effect of stimulation on the expression of extracellular matrix (ECM) genes including Collagen1a1 (Col1a1), Collagen1a2 (Col1a2), Collagenza1 (Col3a1) and Elastin in the samples stimulated once per wk. RT-PCR analysis was performed on PSMC RNA isolated from the hydrogels to estimate their expression levels for the 4-wk culture (Fig. 3s). RNA was 
collected from samples before and after stimulation, The samples collected before stimulation were considered controls and used to normalize the data. Stimulation led to higher expression of the matrix genes Col1a1, Col1a2, Col3a1 and Elastin by $54 \%, 111 \%, 72 \%$ and $185 \%$, respectively, compared with the priorstimulation condition. The obtained data corresponded with the higher production of collagen at 4 wks upon stimulation represented by 2PLSM images and confirmed the fibrillar collagen as it mainly consisted of collagen type I and III [19].

\section{Hydrogel compaction and its electro-responsive bending after 4 wks of culture}

pSMC seeded PAA/Fibrin hydrogels subjected to alternating electrical field once at the day of seeding only or additionally one to three times per wk during $4 \mathrm{wks}$ of culturing were evaluated for their degree of bending and compaction. During the stimulation, samples were evaluated by collecting light microscopy images for bending and every other subsequent day for compaction (Fig. 4).

Just two days after seeding, a significant increase in the compaction ratio (>6fold) for stimulated samples compared with control was noted (Fig. 4b). No significant difference was found between the compaction of samples stimulated with different regimes. Indeed hydrogel stimulated once after cell seeding followed the same path as the samples stimulated 3 times per wk for 4 wks. After the initial increase in stimulated samples, compaction of the hydrogel increased in the stimulated and control gels during 4 wks follow up, with a similar trend. The observation suggests that the reorganization of the Acrylic acid/Fibrin network, which occurs during the first stimulation, plays an important role in the compaction. We previously showed high alignment of Fibrin fibers in the direction of the electrical field after a one time stimulation ( $0.06 \mathrm{~V} / \mathrm{mm}, 0.0167 \mathrm{~Hz}, 2 \mathrm{hrs}$ ) in PAA/Fibrin hydrogels [8]. The bending angles of pSMCs seeded hydrogels were also recorded during each stimulation. In the samples stimulated thrice per wk, the bending angle significantly increased over time starting with 1.5 degrees on the day of seeding and reaching a max of 2.7 degrees (+/- 4\%) at 25 days (Fig. 4C; $p<0.01$ ). 

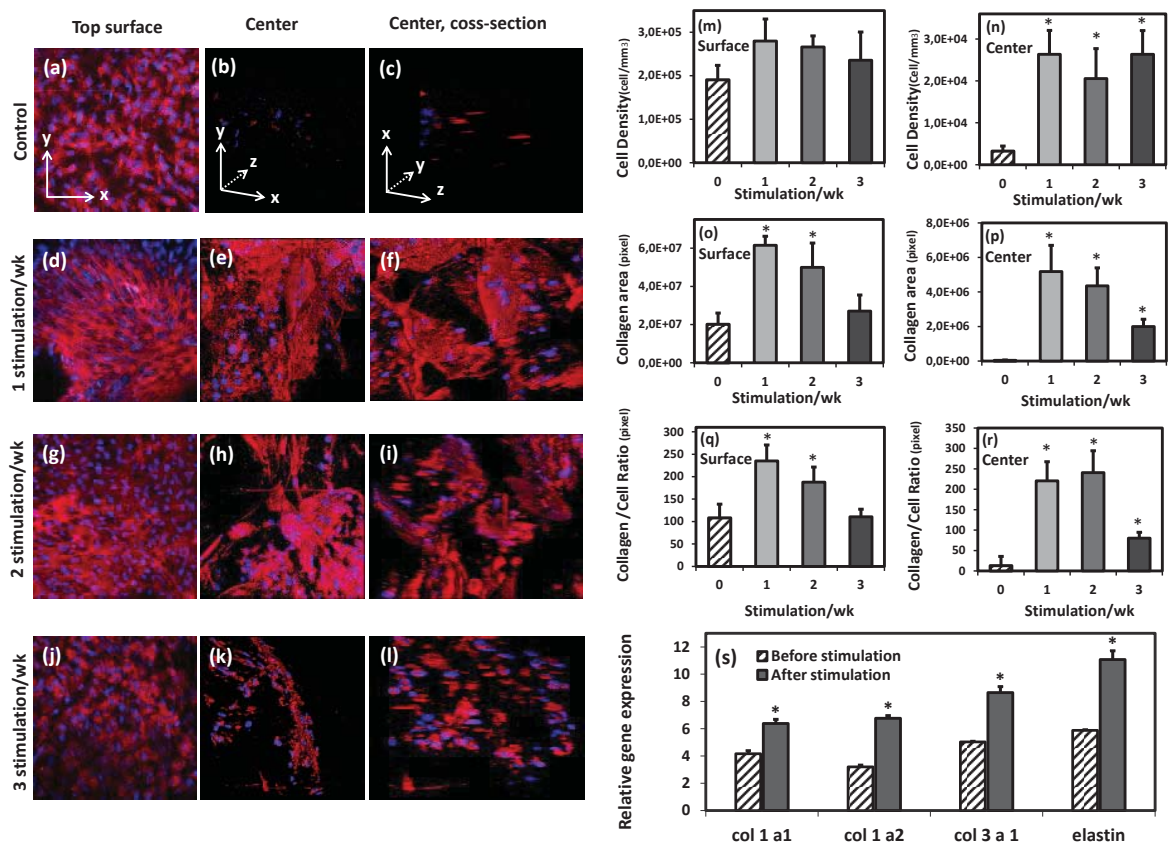

Fig. 3. two photon laser microsopy scaning images of SMCs (DAPI, blue) and Collagen organizationn (CNA-35, red) from the surface, center and center cross-section ( $z$ direction) of the non-stimulated (o stimulations/wk) (a-c) and stimulated constructs seeded with pSMCs of 1 (d-f), 2 (g-i) and 3 times stimulation (j-l) per wk, over 4 wks of culture. Total cellular density $(m, n)$, collagen area $(o, p)$ and collagen production per cell $(q, r)$ quantified at the surface and in the center cross section of hydrogels. RT-PCR analysis of gene expression of Extracellular matrix genes including Collagen 1a1, Collagen1a2, Collagen $3 a 1$ and Elastin in PAA/Fibrin hydrogel before and after the last stimulation at the 4 th wk of culture (s). Asterisks indicate $\mathrm{P}<0.05$ compared to no stimulation or before stimulation.

In accordance with previous observation in rods made from PVA/PAA hydrogels, lower bending angles were also observed with increasing gel width. [20]. However, the bending angle of hydrogels, which were exposed to one stimulation per wk stayed constant over time, although they had the same compaction ratio as samples stimulated 3 times per wk. Since collagen amount was much higher in the once per wk stimulation than 3 times per wk stimulation, the resulting mechanical strength likely resisted the bending (Correlation coefficient between bending angle and collagen production at the end of 4th wk: Pearson's $r=0.93, P<0.01)$. 
(a)

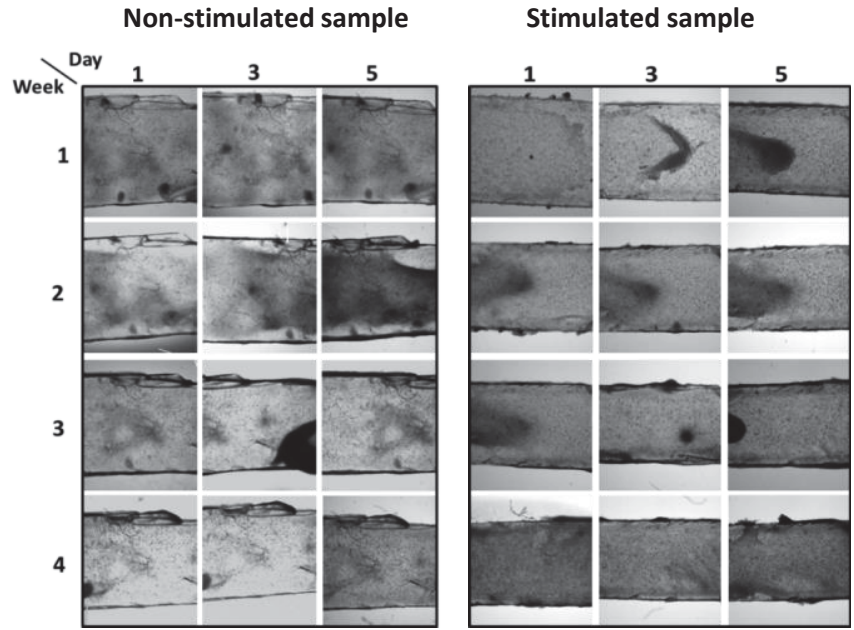

(b)

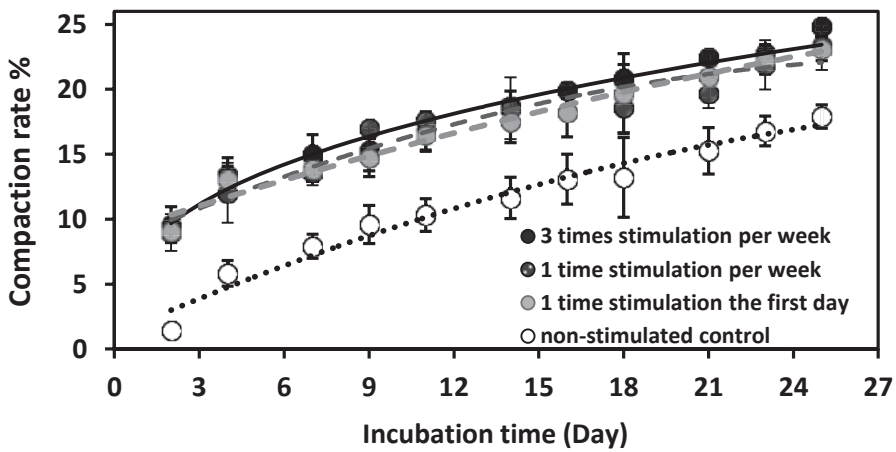

(c)

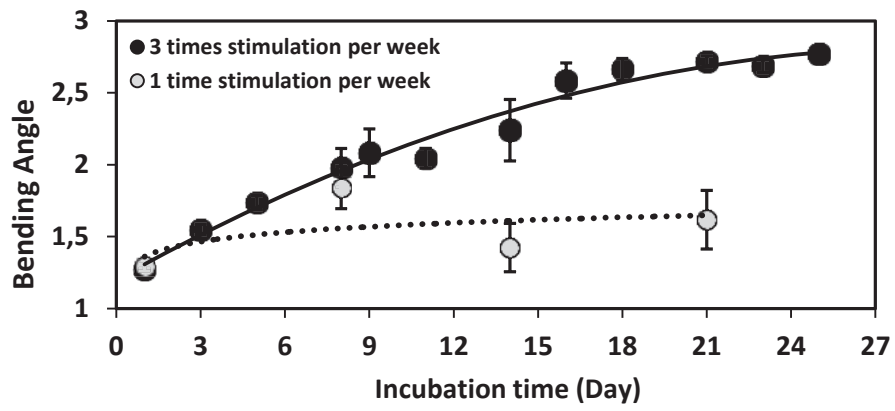

Fig. 4. Light macroscopic images of pSMCs seeded PAA/Fibrin hydrogel shows compaction of the samples in non-stimulated (control) and one stimulation per wk condition over 4 wks of culture (a), the compaction rate of non-stimulated control and stimulated samples (single stimulation on the seeding day, 1 and 3 stimulations per wk) (b), The bending angle during

stimulation time of samples stimulated 1 and 3 times per wk over 4 wks of culturing (c). 


\section{Mechanical properties of PAA/Fibrin hydrogels}

The mechanical properties of the pSMCs seeded PAA/hydrogels stimulated condition (one stimulation/wk) and non-stimulated (static) condition were characterized by performing tensile testing along the longitudinal axis of the hydrogels. In addition, non-seeded PAA/Fibrin hydrogel incubated in smooth muscle cells medium for 4 wks were tested as non-cellularized controls and were compared with seeded and stimulated samples to evaluate the effect of cell seeding and stimulation on the mechanical properties of the tissues.

The stress-strain curves of hydrogel behavior are illustrated in Fig. 5a. The maximum tensile strength (Fig. 5b) and elongation at break (Fig. 5c) were significantly increased in seeded samples, which were stimulated once per wk (26.6 kPa, 79.5\%) compared to the statically cultured seeded- (15.7 kPa, 40.8\%) and non-seeded PAA/Fibrin gels (12.5 kPa, 26.5\%). No significant difference was found for ultimate strength and elongation between the seeded and nonseeded hydrogels cultured in the static condition.

The elastic modulus was not significantly different between the stimulated, non-stimulated and non-seeded control gels (average of $45 \mathrm{kPa}$, data not shown), which likely reflects the lack of Elastin deposition in the structures. The higher collagen density in the stimulated hydrogels as a result of the even distribution of cells within the stimulated samples [8], accompanied by a great increase in collagen production per cell is likely the main reason for the increase (70\%) in maximum strength and elongation at break (94\%).
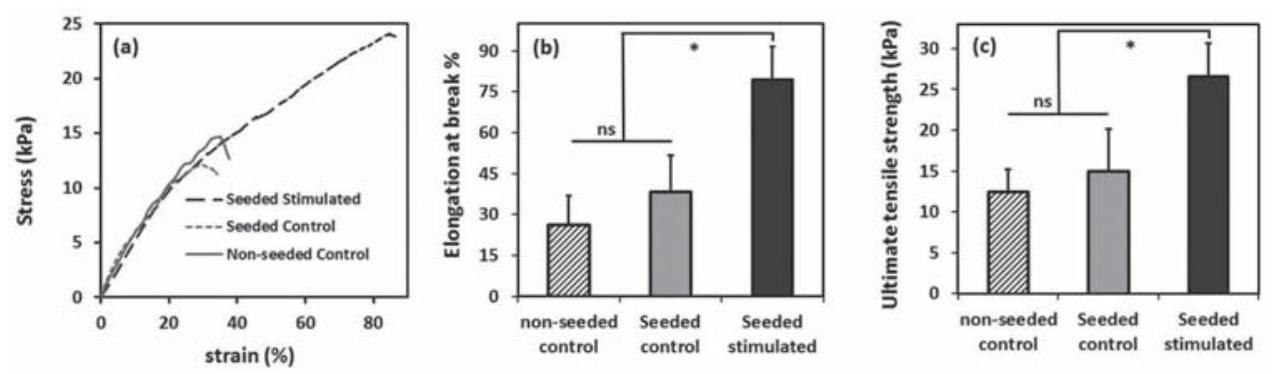

Fig. 5. Stress-stain curve (a), elongation at break (b) and ultimate tensile strength (c) of seeded and non-seeded PAA/Fibrin hydrogels cultured in stimulated and control condition under uniaxial tensile test. Asterisks indicate $\mathrm{P}<0.05$ compared to controls; ns=not significant. 


\section{MMP activity in PAA/Fibrin hydrogels during 4 wks culturing}

For the optimum stimulation protocol (1 stimulation per wk during $4 \mathrm{wks}$ ), we measured the activity of MMP-2 and MMP-9 in conditioned media of PAA/Fibrin hydrogel samples before and after every stimulation as well as in nonstimulated controls (Fig. 6). Compared to the control condition, MMP-2 activity was more than doubled after the first stimulation on the day of seeding followed by 1.6- and 1.8-fold increase after the 2nd and 3rd stimulation, respectively (Fig. 6b). The 4th stimulation no longer resulted in a significant increase in MMP-2 activity.

In the conditioned media collected from stimulated samples before stimulation and from non-stimulated controls, there was no significant difference between the levels of MMP-2. MMP-9 activity was substantially lower than MMP-2 and showed no effect of stimulation in the 1st and 2nd stimulation, while expression significantly decreased after the $3 \mathrm{rd}$ and 4th stimulation compared with before stimulation and non-stimulated control samples (Fig. 6c).

To check whether the effect of stimulation on MMP-2 activity is a result of the applied electrical field or the mechanical force, which is produced by swelling and deswelling of the PAA/Fibrin hydrogel, we studied MMP activity of pSMCs in Fibrin gel. Fibrin gel does not mechanically respond to the electrical field, therefore the effect of the electrical field on MMP-2 activity by PSMCs can be studied. The pSMCs seeded Fibrin gels were exposed to the same electrical stimulation regimes as PAA/Fibrin hydrogels (0.06 V/mm, $0.0167 \mathrm{~Hz}, 2 \mathrm{hrs}$ ) on the day of seeding and MMP-2 activity was analyzed (Fig. 6d). No stimulatory effect on MMP-2 activity was observed. In addition, to establish the relationship between electrical field induced contraction of pSMCs and MMP-2 activity, L-type voltage gated calcium channels of pSMCs were blocked by adding verapamil $(10 \mu \mathrm{M})$ just prior to stimulation. Blocking pSMCs contraction had no effect on electrical field induced MMP-2 activity in PAA/Fibrin gel. These data suggest that the applied electrical field or the resulting contraction by pSMCs are not the driving forces for the observed enhancement of MMP activity. 


\section{Discussion}

The art of tissue engineering lies in the design and complexities of the scaffold where instructive signals could be provided to the cells. In our previous work

(a)

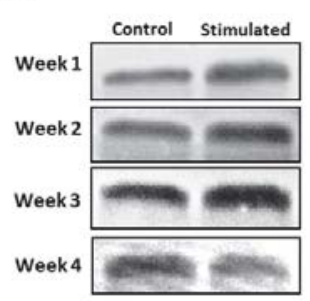

(c)
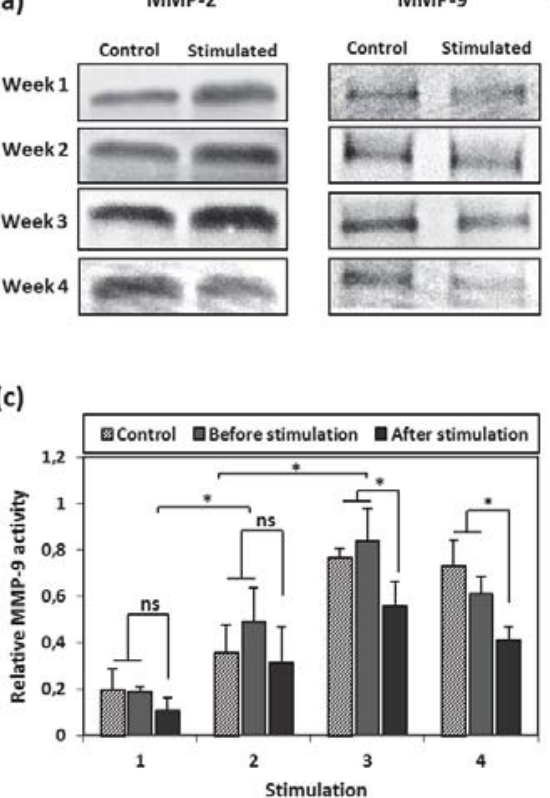

(b)

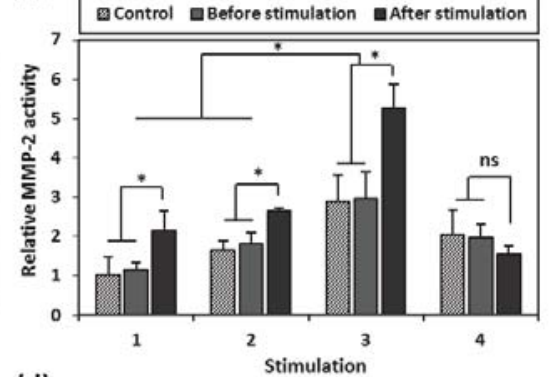

(d)

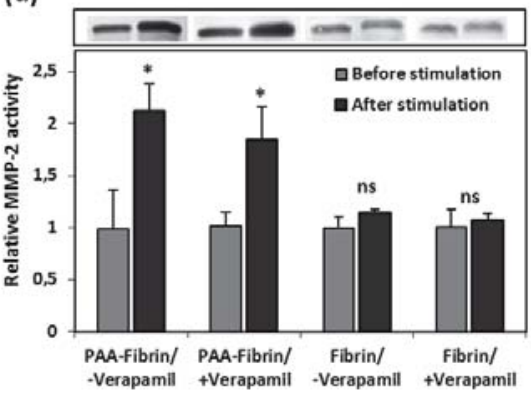

Fig. 6. Zymography gels (a) of the conditioned media represents MMP-2 (b) and MMP-9 (c) activity in the pSMCs seeded PAA/Fibrin hydrogels in static condition (control), before stimulation and after stimulation (1 stimulation per wk, 2 hrs, alternative 0.06 $\mathrm{V} / \mathrm{mm}$ ) during $4 \mathrm{wk}$ of culture. MMP-2 activity in the pSMCs seeded PAA/Fibrin hydrogels and Fibrin Gel on the first day of seeding, with or without verapamil treatment $(10 \mu \mathrm{M})$ during stimulation $(\mathrm{d})$. Asterisks indicate $\mathrm{P}<0.05$; ns= not significant.

we showed that one single electrical stimulation $(0.06 \mathrm{~V} / \mathrm{mm}, 0.0167 \mathrm{~Hz}, 2 \mathrm{hrs}$ ) applied directly after seeding the electro-sensitive hydrogels, improved the penetration of cells through the depth of the hydrogel compared to nonstimulated control samples after $1 \mathrm{wk}$ of culture [8]. Uniform cell distribution of cells within the scaffolds is the potential key to produce uniform tissue. Apart from static cell seeding techniques, various methods such as perfusion and centrifugation of cell suspension into the scaffolds have been used to 
improve the seeding efficiency [21]. In our system, due to exothermic polymerization and crosslinking reaction of acrylic acid, as well as acidic $\mathrm{pH}$ in the pre-polymerized solution, it was not possible to seed the cells during molding [22]. However the responsive swelling/deswelling of the hydrogel facilitated cell infiltration into the structure, obviating the need for cell mixing during gel molding. In the current study, we analyzed the effect of prolonged culture time up to $4 \mathrm{wks}$ for hydrogels that were stimulated $(0.06 \mathrm{~V} / \mathrm{mm}$, $0.0167 \mathrm{~Hz}, 2 \mathrm{hrs}$ ) once after cell seeding by checking for cell growth and tissue formation.

Cyclic strain plays a critical role in inducing collagen and elastin synthesis by vascular SMCs, however the responses are highly dependent on the amplitude, frequency and also the duration of the strain [4]. Other different techniques such as micropatterning, perfusion systems and ultrasound standing wave fields published recently have been shown to affect the ECM production [2325]. In our study, the mechanical forces on the cells were very briefly applied, but still resulted in increased tissue formation. Increasing the exposure time of mechanical loading to the cells ( 3 stimulations per wk) had a reduced effect on collagen production, as noted in previous studies [26].

The elevation of Transforming growth factor (TGF- $\beta$ ) in the mechanically stimulated samples tends to be the key mediator of the stretch dependent collagen production in SMCs $[27,28]$. In addition, the strain-dependent release of TGF- $\beta$ has been shown to stimulate L-proline and L-arginine transport during collagen formation and maximize the capacity of cells to synthesize collagen [29].

Enhancement of ultimate tensile strength in the SMCs seeded collagen scaffold exposed to cyclic stretching associated with higher collagen content and integrity has been reported by others $[5,26]$. We also observed higher ultimate tensile strength and elongation at break which confirm higher collagen production and tissue formation within the stimulated samples. However, tensile strengths in the kPa range are still not sufficient to meet the mechanical requirements for tissue engineered blood vessels. Either prolonged culturing or combinations with other biomaterials are options for our technology to use in vascular grafts.

Our observations on increased MMP-2 with mechanical stimulation produced within the hydrogel are in accordance with earlier studies where strain facilitated remodeling of the collagen substrate by the enhancement of MMP2 activity $[26,28,30]$. However it has been shown that the response of SMCs in regulating the pro-MMPs protein and MMP activity is dependent on the 
regimen of the applied mechanical force with increased MMP-2 activity by stationary strain and decreased MMP-2 activity by cyclic strain [31]. The low frequency of applied electrical field $(0.0167 \mathrm{~Hz})$ in our system likely produces a static strain in the hydrogel.

The balance between collagen production by cells and degradation by proteases determines collagen accumulation in tissue engineered structures [32]. Indeed, the degradation and reorganization of the collagen plays a key role in remodeling of the ECM $[33,34]$. The cellular response to mechanical forces induced in the PAA/Fibrin hydrogel has resulted in higher expression levels of MMP-2, which could lead to higher reorganization of produced collagen later during culture [35]. The improvement in strength and elongation properties of the mechanically-stimulated samples correspond well with the increasing MMP-2 activity level, which suggests that reorganization of the construct leads to an integrated collagen network [30, 36].

In addition, it has been reported that increasing MMP-2 activity is linked to SMCs migration, where MMP-2 could mediate the migration of SMCs through barriers by degrading ECM and non-matrix substrates [37]. MMP-2 and MMP-9 can also degrade Fibrin and Fibrinogen [38]. Higher penetration of cells in stimulated PAA/Fibrin hydrogel which we observed previously [8], is consistent with the higher activity of MMP-2 induced by stimulation.

The proposed system has the potential to be translated into in vivo application by mounting electrode patches on the skin and stimulating the graft in a transcutaneous way to attract cells and stimulate tissue growth. Stickler et al. investigated the effect of external signals of mechanical forces to improve the development and growth of vascular graft in Peritoneum in vivo [39]. Cells in the peritoneal cavity were attracted around a tubular scaffold by a designed cyclic stretching device connected to a pulsatile pump. Comparison between pulsed and non-pulsed scaffold revealed significantly greater collagen organization, F-actin expression and mechanical properties in pulsed scaffolds [39]. In addition, electro-responsive hydrogels have been tested as drug carriers to release drugs by swelling and deswelling under electrical stimulation [40], and therefore could be used to facilitate attracting specific cells and stimulating tissue formation by releasing chemotaxis and growth factors.

On other hand, the body immune response to the implanted graft is an important criteria related to the biocompatibility of the biomaterial. UV exposure is being used as one the classical technique to sterilize biomaterials and hydrogels before in-vivo applications [41, 42], however long UV irradiation could cause substantial changes in the polymer properties and its biological 
functionality as has been reported by Fischbach et.al [43]. Indeed, depending on the nature of the biomaterial and sterilization treatment (gamma, ethylene oxide, UV, autoclave, etc.) potential changes could be induced in molecular weight, mechanical strength and surface topography of the polymer as has been reported recently [44-46].

In addition, the biodegradability of the implanted graft should also be taken into consideration for future clinical approaches. The current hydrogel is based on covalent crosslinking of Acrylic acid monomers by $\mathrm{N}-\mathrm{N}^{\prime}$ Methylenebisacrylamide (MBAA) as the cross-linker in presence of Fibrin fibers. MBAA cross-linked acrylic acid has been reported to show low inflammatory reaction with no systemic toxicity in a rat model likely because cross-linking inhibits the solubility of the polymer and therefore reduce toxicity effects of the structure [47]. However, clinical application of these hydrogels is restricted due to their non-biodegradability. Biodegradable cross-linkers and use of natural electro-responsive biopolymers such as alginate are promising alternatives for future applications.

\section{Conclusion}

In this paper we evaluated the functionality of the electro-responsive PAA/Fibrin hydrogel to enhance tissue formation as well as to improve collagen organization in the construct. Short application of the electrical field to seeded pSMCs in PAA/Fibrin hydrogel produces a mechanical force, which efficiently induces higher collagen production, higher activity of matrix metalloprotease and higher reorganization of the collagen content in the hydrogel. The improved collagen accumulation within the structures also enhances the mechanical properties of the hydrogel significantly compared to nonstimulated control samples. With our designed hydrogel device and set-up we can facilitate and instruct 3D hydrogel tissues that could be used for tissue engineering applications, such as creating tissue engineered blood vessels in which simultaneous alignment and improved collagen production are warranted. 


\section{Acknowledgment}

This research forms part of the Project P1.01 iValve of the research program of the BioMedical Materials institute, co-funded by the Dutch Ministry of Economic Affairs. The financial contribution of the Nederlandse Hartstichting is gratefully acknowledged. This work was also supported by the Research and Expertise Center for microscopic imaging of Maastricht University, Faculty of Health, Medicine, and Life Sciences (FHML). D.G.M. is supported by the Euregio meuse-Rhine Interreg IV-A BioMIMedics project. We also thank Sheila Moli from Aachen University and Mohammad Ghomi Rostami from Sharif University of Technology for their support and assistance.

\section{Author Disclosure Statement}

No competing financial interests exist. 


\section{References:}

1. Badylak, S.F., The extracellular matrix as a scaffold for tissue reconstruction. Seminars in Cell \&amp; Developmental Biology, 2002. 13(5): p. 377-383.

2. Stock, U.A., et al., Dynamics of extracellular matrix production and turnover in tissue engineered cardiovascular structures. J Cell Biochem, 2001. 81(2): p. 220-8.

3. Chan-Park, M.B., et al., Biomimetic control of vascular smooth muscle cell morphology and phenotype for functional tissue-engineered small-diameter blood vessels. J Biomed Mater Res A, 2009. 88(4): p. 1104-21.

4. Gupta, V. and K.J. Grande-Allen, Effects of static and cyclic loading in regulating extracellular matrix synthesis by cardiovascular cells. Cardiovasc Res, 2006. 72(3): p. 375-83.

5. Kim, B.S., et al., Cyclic mechanical strain regulates the development of engineered smooth muscle tissue. Nat Biotechnol, 1999. 17(10): p. 979-83.

6. Seliktar, D., R.M. Nerem, and Z.S. Galis, Mechanical strain-stimulated remodeling of tissue-engineered blood vessel constructs. Tissue Eng, 2003. 9(4): p. 657-66.

7. Leung, D.Y., S. Glagov, and M.B. Mathews, A new in vitro system for studying cell response to mechanical stimulation. Different effects of cyclic stretching and agitation on smooth muscle cell biosynthesis. Exp Cell Res, 1977.109(2): p. 285-98.

8. Rahimi, N., et al., Electrosensitive Polyacrylic Acid/Fibrin Hydrogel Facilitates Cell Seeding and Alignment. Biomacromolecules, 2012. 13(5): p. 1448-1457.

9. Food and Drug Administration, Device Approvals and Clearances. http://www.fda.gov/MedicalDevices/ProductsandMedicalProcedures/DeviceApprov alsandClearances/PMAApprovals/ucm311892.htm, May 2012.

10. Food and drug administration, Medical devices. 21CFR888.3027, Title 21, Volume 8, Revised April 2013.

11. Khutoryanskaya, O.V., et al., Hydrogen-Bonded Complexes and Blends of Poly(acrylic acid) and Methylcellulose: Nanoparticles and Mucoadhesive Films for Ocular Delivery of Riboflavin. Macromolecular bioscience, 2013: p. n/a-n/a.

12. Plenz, G.A.M., et al., Vascular collagens: spotlight on the role of type VIII collagen in atherogenesis. Atherosclerosis, 2003. 166(1): p. 1-11.

13. Krahn, K.N., et al., Fluorescently labeled collagen binding proteins allow specific visualization of collagen in tissues and live cell culture. Analytical Biochemistry, 2006. 350(2): p. 177-185.

14. Whatling, C., W. McPheat, and E. Hurt-Camejo, Matrix Management. Arteriosclerosis, Thrombosis, and Vascular Biology, 2004. 24(1): p. 10-11.

15. Johnson, C. and Z.S. Galis, Matrix Metalloproteinase-2 and -9 Differentially Regulate Smooth Muscle Cell Migration and Cell-Mediated Collagen Organization. Arteriosclerosis, Thrombosis, and Vascular Biology, 2004. 24(1): p. 54-60.

16. Megens, R.T.A., et al., Two-Photon Microscopy of Vital Murine Elastic and Muscular Arteries. Journal of Vascular Research, 2007. 44(2): p. 87-98.

17. Boerboom, R.A., et al., High resolution imaging of collagen organisation and synthesis using a versatile collagen specific probe. Journal of Structural Biology, 2007. 159(3): p. 392-399.

18. Chiquet, M., Regulation of extracellular matrix gene expression by mechanical stress. Matrix biology : journal of the International Society for Matrix Biology, 1999. 18(5): p. 417-26. 
19. Liu, X., et al., Type III collagen is crucial for collagen I fibrillogenesis and for normal cardiovascular development. Proceedings of the National Academy of Sciences, 1997. 94(5): p. 1852-1856.

20. Shiga, T., et al., Bending of ionic polymer gel caused by swelling under sinusoidally varying electric fields. Journal of Applied Polymer Science, 1993. 47(1): p. 113-119.

21. Godbey, W.T., et al., A novel use of centrifugal force for cell seeding into porous scaffolds. Biomaterials, 2004. 25(14): p. 2799-2805.

22. Koch, S., et al., Fibrin-polylactide-based tissue-engineered vascular graft in the arterial circulation. Biomaterials, 2010. 31(17): p. 4731-9.

23. Kapoor, A., et al., Microtopographically patterned surfaces promote the alignment of tenocytes and extracellular collagen. Acta biomaterialia, 2010. 6(7): p. 2580-9.

24. Garvin, K.A., D.C. Hocking, and D. Dalecki, Controlling the Spatial Organization of Cells and Extracellular Matrix Proteins in Engineered Tissues Using Ultrasound Standing Wave Fields. Ultrasound in Medicine \& Biology, 2010. 36(11): p. 1919-1932.

25. Song, L., et al., Successful development of small diameter tissue-engineering vascular vessels by our novel integrally designed pulsatile perfusion-based bioreactor. PLoS ONE, 2012. 7(8): p. e42569.

26. Seliktar, D., R.M. Nerem, and Z.S. Galis, Mechanical strain-stimulated remodeling of tissue-engineered blood vessel constructs. Tissue engineering, 2003. 9(4): p. 657-66.

27. Li, Q., et al., Stretch-induced collagen synthesis in cultured smooth muscle cells from rabbit aortic media and a possible involvement of angiotensin II and transforming growth factor-beta. Journal of Vascular Research, 1998. 35(2): p. 93-103.

28. O'Callaghan, C.J. and B. Williams, Mechanical strain-induced extracellular matrix production by human vascular smooth muscle cells: role of TGF-beta(1). Hypertension, 2000.36(3): p. 319-24.

29. Reyna, S.V., et al., Cyclic strain stimulates I-proline transport in vascular smooth muscle cells. American Journal of Hypertension, 2004. 17(8): p. 712-717.

30. Seliktar, D., R.M. Nerem, and Z.S. Galis, The role of matrix metalloproteinase-2 in the remodeling of cell-seeded vascular constructs subjected to cyclic strain. Annals of biomedical engineering, 2001. 29(11): p. 923-34.

31. Asanuma, K., et al., Uniaxial strain upregulates matrix-degrading enzymes produced by human vascular smooth muscle cells. American journal of physiology. Heart and circulatory physiology, 2003. 284(5): p. H1778-84.

32. Solan, A., et al., Effect of pulse rate on collagen deposition in the tissue-engineered blood vessel. Tissue engineering, 2003. 9(4): p. 579-86.

33. Boccafoschi, F., et al., Biological performances of collagen-based scaffolds for vascular tissue engineering. Biomaterials, 2005. 26(35): p. 7410-7.

34. Holzapfel, G.A., Collagen in Arterial Walls: Biomechanical Aspects, in Collagen: Structure and Mechanics, P. Fratzl, Editor. 2008. p. 285-324.

35. Deryugina, E.I., et al., Remodeling of collagen matrix by human tumor cells requires activation and cell surface association of matrix metalloproteinase-2. Cancer research, 1998. 58(16): p. 3743-50.

36. Nerem, R.M. and D. Seliktar, Vascular tissue engineering. Annual review of biomedical engineering, 2001. 3: p. 225-43.

37. Newby, A.C., Matrix metalloproteinases regulate migration, proliferation, and death of vascular smooth muscle cells by degrading matrix and non-matrix substrates. Cardiovascular research, 2006.69(3): p. 614-24. 
38. Sternlicht, M.D. and Z. Werb, How matrix metalloproteinases regulate cell behavior. Annual review of cell and developmental biology, 2001. 17: p. 463-516.

39. Stickler, P., et al., Cyclically stretching developing tissue in vivo enhances mechanical strength and organization of vascular grafts. Acta biomaterialia, 2010. 6(7): p. 244856.

40. Murdan, S., Electro-responsive drug delivery from hydrogels. Journal of controlled release : official journal of the Controlled Release Society, 2003. 92(1-2): p. 1-17.

41. Mirensky, T.L., et al., Tissue-engineered vascular grafts: does cell seeding matter? Journal of pediatric surgery, 2010. 45(6): p. 1299-305.

42. Myung, D., et al., Bioactive interpenetrating polymer network hydrogels that support corneal epithelial wound healing. Journal of biomedical materials research. Part A, 2009. 90(1): p. 70-81.

43. Fischbach, C., et al., Does UV irradiation affect polymer properties relevant to tissue engineering? Surface Science, 2001. 491(3): p. 333-345.

44. Andrews, K.D., J.A. Hunt, and R.A. Black, Effects of sterilisation method on surface topography and in-vitro cell behaviour of electrostatically spun scaffolds. Biomaterials, 2007. 28(6): p. 1014-26.

45. Ahmed, M., et al., Effects of sterilization treatments on bulk and surface properties of nanocomposite biomaterials. Journal of biomedical materials research. Part B, Applied biomaterials, 2013. 101(7): p. 1182-90.

46. Hofmann, S., et al., Effect of sterilization on structural and material properties of 3-D silk fibroin scaffolds. Acta biomaterialia, 2014. 10(1): p. 308-317.

47. Burugapalli, K., V. Koul, and A.K. Dinda, Effect of composition of interpenetrating polymer network hydrogels based on poly (acrylic acid) and gelatin on tissue response: A quantitative in vivo study. Journal of Biomedical Materials Research Part A, 2004. $68 \mathrm{~A}(2):$ p. 210-218. 
Electro-responsive Hydrogel induces Collagen Production 


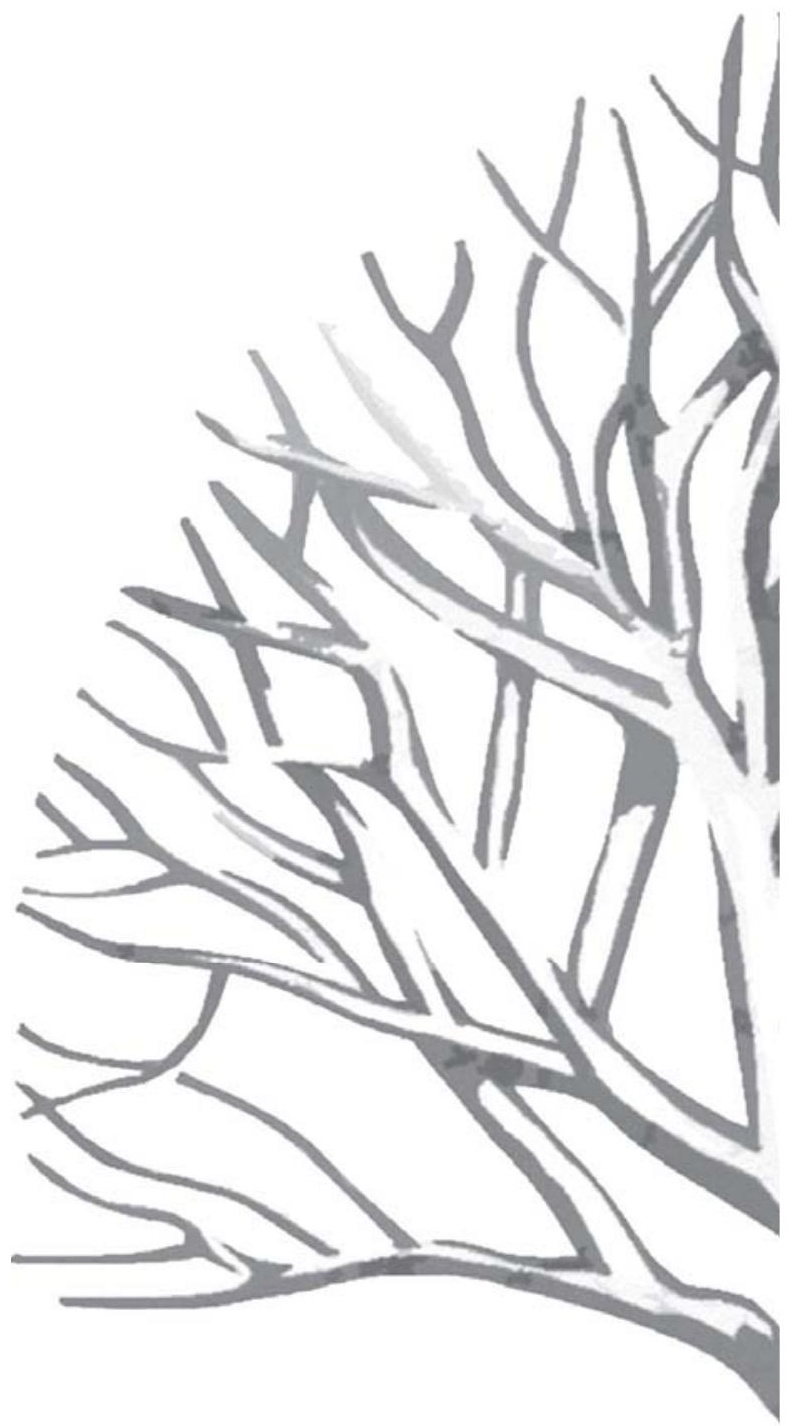




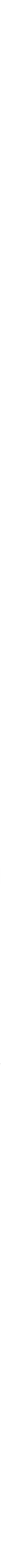

Nastaran Rahimi Geertje Swennen Martyna Scibiorek Daniel G. Molin Mark J. Post 


\section{Abstract}

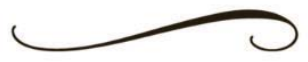

Modulation of smooth muscle cells (SMCs) morphology and phenotype are critical elements for developing functional tissue engineered vascular grafts. Previously we have reported the ability of an electro-responsive Polyacrylic acid (PAA)/Fibrin hydrogel to affect the SMCs behavior by inducing higher penetration and alignment when exposed to electrical stimulation. In the present study we assessed the regulation of SMCs morphology and phenotype when seeded on different substrates exposed to either electrical field (1 $\mathrm{mW} / \mathrm{cm} 2,0.0167 \mathrm{~Hz}, 2 \mathrm{hrs}$ ) or mechanical (5\% stretch, $0.0167 \mathrm{~Hz}, 2 \mathrm{hrs}$ ) stimulation. Fibrin fibers, SMCs nucleus and Smooth muscle actin filaments showed enhanced alignment in PAA/Fibrin hydrogels exposed to electrical and mechanical stimulation compared to non-stimulated condition. Based on our data, produced mechanical force in electrically stimulated PAA/Fibrin hydrogel is the driving force to alter topography of the hydrogel by aligning Fibrin fibers which results in alignment of cells and actin filaments. Additionally, expression of contractile proteins of SMMHC, SM- $\alpha$ actin and Calponin was significantly enhanced in SMCs seeded on PAA/Fibrin hydrogel and subsequently electrically stimulated. In contrast, electrical or mechanical stimulation of SMCs seeded in Fibrin gels did not reveal any significant difference in protein expression levels. Upregulation of SMCs contractile phenotype is likely mediated by a combination of applied electrical stimulation and produced mechanical force in PAA/Fibrin hydrogel. 


\section{Introduction}

In vivo, the phenotype of Smooth muscle cells (SMCs) is considered to match and structurally contribute to the appropriate physiological function of blood vessels. SMCs are circumferentially aligned in the medial layer of blood vessels and are continuously exposed to mechanical forces of blood pressure. These forces trigger and stabilize the contractile phenotype of SMCs in support of regulating vasomotor function during the systolic and diastolic cycle [1]. The designated contractile phenotype of SMCs phenotype is defined by the expression of several cytoskeletal contractile proteins (e.g. Smooth muscle myosin heavy chain (SMMHC), Calponin), whereas synthetic SMCs highly express extra cellular proteins (i.e. collagens) as their trade mark. The phenotype of SMC is not static and can change under altered physiological (e.g. synthetic to contractile during blood vessel development) or pathological conditions (e.g. contractile to synthetic in atherosclerosis) [2, 3].

In an ideal vascular graft, SMCs should adapt a spindle-oriented morphology with an appropriate contractile phenotype to maintain vascular tone and prevent unwanted remodeling or dysfunction of the graft (e.g. stenosis) [3, 4]. In tissue engineering, several environmental cues can be designed that induces a specific phenotype. Cell shape can be imposed through cell-substrate (i.e. ECM or graft material) interaction thus affecting the cytoskeletal microfilament forces and facilitating cell signaling and morphology [5]. Surface topography is an important substrate property to instruct cellular behavior and govern their shape $[6,7]$. This effect was first shown by Harrison in 1911, with embryonic cells following the pattern of spiders web fibers [8]. Nowadays nanotopography has been shown to induce these specific cell-substrate interactions that can alter cell adhesion, proliferation, differentiation and migration $[9,10]$. Additionally, applying external stimuli such as mechanical forces can also modulate SMCs morphology and phenotype and induce changes in ECM components and seeded substrate through biochemical responses $[11,12]$.

We previously showed a positive effect of electro-responsive Polyacrylic acid (PAA)/Fibrin hydrogel on alignment of Smooth muscle-alpha actin filaments and enhancement in production and remodeling of collagen [13, 14]. When the hydrogel is exposed to an alternating electrical field, attraction of its mobile and fixed-charged ions toward the electrodes induce structural deformation (i.e. reversible bending) in the hydrogel. In the present study, we explored the 
regulation of SMC morphology and phenotype in our developed electroresponsive hydrogel, and separated mechanical from electrical mechanisms.

\section{Materials and method}

\section{Matrix preparation}

PAA/Fibrin hydrogel and Fibrin gels were prepared as described previously [13]. Briefly, Acrylic acid (Merck, Germany) was polymerized and cross-linked in presence of Fibrin at $80{ }^{\circ} \mathrm{C}$ for $3 \mathrm{~h}$, using ammonium persulfate (APS Sigma Aldrich, The Netherlands), tetra-methylethylenediamine (TMEDA Sigma Aldrich, The Netherlands), and N, N-methylene-bisacrylamid (MBAA Sigma Aldrich, The Netherlands) as initiator, accelerator and crosslinking agent, respectively. Fibrin gel was produced by mixing Fibrinogen $(20 \mathrm{mg} / \mathrm{mL}$, Sigma Aldrich, The Netherlands) and thrombin (20 IU/mL, Sigma Aldrich, The Netherlands) solutions.

\section{Cell culturing and matrix seeding}

Primary porcine smooth muscle cells were obtained from pig aorta and cultured in fibronectin coated flask containing smooth muscle basal medium (SmBM, Lonza) including 5\% fetal bovine serum (FBS), human epidermal growth factor, human fibroblast growth factor- $\mathrm{B}$, insulin, and gentamicin. Cells were expanded until passages 8-10 under standard condition of $37{ }^{\circ} \mathrm{C}$ and $5 \%$ $\mathrm{CO}_{2}$, while changing the cell culture medium every other day.

Lyophilized, UV-sterilized PAA/Fibrin hydrogels $\left(12 \times 3 \times 1 \mathrm{~mm}^{3}\right)$ were seeded with $100 \mu \mathrm{L}$ SMCs suspension $\left(1.5 \times 10^{6}\right.$ cells/sample) dropped on the top-surface of the hydrogel. For Fibrin gels, $1.5 \times 10^{6}$ cells were first suspended in Thrombin and then mixed with Fibrinogen as described above. Fibrin solution was pipetted into the plate in a way to form a rectangular shape similar to dimensions of PAA/Fibrin hydrogels after solidification. Cell seeded substrates were incubated for 30 min at $37{ }^{\circ} \mathrm{C}$ and $5 \% \mathrm{CO}_{2}$ before adding culture SmBM medium, assuring that initial cell attachment and penetration of the hydrogel was optimal. Seeded samples were either incubated in static control or prepared for stimulation. 


\section{Electro-stimulating system}

Four-well plates equipped with carbon electrodes (Nunclon, Ion Optix, USA) were used to electrically stimulate $\mathrm{pSMC}$ seeded hydrogel matrixes as previously described [13]. Four hours after seeding, electrical field cycles of $0.06 \mathrm{v} / \mathrm{mm}$ were applied to the PAA/Fibrin hydrogels and Fibrin gels fixed at one side and solidified respectively in the middle of the well in the plate equipped with carbon electrodes (Fig. 1). The stimulation takes 2 hours and each electrical cycle consisted of $50 \mathrm{sec}$ DC current and $10 \mathrm{sec}$ off. The direction of electrical field was changed every cycle (6o sec.) by a programmable switch. Samples were then cultured under static condition $\left(37{ }^{\circ} \mathrm{C}\right.$ and $5 \% \mathrm{CO}_{2}$ ) or stimulated for another 2 hours at day 7 of culturing. Untreated non-stimulated samples were considered as controls.

\section{Strain-stimulating system}

To separate the mechanical force produced by PAA/Fibrin hydrogel in response to the electrical field, vacuum operated $\mathrm{FX}-4000^{\mathrm{TM}}$ system (Flexcell ${ }^{\circledR}$ Tension Plus System, Flexcell Corporation, McKeesport, Pennsylvania, USA) and uncoated flexible-membrane bottomed plates (Bioflex) were used. The plates were customized for straining PAA/Fibrin hydrogels and Fibrin gels by anchoring the hydrogels at both ends in a Bioflex culture plates according to description of Flexcell ${ }^{\circledR}$ Tissue Train ${ }^{\circledR}$ System for 3D culture straining. In Flexcell system, the flexible silicon membrane is stretched by a controllable vacuum system and will result in uni-axial strain of the fixed matrices. Samples were exposed to square waveform with the maximum vacuum pressure programmed and validated by the software (Flexcell International FX-4000, V4.0) to produce $5 \%$ uniaxial strain to the matrices, comparable to the calculated stretch electrical stimulation (Fig. 1a). Matrices were subjected to 2 hours of strain, four hours after seeding $\left(37^{\circ} \mathrm{C}\right.$ and $\left.5 \% \mathrm{CO}_{2}\right)$. Each strain cycle consisted of $50 \mathrm{sec}$ stretching followed by $10 \mathrm{sec}$ relaxation ( $0 \%$ strain). The strain and regime applied to the seeded constructs were determined considering the produced strain by PAA/Fibrin hydrogels when exposed to the electrical field. 

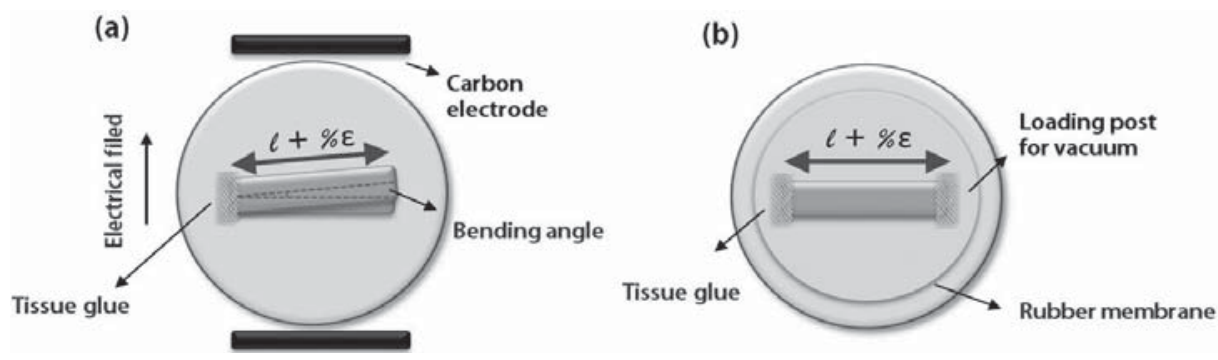

Fig. 1. (a) Schematic of electro-responsive PAA/Fibrin hydrogel bending when exposed to electrical field. (b) Strain-stimulation of PAA/Fibrin hydrogel by Flexcell system.

\section{Blocking cell contraction}

Alterations in physiological conditioning as well as applying external stimuli like mechanical and electrical forces could influence calcium influx and therefore regulate cellular cytoskeleton and contraction. L-type voltage gated calcium channels are involved in contraction of SMCs. To evaluate the direct effect of electrical field and role of calcium influx in SMCs phenotype, L-type voltage gated calcium channels as well as subsequent contraction of SMCs were blocked by adding $10 \mu \mathrm{M}$ of Verapamil (Sigma Aldrich, The Netherlands) to the culture medium of the matrices during stimulation period ( $2 \mathrm{hrs}$ ).

\section{Protein extraction and Western Blot}

SMCs seeded PAA/Fibrin hydrogel and Fibrin gels were cultured for one week either under stimulated or control condition to assess their effects on protein expression. Proteins were extracted by treating hydrogels and gels with $250 \mu \mathrm{L}$ Lysis Buffer including $10 \mu \mathrm{M}$ PBS (pH 7.2), $150 \mu \mathrm{M}$ 1\% Triton X-100 PBS, 0.1\% sodium dodecyl sulfate (SDS) and $0.5 \%$ Sodium deoxycholate [15]. To facilitate penetration of Lysis buffer into the cultured matrices, constructs were freezedried prior to protein extraction by washing with PBS followed by freezing at $20{ }^{\circ} \mathrm{C}$ overnight and freeze-drying at $4.5 \times 10^{-2}$ mbar at $-35^{\circ} \mathrm{C}$ for $48 \mathrm{~h}$. The hydrogels were then soaked in Lysis buffer and homogenized (Ultraturax) to facilitate protein release and hydrogel dissolution. Protein extracts were centrifuged and the supernatant was used to measure the concentration of protein by BCA Protein Assay Reagent (Thermo Scientific Pierce, The 
Netherlands). Protein samples were loaded on Bis-Tris Gel (4-12\% resolving gel, Criterion XT, Biorad) and transferred to PVDF membrane. To detect specific SMC protein expression, membranes were incubated with primary antibodies against Smooth muscle myosin II heavy chain (1:500,BTlinc), SM- $\alpha$ actin (1:1000, Sigma), Calponin (1:500, Sigma) and Beta-actin (1:5000, Sigma) followed by incubation with peroxidase secondary antibodies. Protein expression was detected by Pico/Femto chemiluminescence reagents (Pierce) and quantified by Quantity One ${ }^{\circledast}$ Analysis software (Bio-Rad Laboratories). For each specific protein, the expression data were normalized to beta-actin (house-keeping protein for input) and non-stimulated control samples (set at 1).

\section{Imaging}

Morphology of Fibrin(ogen) fibers in non-seeded hydrogels was visualized with FITC-labeled anti-Fibrinogen antibody (WAK Chemine, Germany, diluted 1:100 in PBS) staining. Fluorescence imaging was performed by two-photon laser scanning microscope (2PLSM, Biorad, Great Britain) coupled to a Nikon E60oFN microscope (Nikon, Tokyo, Japan). The excitation source of the microscope was a 140-fs-pulsed Ti:sapphire laser (Spectra Physics Tsunami, Mountain View, CA) tuned and mode-locked at $800 \mathrm{~nm}$ (for visualization of fluorescent probes)[16].

To evaluate the seeded SMCs morphology and characteristics, samples were cultured for $1 \mathrm{wk}$, washed with PBS, fixed in Paraformaldehyde (PFA 2\%) for 20 min at $4{ }^{\circ} \mathrm{C}$ and stained with smooth muscle (SM)-a-actin-FITC antibody (Sigma, diluted 1:250 in PBS) at $4{ }^{\circ} \mathrm{C}$ overnight and subsequently, DAPI (Sigma, diluted 1:1000 in PBS) for $15 \mathrm{~min}$ at room temperature. Hydrogels were then fixed in $2 \%$ Agarose gel for 2 PLSM scanning. Images were recorded with $40 \mathrm{x}$ objective with $2.0 \mathrm{~mm}$ working distance (numerical aperture (NA) 0.8, Nikon) in $\mathrm{x}-\mathrm{y}$ plane. ImageJ software (NIH, Bethesda, MD) was used to access collected images. Orientation of cell nuclei was quantified by the Directionality plug in (author: Jean-Yves Tinevez, http://pacific.mpicbg.de/wiki/index.php/Directionality) using Fiji software. Data were presented as Directionality histogram. The histogram indicates the ratio of the structure which is aligned in the given direction, normalized to the total area of the structure (from $-90^{\circ}$ to $+90^{\circ}$ ). The area below the histogram is considered 1 for all the samples. The preferred orientation degree in each condition ( $>9$ images per condition), is indicated by the highest peak in the Gaussian curve fitted to the histogram. The relative oriented cell nucleus were calculated by measuring 
the percentage of area from peak value-SD to peak value+SD divided to total value of the histogram indicating zero (o) for completely isotropic sample. The alignment of SM-a-actin filaments in the samples was quantified by the Orientation Image-J plugin (Daniel Sage, EPFL, Switzerland, http://bigwww.epfl.ch/demo/orientation). Data were presented as polar graph of orientation distribution frequency for each condition $\left(0^{\circ}\right.$ to $\left.360^{\circ}\right)$. The fiber orientations were quantified for each condition considering the induced stress direction in the hydrogel as the reference line.

\section{Statistics}

Data were expressed as means with standard deviation for each condition. To compare groups' means statistical analysis was performed by t-test (Graphpad prism 4, Graphpad Prism Software Inc., San Diego, USA). P-values <0.05 were considered to be significant.

\section{Results}

\section{Fibrin fiber orientation}

To determine changes in morphology of the PAA/Fibrin hydrogel structure, orientation of Fibrin fibers were evaluated by staining Fibrin with AntiFibrin(ogen) fluorescent antibody (Fig. 2). Hydrogels exposed to electrical or mechanical stimulation ( 2 hrs) showed profound alignment of Fibrin fibers along the stress direction (Fig. 2 a,b). Fibrin fibers in samples treated under control condition with no stimulation revealed random orientation with no preferred direction (Fig. 2c). Induced alignment of Fibrin fibers in PAA/Fibrin hydrogels after exposure to stimulation could provide a patterned substrate, which causes cells to elongate accordingly.

\section{Alignment of cells and Alpha-actin filaments}

Smooth muscle cells alignment is an essential key for proper functionality of blood vessel by providing vasodilation of the vascular wall [17]. Cell nucleus and SM-alpha actin filaments orientation were analyzed in SMCS PAA/Fibrin seeded hydrogels that were either electrically $(1 \mathrm{~mW} / \mathrm{cm} 2,0.0167 \mathrm{~Hz}, 2 \mathrm{hrs}$ ) or mechanically ( $5 \%$ stretch, $0.0167 \mathrm{~Hz}, 2$ hrs) stimulated in the first day of seeding. 


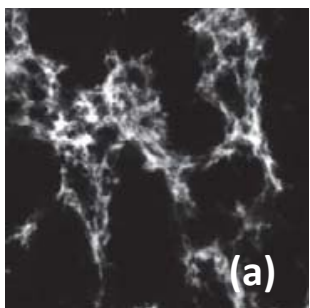

S/EC- PAA/Fibrin

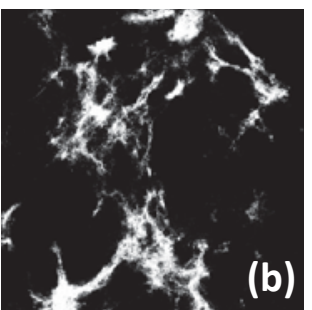

S/ME- PAA/Fibrin

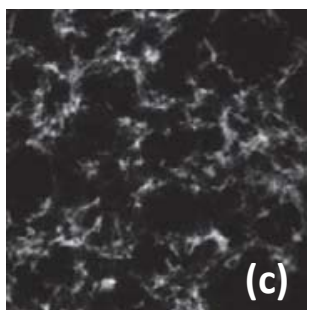

Con- PAA/Fibrin

Fig. 2. Two-photon microscopy images of Fibrin fibers orientation in PAA/Fibrin hydrogels showed alignment of Fibrin fibers when exposed to electrical field (EC) (a) or mechanical stretch (ME) (b) stimulation and non-stimulated control condition (con) (c).

Additionally, PAA only hydrogels were also electrically stimulated to study the role of Fibrin structure on cell alignment. PAA/Fibrin hydrogels cultured in static condition (no stimulation) were considered as controls. Samples were cultured for 1 week before being visualized by two photon laser scanning microscopy (2PLSM) to determine SMCs nucleus.

Electrically and mechanically simulated PAA/Fibrin hydrogels showed aligned nucleus in parallel direction of aligned Fibrin fibers and induced stress. While PAA only hydrogels exposed to electrical field and non-stimulated PAA/Fibrin hydrogels showed no significant alignment of the nucleus orientation (Fig. 3 ad). Distribution of cell nucleus orientation in the representative 2PLSM images of each condition is illustrated by directionality histograms ranging from $-90^{\circ}$ to $90^{\circ}$ (Fig. 3 e-h). The spread in orientation angle was much higher in the absence of Fibrin and in the non-stimulated controls than in the mechanically or electrically stimulated PAA/Fibrin hydrogels (Fig. 3i). PAA/Fibrin hydrogels exposed to electrical and mechanical stimulation showed approximately $60 \%$ of nuclei aligned in all the samples (Fig. 3j).

The dispersion of oriented area in stimulated PAA and non-stimulated PAA/Fibrin were at least three times higher than stimulated PAA/Fibrin hydrogels, indicating heterogeneous cell orientation in these samples.

Alignment of SM- $\alpha$ actin filaments in SMCs seeded hydrogels were also evaluated based on 2 PLSM images of the samples after 1 week of culture (Fig. 4 a-d).

Color coded maps illustrate the overall distribution of fiber direction by attributing a color to each alignment angle (Fig. 4 e-h). 

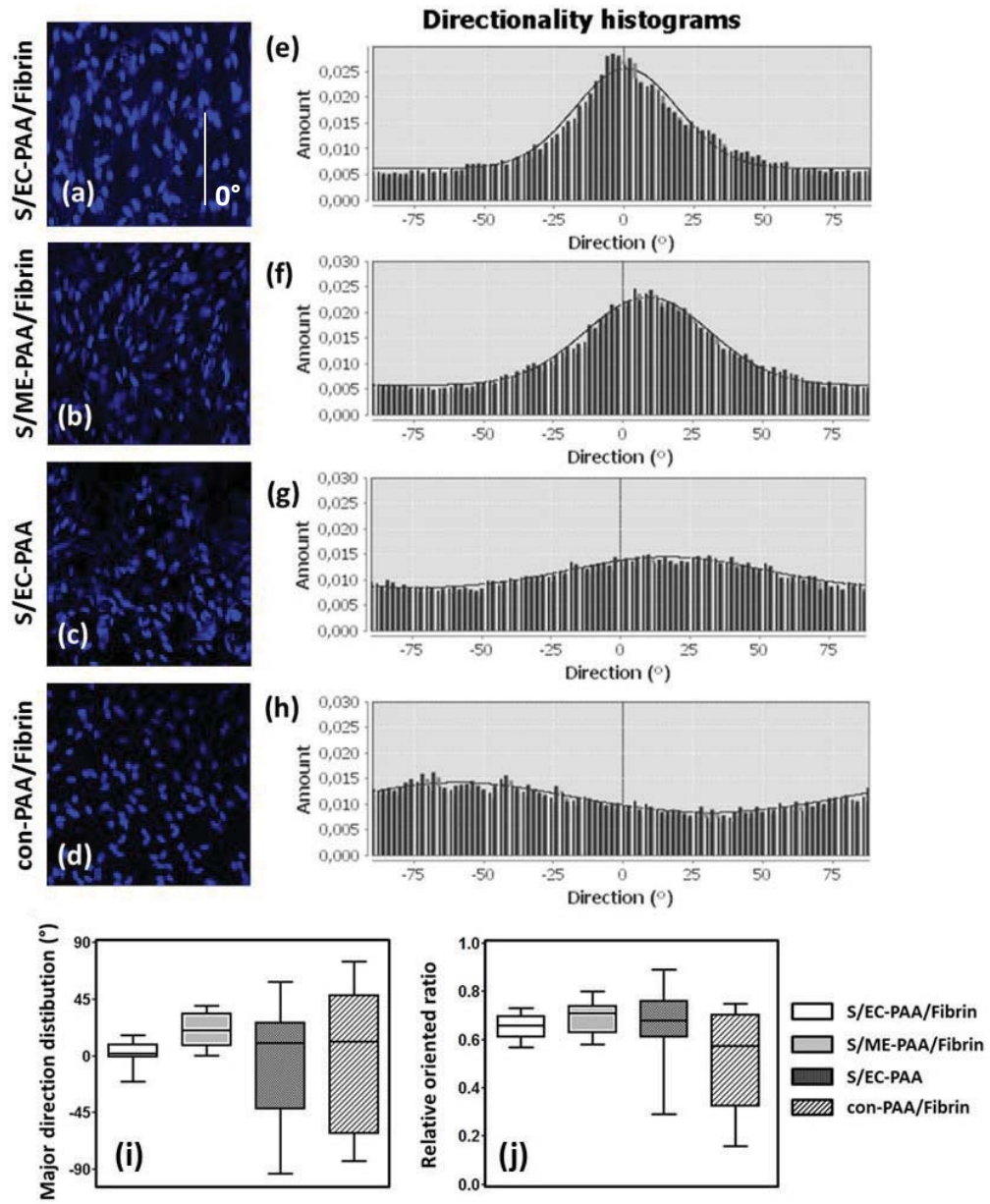

Fig. 3. Two-photon laser scanning microscopy images of smooth muscle cell nucleus (DAPI, Blue) and directionally histogram of PAA/Fibrin electrically stimulated (S/EC)(a,e), PAA/Fibrin mechanically stretched $(S / M E)(b, f), P A A$ electrically stimulated $(c, g)$ and nonstimulated control (con)(d,h) PAA/Fibrin hydrogels after 1 week culturing. Graph (i) shows the distribution of preferred alignment angle of the aforementioned samples and graph (j) represents the ratio aligned nuclei to total number of nuclei.

PAA/Fibrin hydrogels exposed to electrical stimulation $(1 \mathrm{~mW} / \mathrm{cm} 2,0.0167 \mathrm{~Hz}, 2$ hrs) after seeding showed elevated alignment of SM-alpha-actin filaments along the width of the hydrogel parallel to induced stress direction after 1 week of culture (Fig. 4 a,e,i). 

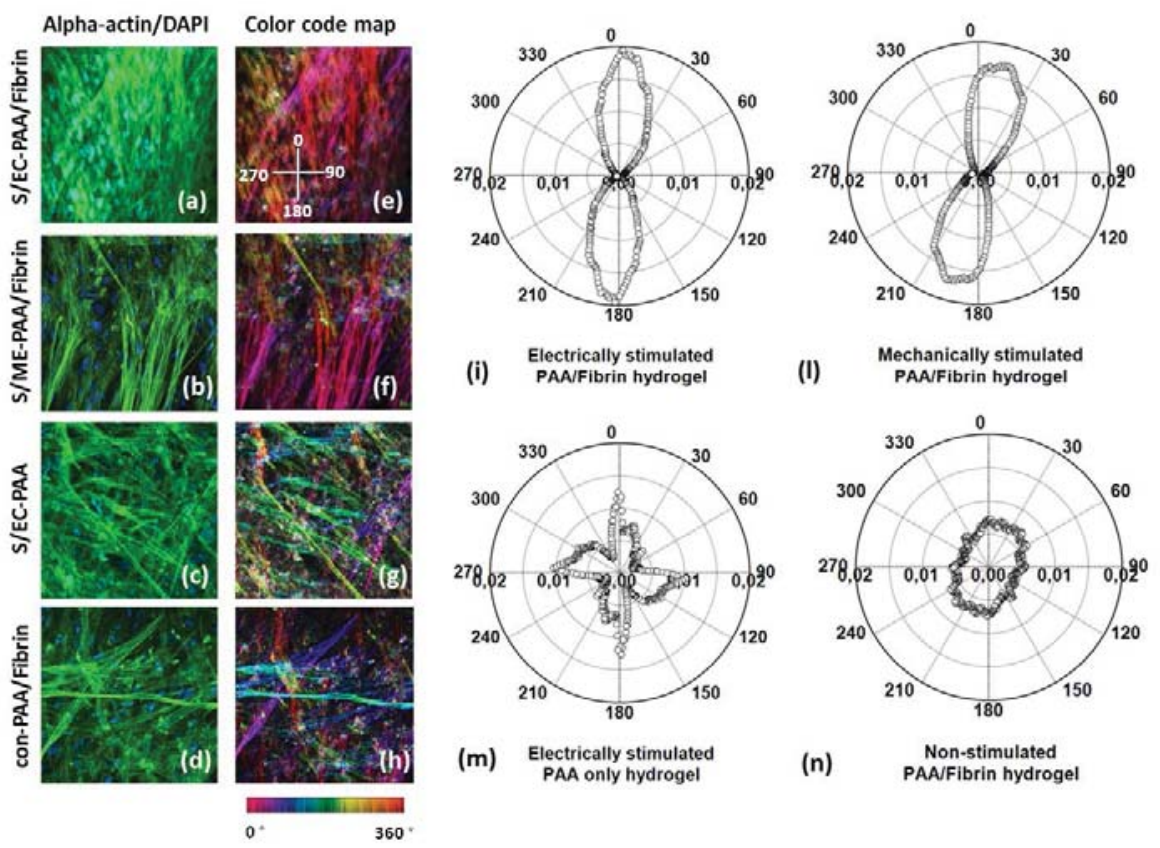

(m) Electrically stimulated

Non-stimulated
PAA/Fibrin hydrogel

Fig. 4. Two-photon laser scanning microscopy of smooth muscle cell nucleus (DAPI, Blue) and alpha-actin filaments (green), color-coded map of actin filaments and polar graphs of overall orientation distribution of actin filament in PAA/Fibrin electrically stimulated $(\mathrm{S} / \mathrm{EC})(\mathrm{a}, \mathrm{e}, \mathrm{i}), \mathrm{PAA} /$ Fibrin mechanically stretched $(\mathrm{S} / \mathrm{ME})(\mathrm{b}, \mathrm{f}, \mathrm{I}), \mathrm{PAA}$ electrically stimulated $(c, g, m)$ and non-stimulated control $(d, h, n)$ PAA/Fibrin hydrogels after 1 week culturing.

In mechanically stimulation, where hydrogels were exposed to 5\% stretch after seeding $(0.0167 \mathrm{~Hz}, 2 \mathrm{hrs})$, fibers also aligned across the hydrogel and parallel to the direction of applied stress (Fig. 4 b,f,I), whereas PAA hydrogels exposed to electrical stimulation had 2 major orientation directions parallel and perpendicular to the stress direction (Fig. 4 c,g,m). In non-stimulated controls, fibers had a random orientation (Fig. 4 d,h,n).

The alignment direction of SM-alpha-actin filaments in both electrically and mechanically stimulated samples revealed high preference of actin filaments alignment along the Fibrin fibers direction. In contrast, in the electrically stimulated PAA hydrogel with no fibrin component and in non-stimulated PAA/Fibrin hydrogels with randomly dispersed Fibrin, actin filaments had no preferential orientation (Fig. $4 \mathrm{~m}, \mathrm{n}$ ). 


\section{Expression of smooth muscle contractile proteins}

To ascertain the effect on SMC phenotype, hydrogel samples were exposed to 2 hrs mechanical or electrical stimulation at day 1 , four hours after seeding and day 7 of culture, followed by Western Blot analysis of SMMHC, SM-alpha actin and Calponin proteins. Smooth muscle myosin heavy chain is a smooth muscle cell specific marker indicative of a contractile phenotype [18]. Calponin and SMa actin have been shown to be present in both contractile and synthetic phenotype, however higher expressions of these markers suggest a contractile phenotype [18].

Contractile proteins of SM- $\alpha$ actin, Calponin and SMMHC were detected at 42 $\mathrm{kDa}, 36 \mathrm{kDa}$ and $220 \mathrm{kDa}$, respectively (Fig. 5). SMCs in electrically stimulated PAA/Fibrin hydrogels expressed 10-fold higher levels of SM- $a$-actin, 8-fold higher Calponin and 7-fold higher SMMHC than non-stimulated PAA/Fibrin hydrogels (Fig. 5a). Blocking voltage gated Calcium channels by adding Verapamil inhibited the electrical stimulation-induced expression of SMMHC in PAA/Fibrin hydrogels, but not the other contractile markers (Fig. 5c). Fibrin samples exposed to electrical stimulation and Verapamil treatment did not show any significant differences in expression compared with nonstimulated ones (Fig. 5 b, d).

To further investigate the role of mechanical stress on protein expression, SMCs seeded PAA/Fibrin hydrogels and Fibrin gels were exposed to mechanical stretch stimulation $(5 \%, 0.067 \mathrm{~Hz}, 2 \mathrm{hrs})$ without and with Verapamil treatment. The extent of mechanical stress was the same as the stretch induced by electrical stimulation (Fig. 1a). Protein expressions in PAA/Fibrin hydrogels were undetectable by Western Blot after 1 week of culture, probably due to a substantially lower amount of cells and subsequently low protein in the bulk of the hydrogel (data not shown), illustrating the lack of cell penetration in the absence of electrical stimulation. Stretching of SMCs in Fibrin gels however did not affect $S M M H C$ and $S M$-a-actin protein expression irrespective of the presence of Verapamil (Fig. 6). However, Verapamil treatment significantly decreased the already very low Calponin expression in mechanically stretched Fibrin gels compared with non-stimulated samples (Fig. 6b). 
(a)

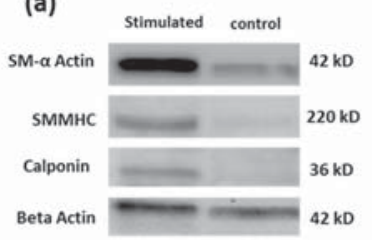

(b)

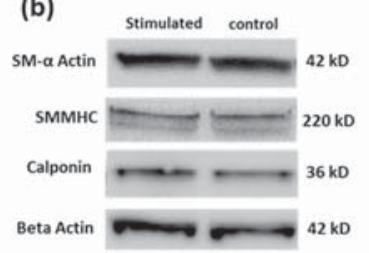

(c)

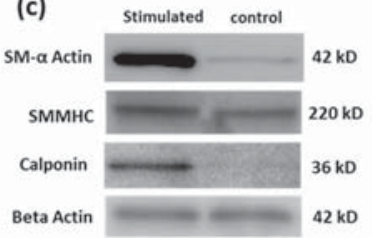

(d)

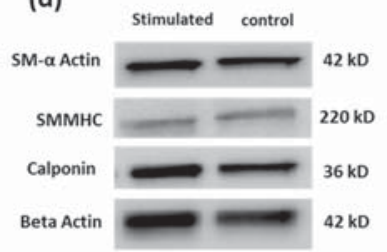

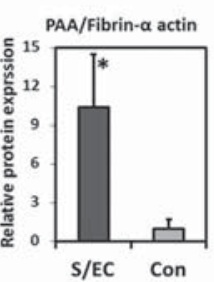
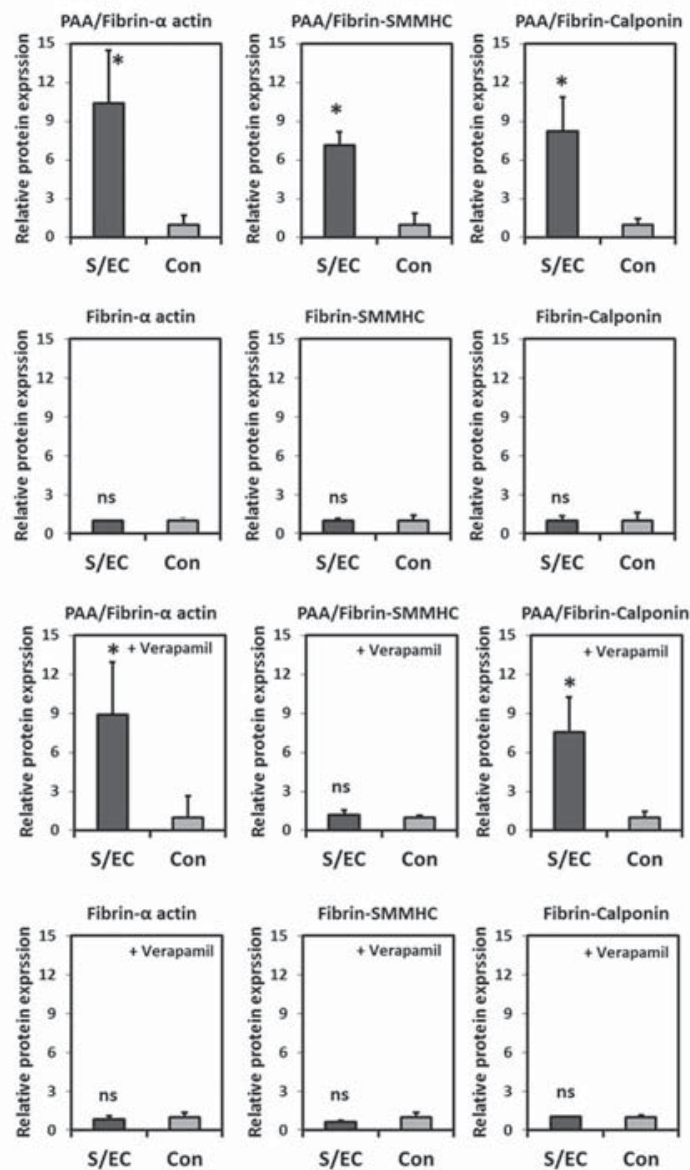

Fig. 5. Western Blot analysis of smooth muscle cell contractile protein expression for electrically stimulated (S/EC) and non-stimulated (con) PAA/Fibrin hydrogels (a,b) and Fibrin gels (c,d) without and with Verapamil (L-type calcium channels blocker, 10mM, added during stimulation time). Samples were cultured for 1 week. Asterisk indicates $P$ value $<0.05$ and $n s$ P value $>0.05$.

\section{Discussion}

When a PAA/Fibrin hydrogel seeded with SMCs is exposed to electrical stimulation a series of events occur in the hydrogel structure, which affects cell behavior. Mechanical stretching of the hydrogel structure (bending) to the negative electrode during each electrical cycle is directly related to the 
electrical responsive acrylic acid part of the hydrogel [19]. This macroscopic deformation is caused at the molecular level of the poly acrylic acid network with each carboxylic group repelling the negatively charged neighbor groups [20]. In this study we investigated the role of the two main forces of the applied electrical field and induced mechanical forces in the hydrogel on SMCs alignment and phenotype.
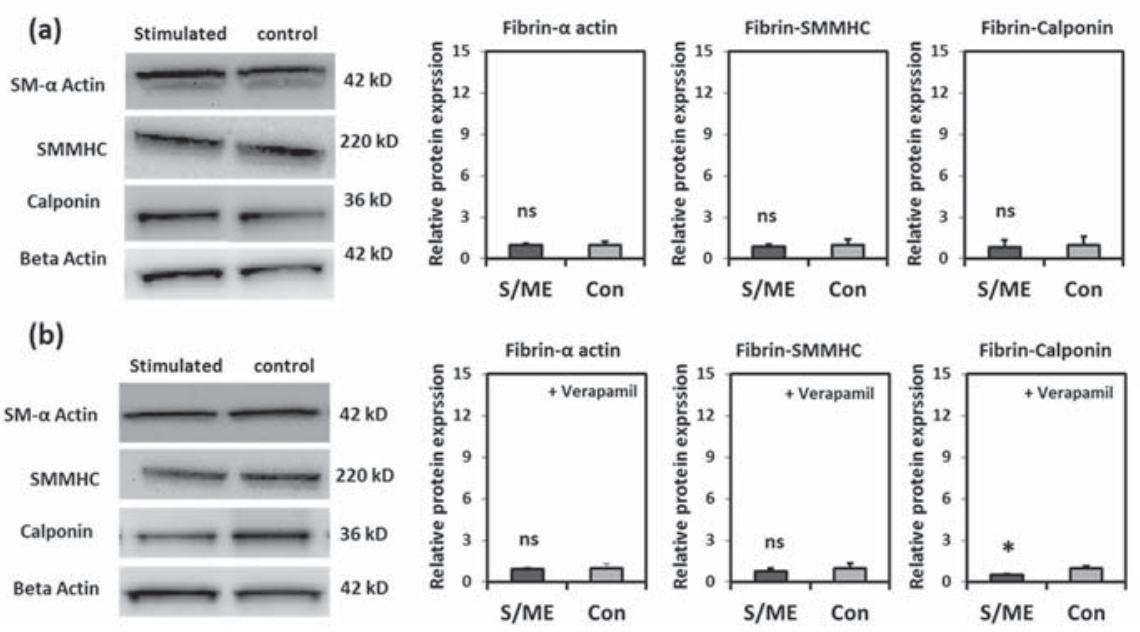

Fig. 6. Western Blot analysis of smooth muscle cell contractile protein expression of mechanically stimulated (S/ME) and non-stimulated (Con) Fibrin gels treated without (a) and with L-type calcium channels blocker (10mM Verapamil adding during stimulation time) (b). Samples were cultured for 1 week. Asterisk indicates $P$ value $<0.05$ and ns $P$ value $>0.05$.

For the transduction of mechanical force on SMCs, we consider two plausible mechanisms. The first involves direct effect of mechanical stretching on SMCs and the second is related to internal changes of hydrogel topography and especially Fibrin fiber orientation. The latter is of great importance as topographical features of biomaterials can markedly affect cell geometry and morphology [7, 21, 22].

Electrical stimulation provided a patterned substrate with high alignment of Fibrin fibers in PAA/Fibrin hydrogels. Fibrin fibers also presented high alignment parallel to stress direction when PAA/Fibrin hydrogels were exposed to mechanical stimulation. Therefore, we conclude that either induced stress by electrical stimulation (bending) or mechanical stretch of PAA/Fibrin hydrogels is the driving force for rearranging of Fibrin fibers in the structure. 
The created Fibrin micropatterning of the biomaterials allows the cells to adhere and subsequently spread along its structure and topography [23]. Random orientation of cells and actin filaments in stimulated PAA hydrogels without Fibrin also confirmed the dominant instructive role of Fibrin fiber patterning in dictating cell geometry.

Patterned fiber reinforced scaffolds have been shown to induce directionally dependent cell behavior in tissue engineered structures [24, 25]. As an example, cell shape and elongation are determining elements in regulating SMCs phenotype and functionality [26], likely mediated by focal adhesion sites with integrins and proteoglycans [27]. Chang et al. reported recently phenotype regulation of vascular SMCs induced by elongation after short culture on micropatterned substrate [28]. We also found increased SMMHC, Calponin and SM- $\alpha$ actin proteins expression in electrically stimulated PAA/Fibrin hydrogels compared with controls, indicating a phenotype transition into a more contractile state. Indeed, induced alignment of cells by contact guidance between cells and substrate could alter cytoskeletal proteins through rearrangement of focal adhesion sites [28, 29]. In addition, the tension generated by cells in response to topological cues of the matrix could modulate their phenotype and contractility [30]. However, in a Fibrin gel presumably due to highly entangled fibers no alignment could be forced by mechanical stimulation as occurred in PAA/Fibrin hydrogel. Accordingly, in Fibrin gels, no effect of either stimulation was observed, suggesting that electrical and mechanical stretch stimulation were not sufficient enough to independently modulate the phenotype in SMCs. Our results therefore suggest that spatial cues determined through Fibrin fibers as a result of conformational changes of PAA/Fibrin hydrogel under electrical stimulation could be the key mediator of phenotypic modulation of SMCs.

To examine the effect of Calcium influx in SMCS [31] and related signaling on SMCs differentiation [32] we blocked the activity of L-type calcium channels by adding Verapamil during stimulation. In PAA/Fibrin hydrogels, Verapamil only affected the SMMHC expression and did not alter SM- $\alpha$-actin and Calponin expression. This finding suggests that upregulation of SM- $\alpha$ actin and Calponin are mediated through a calcium-independent pathway whereas SMMHC expression is calcium dependent when PAA/Fibrin hydrogels are electrically stimulated. This is supported by several studies. Rowlands et al showed greater influence of blocking L-type voltage gated channels on suppressing SMMHC expression compared to $\alpha$-actin of electrically stimulated SMCs seeded on a conductive polypyrrole substrate [33]. A study by Heidkamp et al. on the 
role of Calcium transients and mechanical strain on cellular shape and localization of Myosin heavy chain (MHC) in cardiomyocytes showed calcium and not the mechanical force controls MHC mRNA localization, whereas cellular shape is regulated by structure alignment and applied stretch [34]. Wamhoff et al. provided evidence on the role of voltage gated calcium channel activation on expression of contractile markers of SMMHC and SM- $\alpha$ actin through mechanisms mediated by RhoA/Rho kinase (ROK) and myocardin [35]. Together, these findings suggest a greater influence of intracellular Ca fluxes on MHC than the other contractile proteins and that this effect may be dependent to stimulation conditions. However, in Fibrin gels stimulated mechanically in presence of Verapamil, Calponin showed significant decreased while SMMHC and SM- $\alpha$ actin proteins stayed unchanged. Calponin is located on actin bundles and partly co-localize with myosin [36]. Similar to our findings, Ren et al. also showed down regulation of Calponin when vascular SMCs were mechanically stretched in presence of Verapamil [37].

Recently, we reported a long term effect of electrical stimulation on collagen production in SMCS seeded PAA/Fibrin hydrogels [14], with 1 stimulation per week giving profound collagen production, collagen gene expression and remodeling after 4 weeks, which mostly present a synthetic phenotype of SMCs. In the presented study focusing on markers of SMC phenotype, we observed an elevated contractile phenotype of SMCs in stimulated samples. Although the behavior of SMCs in long term culturing seems to contradict with traditional phenotype classification, this apparently inconsistence response of SMCs has also been described by others $[33,38,39]$. The idea that smooth muscle cells can present spectrum of synthetic and contractile phenotype in response to functional demands has been initiated for more than 5 decades [40]. Smooth muscle cells exposed to cyclic stretch by the Flexcell system presented a dual effect of mechanical stimulation causing a pronounced increase in proliferation and Caldesmon expression, which are attributed to synthetic and contractile phenotype respectively [41]. Similarly, a recent study by Chang et al. showed an intermediate phenotype of SMCs when cultured on patterned structures [28]. In addition, changes in substrate composition and produced ECM components by cell themselves over time could also induce contractile and synthetic characteristics resulting in a mixed population of cells in the structure [21]. Also doubling times could influence reversibility of SMCS phenotype [42]. The intrinsic capacity of SMCs to phenotypically shift can be beneficial for vascular tissue engineering and provides a strong tool to control the functionality of the graft structure. Where increase in SMC migration and 
ECM (i.e. collagen) deposition -defined as synthetic SMC phenotype- support the development of a homogenous tissue with wall strength and appropriate mechanical properties, a contractile phenotype provides options for vasomotor function of the vascular grafts [43].

\section{Conclusion}

In the presented study we showed the ability of electro-responsive PAA/Fibrin hydrogel to upregulate the contractile phenotype of seeded smooth muscle cells when shortly exposed to electrical stimulation. The absence of an effect on SMC phenotype in mechanically and electrically stimulated Fibrin gels suggests that the induced deformation of Poly acrylic acid part of the hydrogel by electrical field is likely the instructive key to modulate cells phenotype. Mechanical force induced within the hydrogel affects the Fibrin fibers pattern and subsequently regulates cell arrangement, actin cytoskeleton and fate of the cells. Our developed system could be used as a strong tool to navigate phenotype plasticity of smooth muscle cell for tissue engineering application as both synthetic and contractile phenotypes are required to develop a functional vascular graft.

\section{Acknowledgment}

This research forms part of the Project WP2 iValve of the research program of the BioMedical Materials institute, co-funded by the Dutch Ministry of Economic Affairs. The financial contribution of the Nederlandse Hartstichting is gratefully acknowledged. This work was also supported by the Research and Expertise Center for microscopic imaging of Maastricht University, Faculty of Health, Medicine, and Life Sciences (FHML). D.G.M. is supported by the Euregio meuse-Rhine Interreg IV-A BioMIMedics project. We also thank Frans van Nieuwenhoven from Physiology Department at Maastricht University and Mohammad Ghomi Rostami from Sharif University of Technology for their support and assistance.

\section{Author Disclosure Statement}

No competing financial interests exist. 


\section{References:}

1. Thakar, R.G., et al., Regulation of vascular smooth muscle cells by micropatterning. Biochemical and biophysical research communications, 2003. 307(4): p. 883-90.

2. Yamin, R. and K.G. Morgan, Deciphering actin cytoskeletal function in the contractile vascular smooth muscle cell. The Journal of physiology, 2012. 590(Pt 17): p. 4145-54.

3. Stegemann, J.P., H. Hong, and R.M. Nerem, Mechanical, biochemical, and extracellular matrix effects on vascular smooth muscle cell phenotype. Journal of applied physiology, 2005. 98(6): p. 2321-7.

4. Isenberg, B.C., C. Williams, and R.T. Tranquillo, Small-diameter artificial arteries engineered in vitro. Circulation research, 2006. 98(1): p. 25-35.

5. Opal, M., Substratum mechanics and cell differentiation, in Mechanical Engineering of the Cytoskeleton in Developmental Biology, R. Gordon, Editor. 1994. p. 119-137.

6. Kim, B.S., et al., Cyclic mechanical strain regulates the development of engineered smooth muscle tissue. Nat Biotechnol, 1999. 17(10): p. 979-83.

7. Jiang, Y., S. Lu, and Y. Zeng, Dermal fibroblast behaviour on micropatterned substrates with different pattern geometries. Journal of tissue engineering and regenerative medicine, 2011. 5(5): p. 402-9.

8. Harrison, R.G., On the Stereotropism of Embryonic Cells. Science, 1911. 34(870): p. 27981.

9. Ravichandran, R., et al., Effects of nanotopography on stem cell phenotypes. World journal of stem cells, 2009. 1(1): p. 55-66.

10. Zorlutuna, P., P. Vadgama, and V. Hasirci, Both sides nanopatterned tubular collagen scaffolds as tissue-engineered vascular grafts. Journal of tissue engineering and regenerative medicine, 2010. 4(8): p. 628-37.

11. Williams, B., Mechanical influences on vascular smooth muscle cell function. Journal of hypertension, 1998. 16(12 Pt 2): p. 1921-9.

12. Bishop, J.E. and G. Lindahl, Regulation of cardiovascular collagen synthesis by mechanical load. Cardiovascular research, 1999. 42(1): p. 27-44.

13. Rahimi, N., et al., Electrosensitive polyacrylic acid/fibrin hydrogel facilitates cell seeding and alignment. Biomacromolecules, 2012. 13(5): p. 1448-57.

14. Rahimi, N., et al., Short stimulation of electro-responsive PAA/Fibrin hydrogel induces collagen production. Tissue Eng Part C Methods, 2013.

15. Seliktar, D., R.M. Nerem, and Z.S. Galis, Mechanical strain-stimulated remodeling of tissue-engineered blood vessel constructs. Tissue engineering, 2003. 9(4): p. 657-66.

16. Megens, R.T.A., et al., Two-Photon Microscopy of Vital Murine Elastic and Muscular Arteries. Journal of Vascular Research, 2007. 44(2): p. 87-98.

17. Li, Y., et al., Engineering cell alignment in vitro. Biotechnology Advances, 2014. 32(2): p. 347-365.

18. Vorp, D.A., T. Maul, and A. Nieponice, Molecular aspects of vascular tissue engineering. Front Biosci, 2005. 10: p. 768-89.

19. Kim, B.-S., et al., Engineered Smooth Muscle Tissues: Regulating Cell Phenotype with the Scaffold. Experimental Cell Research, 1999. 251(2): p. 318-328.

20. Yang, S., et al., Electroresponsive Behavior of Sodium Alginate-g-Poly (acrylic acid) Hydrogel Under DC Electric Field. Journal of Macromolecular Science, Part A, 2009. 46(11): p. 1078-1082.

21. Alford, P.W., et al., Vascular smooth muscle contractility depends on cell shape. Integr Biol (Camb), 2011. 3(11): p. 1063-70. 
22. Lin, S., M. Sandig, and K. Mequanint, Three-dimensional topography of synthetic scaffolds induces elastin synthesis by human coronary artery smooth muscle cells. Tissue engineering. Part A, 2011. 17(11-12): p. 1561-71.

23. Thery, M., Micropatterning as a tool to decipher cell morphogenesis and functions. J Cell Sci, 2010. 123(Pt 24): p. 4201-13.

24. Bettinger, C.J., R. Langer, and J.T. Borenstein, Engineering substrate topography at the micro- and nanoscale to control cell function. Angewandte Chemie, 2009. 48(30): p. 5406-15.

25. King-Chuen, W., et al., Nanotechnology in the regulation of stem cell behavior. Science and Technology of Advanced Materials, 2013.14(5): p. 054401.

26. Liu, W.F., Mechanical regulation of cellular phenotype: implications for vascular tissue regeneration. Cardiovascular research, 2012. 95(2): p. 215-22.

27. Wozniak, M.A., et al., Focal adhesion regulation of cell behavior. Biochim Biophys Acta, 2004. 1692(2-3): p. 103-19.

28. Chang, S., et al., Phenotypic modulation of primary vascular smooth muscle cells by short-term culture on micropatterned substrate. PLoS One, 2014. 9(2): p. e88089.

29. Dalby, M.J., N. Gadegaard, and R.O. Oreffo, Harnessing nanotopography and integrinmatrix interactions to influence stem cell fate. Nat Mater, 2014. 13(6): p. 558-69.

30. Lopez, J.I., J.K. Mouw, and V.M. Weaver, Biomechanical regulation of cell orientation and fate. Oncogene, 2008. 27(55): p. 6981-93.

31. Bialecki, R.A., T.J. Kulik, and W.S. Colucci, Stretching increases calcium influx and efflux in cultured pulmonary arterial smooth muscle cells. Am J Physiol, 1992. 263(5 Pt 1): p. L602-6.

32. Matchkov, V.V., O. Kudryavtseva, and C. Aalkjaer, Intracellular $\mathrm{Ca}(2)(+)$ signalling and phenotype of vascular smooth muscle cells. Basic Clin Pharmacol Toxicol, 2012. 110(1): p. $42-8$.

33. Rowlands, A.S. and J.J. Cooper-White, Directing phenotype of vascular smooth muscle cells using electrically stimulated conducting polymer. Biomaterials, 2008. 29(34): p. 4510-4520.

34. Heidkamp, M.C. and B. Russell, Calcium not strain regulates localization of alphamyosin heavy chain mRNA in oriented cardiac myocytes. Cell Tissue Res, 2001. 305(1): p. 121-7.

35. Wamhoff, B.R., et al., L-type Voltage-Gated Ca2+ Channels Modulate Expression of Smooth Muscle Differentiation Marker Genes via a Rho Kinase/Myocardin/SRFDependent Mechanism. Circulation Research, 2004. 95(4): p. 406-414.

36. Gusev, N.B., Some properties of caldesmon and calponin and the participation of these proteins in regulation of smooth muscle contraction and cytoskeleton formation. Biochemistry (Mosc), 2001. 66(10): p. 1112-21.

37. Mirensky, T.L., et al., Tissue-engineered vascular grafts: does cell seeding matter? Journal of pediatric surgery, 2010. 45(6): p. 1299-305.

38. Beamish, J.A., et al., Molecular regulation of contractile smooth muscle cell phenotype: implications for vascular tissue engineering. Tissue Eng Part B Rev, 2010. 16(5): p. 467-91.

39. Huber, A. and S.F. Badylak, Phenotypic changes in cultured smooth muscle cells: limitation or opportunity for tissue engineering of hollow organs? Journal of tissue engineering and regenerative medicine, 2012. 6(7): p. 505-11.

40. Campbell, J.H. and G.R. Campbell, Smooth muscle phenotypic modulation--a personal experience. Arteriosclerosis, thrombosis, and vascular biology, 2012. 32(8): p. 1784-9. 
41. Birukov, K.G., et al., Stretch affects phenotype and proliferation of vascular smooth muscle cells. Molecular and cellular biochemistry, 1995. 144(2): p. 131-9.

42. Chamley-Campbell, J.H. and G.R. Campbell, What controls smooth muscle phenotype? Atherosclerosis, 1981. 40(3-4): p. 347-357.

43. Chan-Park, M.B., et al., Biomimetic control of vascular smooth muscle cell morphology and phenotype for functional tissue-engineered small-diameter blood vessels. Journal of biomedical materials research. Part A, 2009. 88(4): p. 1104-21. 
SMCs Phenotype Modulation by Electro-responsive Hydrogel 


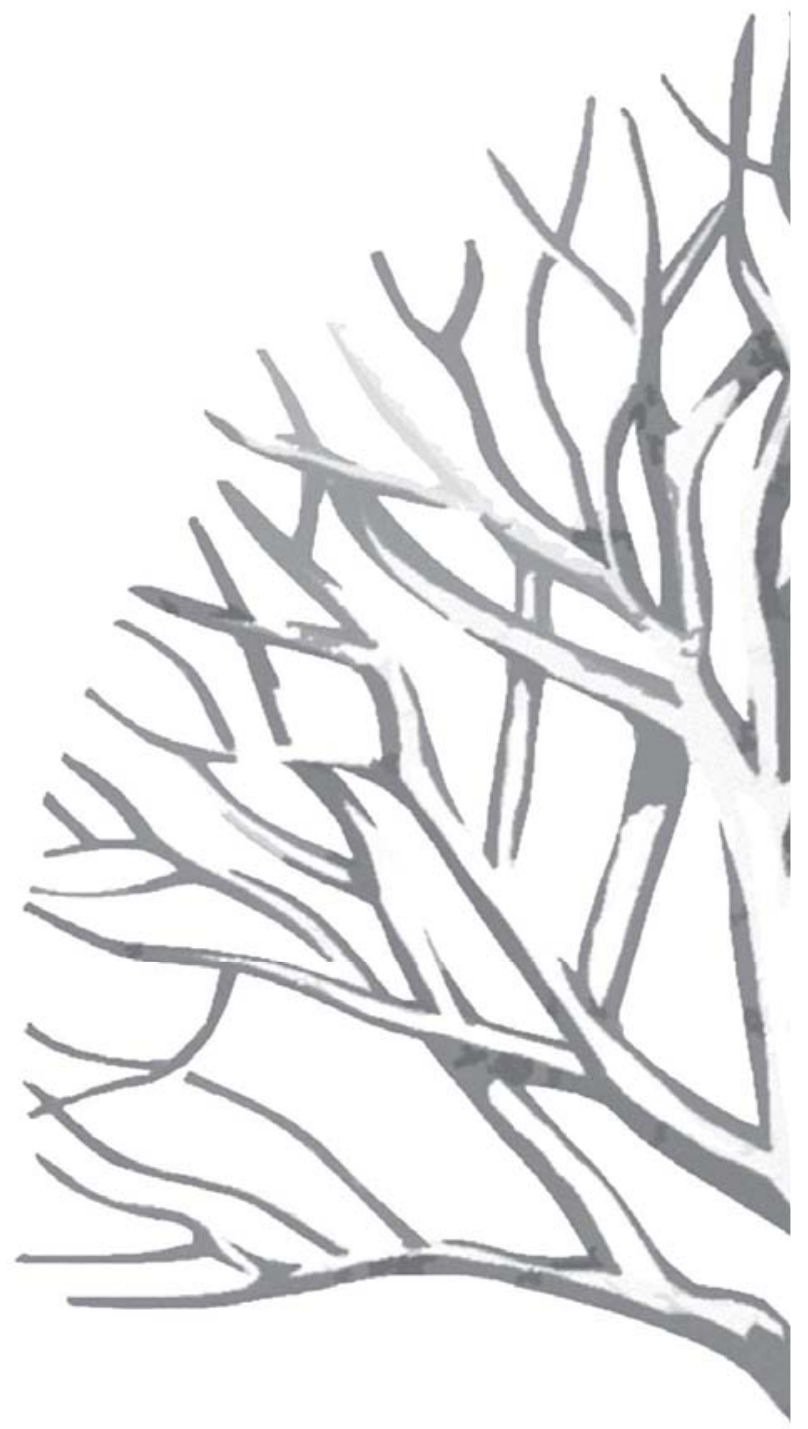




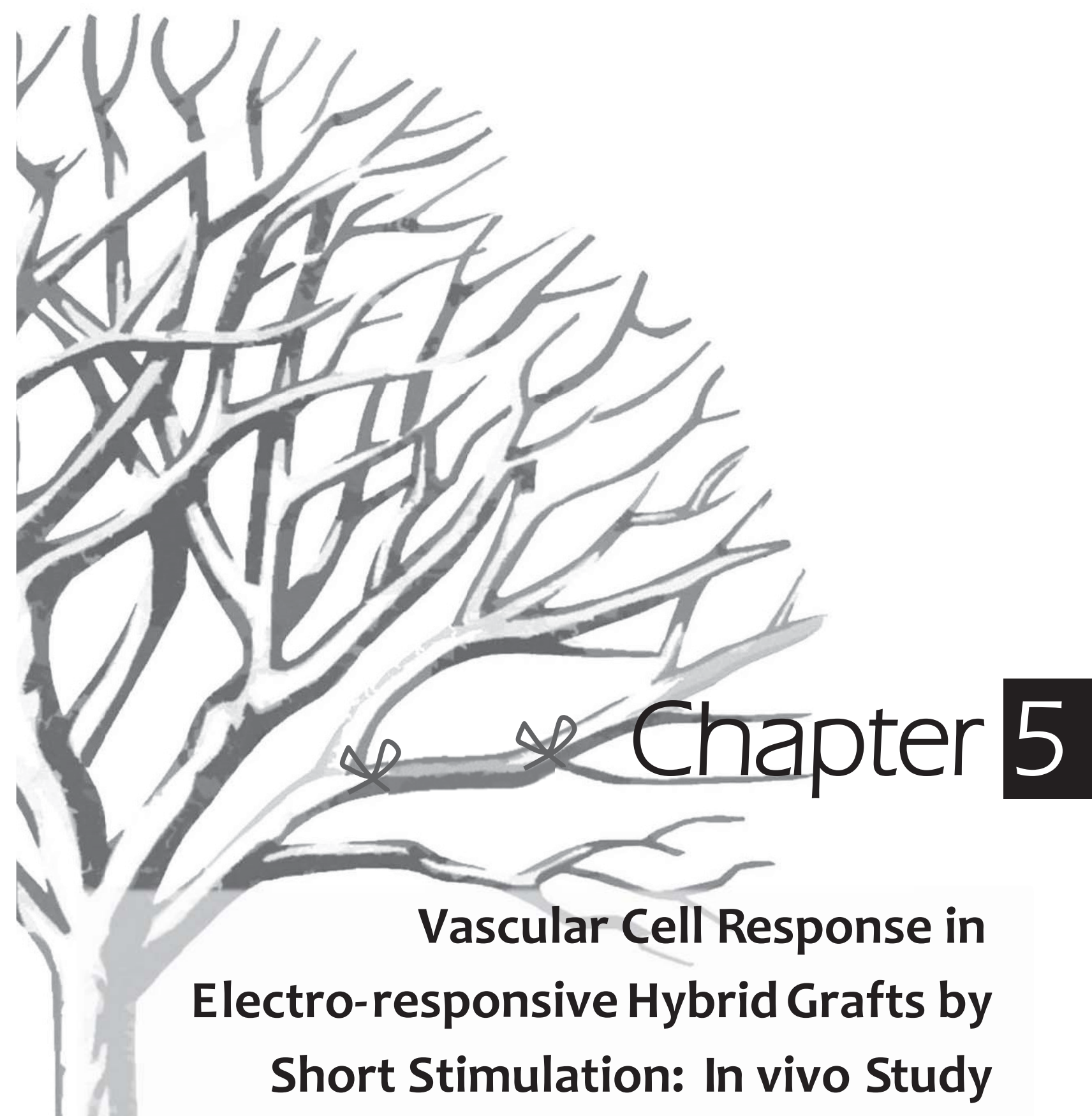

Nastaran Rahimi Allard Wagenaar Hanneke Cobelens Viviane Heijnen Daniel G. Molin Mark J. Post 


\section{Abstract}

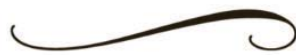

Directing cell growth and tissue formation are great challenges for successful development of tissue engineered vascular graft. When implanting bare scaffolds, the response of the recipient plays an important role in remodeling and maturation of the vessel substitute. In this study we evaluated the effect of external stimulation to facilitate and improve the functionality of a smalldiameter tissue engineered graft in vivo. Electro-spun PCL grafts were coated and functionalized by electro-responsive Polyacrylic acid (PAA)/fibrin hydrogel. Implanted grafts were subjected to an alternating electrical field $(1 \mathrm{~mW} / \mathrm{cm} 2$, $0.0167 \mathrm{~Hz}$ ) in ventro-dorsal axis (1 hour) followed by 1 hour in bi-lateral axis, once per week. Morphometric analysis of explanted grafts showed enhanced coverage of endothelium as well as inhibition of neo-intima formation in the stimulated PAA/PCL grafts compared with non-stimulated PCL and PAA/PCL grafts. Additionally, infiltration of cells into the grafts was significantly increased by stimulation after 4 and 8 wks of implantation. The promising results of our set-up on improving cell migration and growth in vascular grafts suggest high potential of electro-responsive hydrogels applications in developing small-diameter vascular grafts. 


\section{Introduction}

Limitations with autologous vascular vessels in bypass surgery initiated development of tissue engineered grafts. In spite of successful clinical applications for large caliber blood vessel substitutes, the success rate for small diameter grafts is limited due to high thrombogenicity, intimal hyperplasia, aneurysm and compliance mismatch. The two major approaches in developing tissue engineered scaffold-based vascular grafts are pre-seeded and cultured grafts or bare scaffolds. Although successful clinical application of pre-seeded grafts has been reported, risks of immune reaction to autologous cells as well as difficulty in harvesting and seeding of cells from patients with limited cell sources raise concerns with timely availability of the grafts [1]. However, there are also challenges with bare scaffolds. For proper function they highly depend on in vivo maturation of the graft by tissue growth while the graft material itself is gradually degrading. To date, several studies have been performed on the functionality of biodegradable polymer grafts in vivo with promising results [2, 3]. Ideally, a scaffold needs to contain several biological and mechanical properties to perform well in the human vascular system. It should be biocompatible, non-thrombogenic and have sufficient mechanical strength to withstand blood pressure. Also it should provide a proper support for cells to infiltrate the structure and deposit extracellular matrix. Furthermore, the bare scaffold needs to provide appropriate signals to improve and optimize cell migration as well as tissue growth in the scaffold [4]. Stickler et al. have reported the effect of external mechanical forces to improve the development and growth of vascular grafts in the peritoneum in vivo [5]. In this study, pulsed mechanical stretch application resulted in greater collagen organization, F-actin expression and mechanical properties than with non-pulsed stress application [5].

We have previously shown the ability of Polyacrylic acid/Fibrin hydrogel to facilitate cell migration, fiber alignment and enhancing collagen production and remodeling in vitro by applying short electrical stimulation of 2 hrs per week $[6,7]$. In the current study we investigated patency and performance of a functionalized electro-spun PCL tubular structure coated with Polyacrylic acid hydrogel as an electro-responsive graft. Polycaprolactone $(P C L)$ is a frequently used biomaterial for vascular tissue engineering due to its biodegradability, good biocompatibility and excellent mechanical properties in its fibrous and 
electrospun form [8]. PAA/Fibrin coated PCL grafts were evaluated in a Rat aorta interposition model up to $8 \mathrm{wks}$ and the effect of external electrical stimulation on endothelization, cell infiltration and foreign body reaction were studied.

\section{Materials and Methods}

\section{Grafts preparation}

Monomer solution containing acrylic acid (99.9\% purity, Merck Germany), N-N'Methylenebisacrylamide (MBAA. 2 wt\% of monomer, Sigma, The Netherlands), 4,4'-Azobis(4-cyanovaleric acid)(ACVA, Sigma, The Netherlands), Fibrinogen (20mg/mL, Sigma, The Netherlands) and thrombin (20 IU/mL, Sigma, The Netherlands) was prepared and pipetted through 0.2 micron sterilized filters and subsequently degassed by bubbling Argon through the solution inside the hood. MBAA and ACVA were used as cross-linker and radical initiator respectively. Electrospun $\mathrm{PCL}$ grafts (Xeltis Company, Switzerland) were dressed onto a glass mandrel of $2.5 \mathrm{~mm}$ diameter, dip coated in the monomer solution, flushed by Argon and exposed to UV exposure for 8 minutes. The coating step was repeated 3 times to ensure adequate hydrogel coating. The glass mandrel was removed and prepared coated grafts were then washed with acetone and immersed in deionized water for $48 \mathrm{hrs}$ to remove residual components.

\section{In vivo implantation}

Experimental methods and procedures were approved by the laboratory animal ethics committee of Maastricht University. Twenty four male Sprague Dawley rats (weight range 300-350 gr) were obtained from Harlan. Animals were anesthetized with $0.4 \mathrm{~L} \mathrm{O}_{2}$ and $2.5 \%$ Isoflurane and prepared for surgery, including continuous temperature monitoring. The infrarenal abdominal aorta was exposed through a midline laparotomy under an operation microscope (Zeiss 3062 46). After clamping, a $1.5 \mathrm{~cm}$ piece of aorta was removed and a gamma sterilized graft was sutured end-to-end with Covidien Monosof 10-0 N2540 suture. The distal clamp and proximal clamp (10 s later) were then released to restore blood flow. After closure, the rats were recovered. Animals were kept in groups of at least 2 rats in standard cages with free access to 
normal food and water maintained at $22^{\circ} \mathrm{C}$. Rats were followed up for 4 and 8 wks after which grafts were explanted.

\section{Electrical stimulation of implanted grafts}

The graft recipients were randomly divided in two groups of sham-stimulated $(n=16)$ and electrically stimulated $(n=8)$. In order to stimulate the implanted grafts, rats were exposed to electrical stimulation on day 2, 9 and 16 day of implantation as follows.

Animals were anesthetized $1.5-2 \%$ isoflurane in $0.4 \mathrm{~L} / \mathrm{min} \mathrm{O}_{2}$. Abdomen, back and both flanks of rats were shaved and cleaned to be prepared. Conductive Redux ${ }^{\circledR}$ Gel (Hewlett Packard, Andover, MA) was applied to the skin to reduce skin resistance. The in vivo setting of electrical stimulation was adopted from our previous studies and verified with peri-aortic voltage and current measurements.

To electrically stimulate the implanted grafts, rats were exposed to electrical field $(1 \mathrm{~mW} / \mathrm{cm} 2,0.0167 \mathrm{~Hz}$ ) by placing ECG electrodes (IDEE, Maastricht University) in sagittal axis for 1 hour, followed by 1 hour frontal axis stimulation. The polarity of voltage was changed every $60 \mathrm{sec}$ by a programmable switch. At the end of the 2 hours, the stimulation sites were cleaned with alcohol and animals were transferred to individual cages and after recovery returned to the housing facility. Non-stimulated PCL and PAA coated PCL grafts served as controls. Animals were sacrificed either after 4 or 8 wks.

\section{Immunohistochemistry and morphometric analysis}

Grafts were explanted after 4 and 8 wks of implantation, fixed in $4 \%$ Paraformaldehyde (PFA), cut longitudinally in two halves and embedded in paraffin. Paraffin-embedded grafts were cut into $5 \mu \mathrm{m}$-thick longitudinal sections processed for histology. Tissue sections were stained for Hematoxyline and Eosin (Eosin Y Solution 0.2\%, J.T.Baker, Hematoxylin solution according to Mayer, Sigma) to evaluate cell infiltration into the graft. For immunohistochemistry, sections were blocked by immersing in 10\% bovine serum albumin for $1 \mathrm{hr}$ at room temperature. Samples were then incubated with primary antibodies: monoclonal anti-CD31 (BD Company) for enthothelialization, anti-CD68 (Bio-connect) for macrophage detection and collagen II (Bio-connect) for enchondral bone formation as a precursor of calcification. 
Endothelial coverage was measured as the length of the Endothelial cell layer and expressed as percentage of the total graft length. Intima hyperplasia coverage and average thickness of neo-intima was measured by defining the distance between the Endothelial layer and luminal edge of the graft. To evaluate cell infiltration and growth, the number of elongated cells present in graft structure were counted and presented as cell density.

\section{Ultrasound and angiography}

Rats were anesthetized before performing the ultrasound and angiography measurements as described earlier. Graft patency was evaluated by postimplantation analysis by ultrasound and pre-euthanasia angiography. Ultrasound was performed along the longitudinal and transversal axis by using the Visual sonic machine (VEVO 2100) on day 9 and 16 after implantation to evaluate lumen diameters. Obtained images were processed by VEVO 210015.0 software.

Angiography was assessed just prior to euthanasia by injection of $3 \mathrm{ml}$ contrast agent (Hexabrix ${ }^{\circledR}$, Guerbet Netherlands BV) through a catheter into the carotid artery. Lumens of grafts were monitored (Pulsera, Philips, the Netherlands) to evaluate aneurysm or stenosis. After Angiography, rats were euthanized and grafts were explanted.

\section{Statistical analysis}

Data were expressed as mean+SD. Statistical analysis of the results was performed by student $t$ test and, when appropriate two-way measurement ANOVA (Graph pad prism 4) to evaluate significant effect of graft material type and implantation time and compare groups mean with Bonferroni post-hoc test. $P$ value less than 0.05 was considered to be significant in all the analyses. 


\section{Results}

\section{Grafts implantation and patency}

PCL and PAA coated PCL grafts were implanted as abdominal artery interposition in 24 male rats. Grafts showed good suturability and implantation surgery was performed effectively. No blood leakage was observed upon releasing the clamps and during preclotting of the graft (Fig.1 B-E). The electrical stimulation was carried out successfully during anesthesia (Fig.1 F,G). The patency of grafts was determined by arterial Doppler ultrasound and Angiography analysis. (Fig.2). Four out of 7 PCL, 7 out of 7 non-stimulated $\mathrm{PAA} / \mathrm{PCL}$ and 4 out of 4 stimulated $\mathrm{PAA} / \mathrm{PCL}$ grafts remained patent during implantation of 4 wks (N.S. P value: 0.06). Grafts showed all 100\% open lumen during 8 wks implantation. Aneurysms were found in 3 of 12 grafts in the nonstimulated PCL control group, in 3 out of 12 non-stimulated PAA/PCL grafts and $0 / 12$ in stimulated PAA/PCL grafts.
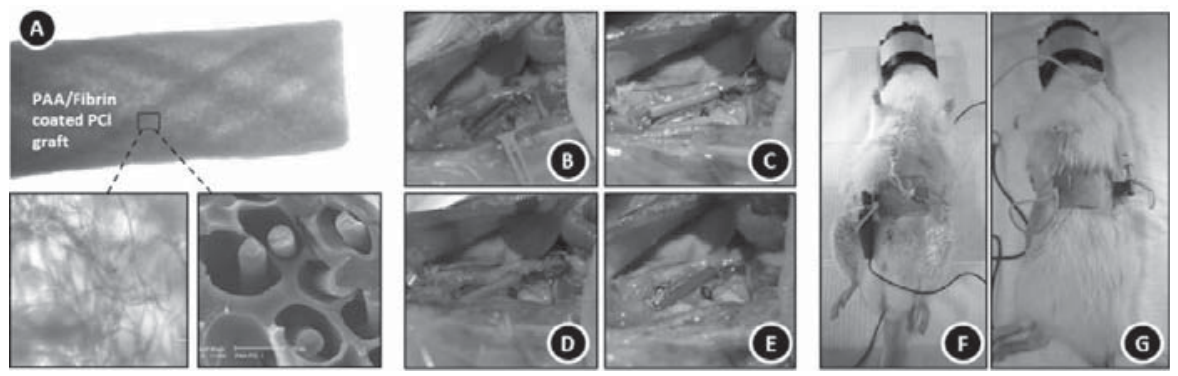

Fig 1. Light and scanning electron microscopy of Poly acrylic acid/Fibrin coated electrospun Polycaprolactone vascular grafts (A). Grafts were implanted as an interposition aorta in rats. The aorta was clamped and dissected (B), the graft was sutured as an end-to-end graft into the proximal (C) and distal aorta (D). The clamps were then removed to allow blood flow into the graft (E). Rats in stimulated groups were subjected to an electrical field of $1 \mathrm{~mW} / \mathrm{cm} 2,0.0167 \mathrm{~Hz}$ in ventro-dorsal (1 hr) (F) and bi-lateral (1 hr) axis (G) on days 2, 9 and 16 of implantation.

\section{Neo-intima formation}

Neointima primarily consisted of Smooth muscle cells (SMCs) forming multiple layers of cells between the endothelium and the lumen side of the graft [9]. At 4 wks follow up, PAA coating of PCL resulted in more neointima formation that 
was reduced again to the uncoated level upon electrical stimulation (Fig. $3 A, B, C, G)$. The thickness of neointima in stimulated $P A A / P C L$ grafts was comparable with uncoated $P C L$ grafts $(P>0.05)$. By discontinuing stimulation after 4 wks, neo- intima was 4.8 -fold increased at 8 wks follow up to the same level of non-stimulated PAA/PCL grafts (Fig.3D, G, P<0.01). Nonstimulated $P C L$ grafts showed 3 -folds increase in neointima thickness from 4 to 8 wks follow up but still remained significantly lower than stimulated and non-stimulated PAA/PCL grafts at 8 wks (Fig.3F, G, P<0.01). In non-stimulated $\mathrm{PAA} / \mathrm{PCL}$ neointima formation was the same at $4 \mathrm{wks}$ and 8 wks follow up. Using two-way anova analysis our results showed implantation time ( $F$ test $p$ value <0.0001) and graft material type ( $F$ test $p$ value $<0.0001)$ are significant factors to regulate Neo-intima formation.
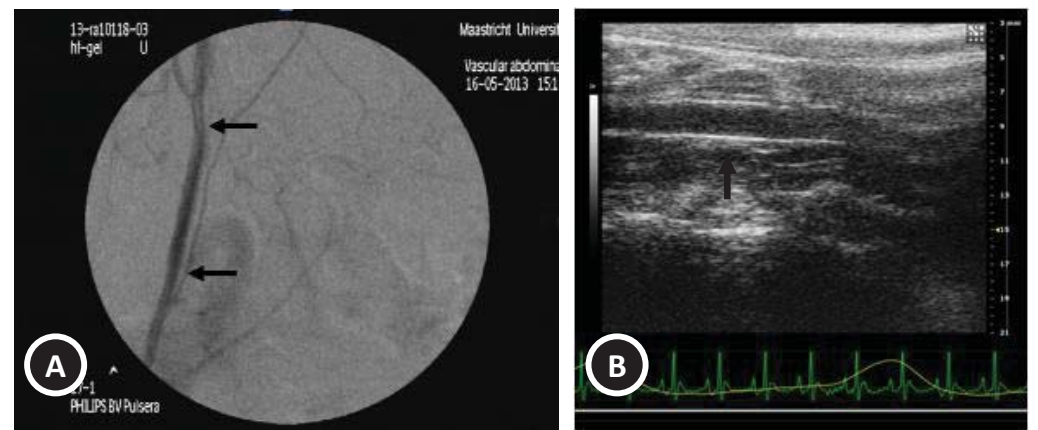

\begin{tabular}{|c|c|c|c|c|c|c|}
\hline \multirow[b]{2}{*}{ Implantation time (wks) } & \multicolumn{2}{|c|}{$\begin{array}{l}\text { PAA/PCL } \\
\text { Stimulated }\end{array}$} & \multicolumn{2}{|c|}{$\begin{array}{c}\text { PAA/PCL } \\
\text { Non-stimulated }\end{array}$} & \multicolumn{2}{|c|}{$\begin{array}{c}\text { PCL } \\
\text { Non-stimulated }\end{array}$} \\
\hline & 4 & 8 & 4 & 8 & 4 & 8 \\
\hline No. of grafts implanted & 4 & 5 & 7 & 5 & 7 & 5 \\
\hline No. of grafts failed (closed lumen) & 0 & 0 & 0 & 0 & 3 & 0 \\
\hline Aneurysm & 0 & 0 & 0 & 3 & 3 & 0 \\
\hline Survival rate \% & 100 & 100 & 100 & 100 & 57 & 100 \\
\hline
\end{tabular}

Fig.2. Angiography ( $28^{\text {th }}$ day) (A) and ultrasound (16 th day) (B) images of PAA/PCL grafts showing open lumen with no stenosis or aneurysm. Arrows indicate the anastomoses sites of the graft. The patency of the grafts was evaluated after 4 and 8 wks of implantation (C). 


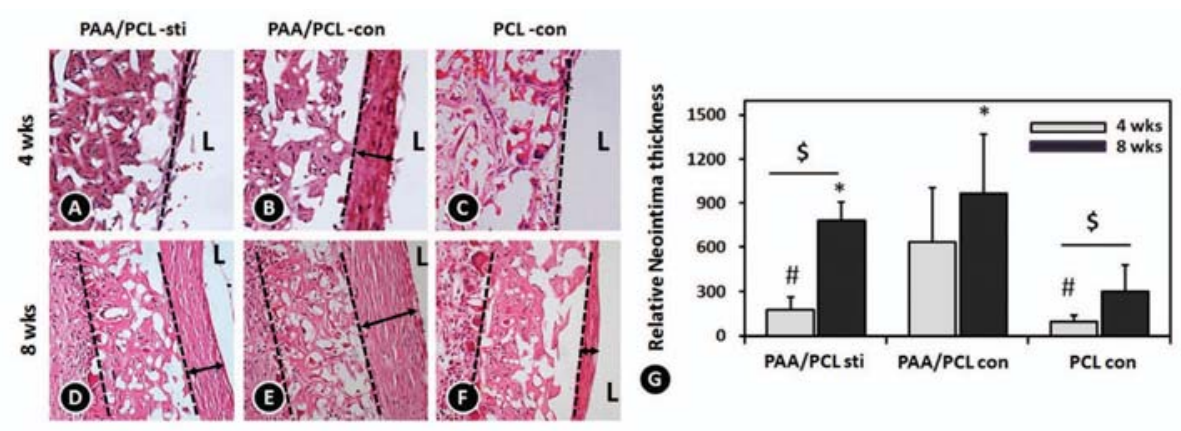

Fig.3. Histological analysis of explanted grafts section by Hematoxylene/Eosin staining showing neointima formation in stimulated-PAA/PCL grafts $(A, D), P A A / P C L(B, E)$ and $P C L$ grafts $(C, F)$ in non-stimulated control condition after 4 and 8 wks of implantation. Neointima formation was quantified by measuring the thickness of developed cell layers between endothelium and the luminal edge of the grafts. Notice the sharp demarcation between graft and neointima in B, D, E and F. Pound and asterisks and signs indicate $\mathrm{p}<0.05$ compared with PAA/PCL con at $4 \mathrm{wks}$ and PCL con at $8 \mathrm{wks}$, respectively. Dollar sign indicate $p<0.05$ for underlined groups.

\section{Endothelization}

Grafts were evaluated for coverage of Endothelial cells by $C_{3} 1$ staining on longitudinal sections (Fig.4). At 4 wks, PCL grafts showed very limited coverage of Endothelial cells only in the area closed to the anastomosis sites (33\%) suggesting growth of Endothelium from the adjacent aorta fragments [10]. Non-stimulated PAA/PCL grafts also showed limited Endothelization (31\%). Electrical stimulation of the $P A A / P C L$ grafts resulted in significantly higher coverage of Endothelium (60\%) over the length of graft including the central part of the graft. At $8 \mathrm{wks}$, endothelialization was nearly complete with $85 \%$ coverage and no differences between the groups ( $F$ test $p$ value $>0.0001$ for Implantation time).

\section{Cell ingrowth}

On hematoxyline/eosin stained sections, the number of elongated nuclei was determined to define infiltration by myofibroblasts or SMCs in the graft wall (Fig. 5). At 4 wks follow up, cell density was highest in stimulated PAA/PCL grafts after 4 wks of implantation (Fig. 5 A). Non-stimulated PAA/PCL and PCL 
graft showed significantly lower cell ingrowth compared to stimulated samples (Fig.5 B,C,D) (F test $P$ value <0.0001 for graft material type).
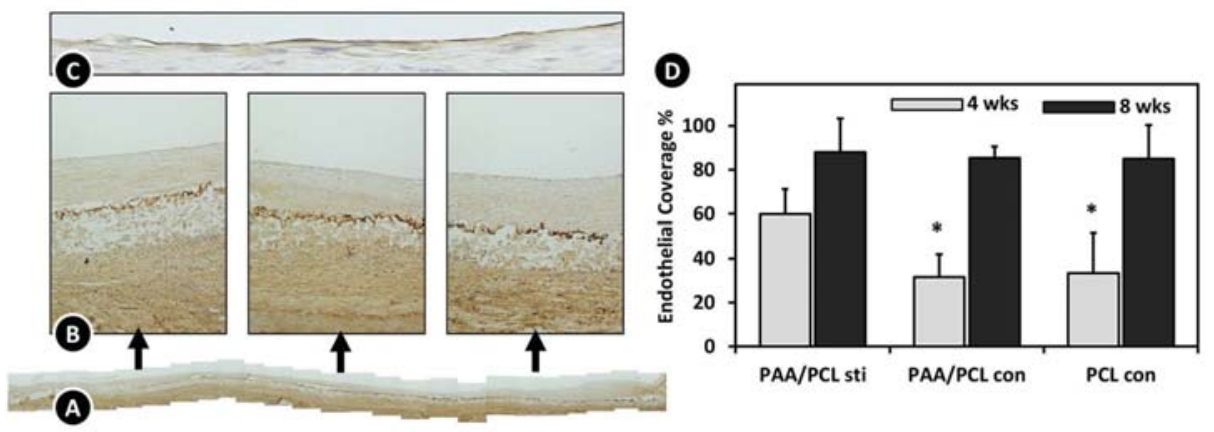

Fig.4. Histological images of PAA/PCL grafts in control condition by $2.5 \times(a), 10 x(B)$ and 40 $x(C)$ magnification after 8 wks of implantation stained by $C D 31$ antibody represents high coverage of endothelium in the length of the graft. The coverage of Endothelial layer was quantified as percentage of $\mathrm{CD}_{3} 1$ positive length to the total length of graft. Asterisks indicates $\mathrm{p}<0.05$ compared to PAA/PCL sti at $4 \mathrm{wks}$.

The distribution was also different: In stimulated PAA/PCL grafts cells were distributed throughout the thickness of the graft, while in non-stimulated control grafts cells were mainly located at the adventitia side of the scaffold. The pattern was the same at 8 wks follow up and cell density levels had only slightly increased in both unstimulated grafts ( $F$ test $P$ value $>0.5$ for implantation time). Our observation indicates that $\mathrm{PAA} / \mathrm{PCL}$ grafts provide an appropriate substrate to support cell ingrowth which is further enhanced by electrical stimulation.

\section{Foreign body reaction}

Using CD68 immunostaining for visualizing infiltration of macrophages (Fig. 6 A-F), the level of foreign body reaction (FBR) was quantified (Fig. 6G) in the graft and implantation area [11]. Macrophages are a crucial part of the inflammatory process and they also play an important role in remodeling of the grafts through phagocytosis and secretion of cytokines such as VEGF [12]. 

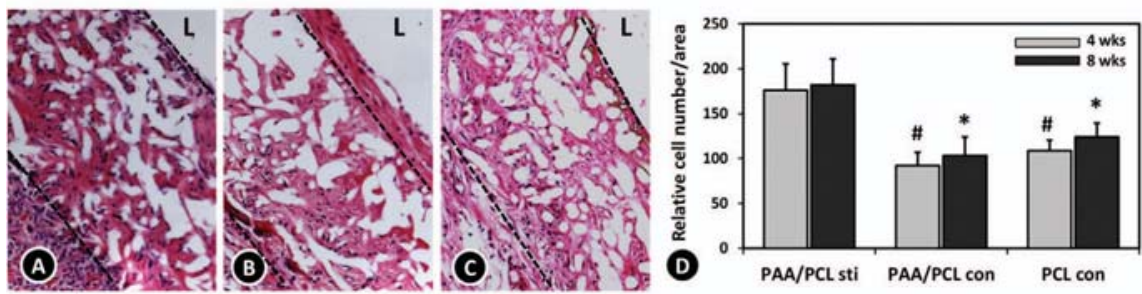

Fig.5. Histological images of stimulated PAA/PCL (A) and non-stimulated control PAA/PCL (B) and PCL (C) grafts represents cellular infiltration into the graft wall after 4 wks of implantation. Cellular density was quantified by counting the elongated cells in the grafts after 4 and 8 wks of implantation (D). Pound and Asterisks signs indicate $p<0.05$ compared to $\mathrm{PAA} / \mathrm{PCL}$ sti at $4 \mathrm{wks}$ and $8 \mathrm{wks}$, respectively.

In the FBR to biomaterials, macrophages take on a giant cell phenotype while encapsulating the material. All PAA/PCL and PCL grafts studied after 4 and 8 wks implantation contained giant cells with significant effects of graft material type ( $F$ test $p$ value $<0.02$ ) and implantation time ( $F$ test $p$ value $<0.001$ ). In the areas surrounding the graft the FBR was substantially less than within the graft. Electrically stimulated PAA/PCL grafts showed more even dispersion of macrophages through the wall thickness compared to non-stimulated grafts where the macrophages were mainly located at the adventitial side of the scaffold. After 4 and 8 wks follow up, PAA/PCL grafts exposed to stimulation showed significantly less inflammatory response compared to $\mathrm{PCL}$ alone. Although the distribution of CD68 immunopositivity in PAA/PCL grafts was affected by stimulation, the total level of FBR was not different between the stimulated and control condition. The FBR at the adventitia side in PAA/PCL grafts in both control and stimulated condition was not significantly different from PCL after 4 wks. At 8 wks follow up however, the FBR at the adventitia side of PCL grafts was significantly increased compared to 4 wks and PAA/PCL grafts.

\section{Calcification}

Vascular calcification is defined as deposition of calcium components either in intimal or medial part of the graft depending on the stimulated molecular pathway. The calcification process is initiated by differentiation of SMCs via an enchondral pathway as indicated by expression of collagen II [13]. Deposition 
of collagen II was therefore taken as a surrogate marker for a precalcified lesion [14]. At 8 wks follow up, grafts displayed collagen II expression confined to the neointima. To quantitatively compare collagen II deposition between the groups, images were evaluated by measuring Collagen II positive area normalized to the neointima area. Samples showed an average of $10 \pm 5 \%$ coverage of collagen II deposition with no significant difference between groups.
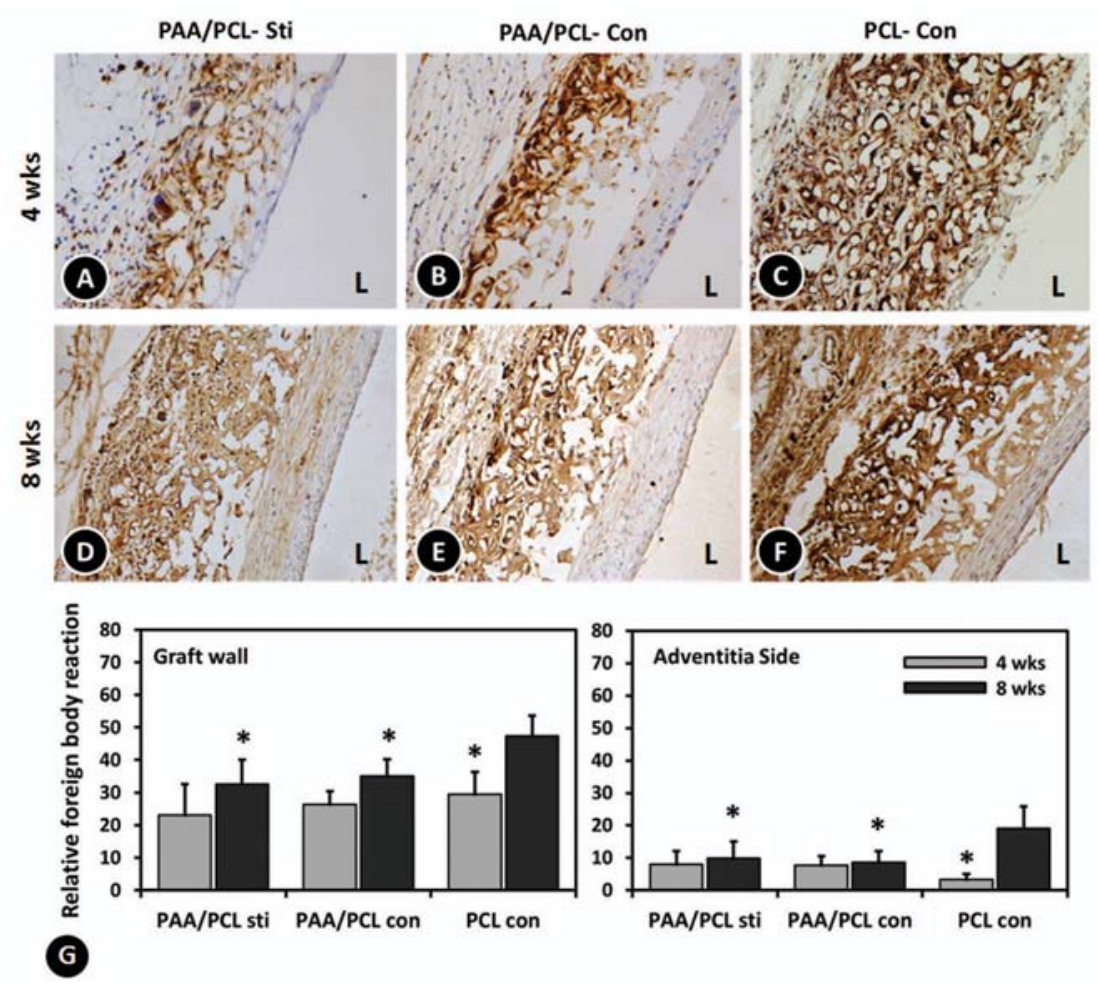

Fig.6. Immunohistological images of stimulated PAA/PCL (A, D) and non-stimulated control PAA/PCL (B, E) and PCL (C, F) grafts stained by CD68 antibody determine the infiltration of macrophages at 4 and 8 wks follow up. The Foreign body reaction was evaluated in the graft wall and the surrounding tissue on the adventitia side (G). Asterisk indicates $\mathrm{P}$ value $<0.05$ compared to $\mathrm{PCL}$ con at $8 \mathrm{wks}$. 

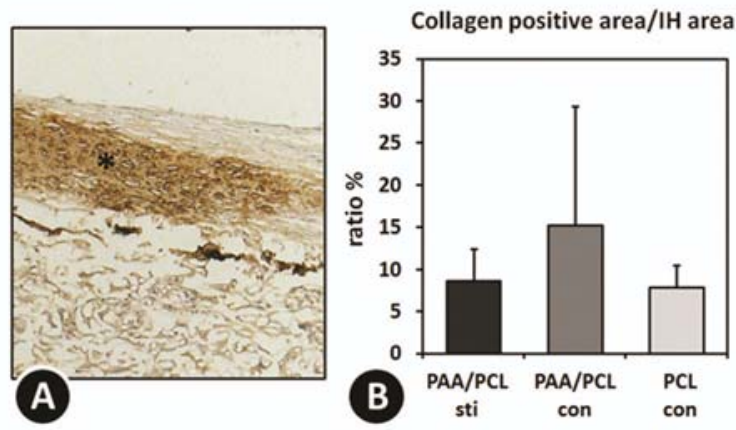

Fig.7. Collagen II immunostaining of PAA/PCL graft implanted for 8 wks under control condition (A) Asterisk (*) indicates the collagen II positive area. Calcification in stimulated $\mathrm{PAA} / \mathrm{PCL}$ and non-stimulated control $\mathrm{PAA} / \mathrm{PCL}$ and $\mathrm{PCL}$ grafts were quantified by evaluating the collagen II positive area normalized to the neointima area.

\section{Discussion}

In this study we presented a new method to stimulate vascular graft development in vivo. To our knowledge this is the first time external forces are applied to facilitate tissue growth for implanted tissue engineered grafts. Electrospun polycaprolactone grafts were functionalized into an electroresponsive hybrid structure by incorporating cross-linked polyacrylic acid into the structure. Polyacrylic acid coating provides a smooth lumen surface of the graft and alters the surface charge of the graft towards a more negative charge. In addition, PAA hydrogel has the ability to respond to environmental stimuli by association and dissociation of its carboxylic groups $[15,16]$. In our study, PAA coated PCL grafts showed more neointima compared to bare PCL grafts. However, electrical stimulation effectively inhibited the growth of neointima formation in PAA/PCL grafts at $4 \mathrm{wks}$ of implantation. When an acellular vascular graft is implanted, a series of complicated events occurs [17]. Neointima is mostly built up by migration of host SMCs towards the graft lumen side, forming a neointima [18]. Recent studies also reported the possible incorporation of SMCs with synthetic phenotype derived from the bloodstream to form neo-intima [19]. SMCs in the medial layer of native vessels show a contractile phenotype with high expression of a-actin and SMMHC proteins and very low proliferation rate [20, 21]. In neointima formation however, SMCs are typically in a synthetic phase. Injury of the vascular wall could activate 
SMCs proliferation by suppression the inhibitory factors of SMCs proliferation such as heparin sulfate and NO [22, 23]. Damaged SMCs express basic fibroblast growth factor (FGF-2), which further stimulates SMC proliferation [24]. One possible explanation could be the alteration of surface roughness in Acrylic acid hydrogel coated grafts compared to bare PCL ones. Surface roughness is an important characteristic of grafts determining platelet adhesion and interactions between blood cells and the scaffold [25]. Activated platelets play an important role in SMCs migration and proliferation and therefore thickening of the neointima [20]. The mechanism for the inhibitory effect of electrical stimulation on neointima formation remains speculative at this point. In a separate study we show differentiation of SMCs towards a contractile phenotype by electrical stimulation (submitted for publication).

Our observation also suggests an active role of acrylic acid/Fibrin hydrogel in attracting Endothelial cells and SMCs into the graft. Improved coverage of the graft by Endothelial cells in electrically stimulated samples may have contributed to quiescence of neointima formation. Based on previous in vitro studies on the cellular effects of electrical stimulation of PAA/PCL graft it is likely that a conformational change of the PAA/PCL grafts is responsible of reduced neointima formation and re-endothelialization, although the in vivo mechanisms are not completely understood yet. Applying an electrical field creates a negatively charged surface in the graft by dissociating carboxylic groups into - $\mathrm{COO}$. In addition, polyacrylic acid hydrogels have shown negative zeta potential even when the structure is electrically neutral [26]. In spite of the negative charge, Chen et al. showed adhesion and growth of Endothelial cells on polyacrylic substrates to subconfluent levels $[26,27]$. The gel adsorbs proteins quite efficiently and these proteins likely act as a peptide bridge between the surface and cells [26]. Adhesive proteins could also regulate migration and proliferation of Endothelial cells. Albumin, fibrinogen and IgGs are present in the bloodstream and could be adsorbed to the polymer surface as shown in an in vivo model by Nojiri et. al [28]. Hence, the acrylic acid coated $\mathrm{PCL}$ graft has the affinity to adsorb proteins, which could regulate cellular behavior upon electrical stimulation.

Electrically activated polyacrylic acid could also provide a gradient in ion transport by induced changes in osmotic pressure in the graft wall and the surrounding tissue, which might affect cell migration. In addition, structural changes of the hydrogel (swelling/shrinkage) when exposed to electrical field could also enhance infiltration of cells into the structure as was tested in vitro 
[6]. In addition to a higher density of elongated cells in the stimulated grafts compared to controls, more even distribution of macrophages identified by CD68 staining also confirms the functionality of our proposed system to affect cell infiltration. Macrophages play a critical role in remodeling of tissue engineered vascular grafts by secretion of a multitude of cytokines and chemokines [12, 29]. A recent study by Spiller et al. reported the role of different macrophage phenotypes regulating angiogenesis and scaffold vascularization by secretion of Vascular Endothelial growth factor (VEGF) and platelet derived growth factor-BB (PDGF-BB) [30].

Our proposed system showed significant enhancement in cell growth and endothelialization and reduced neointima formation as a result of electrical stimulation. With this study we provided proof of concept for applying external stimuli in order to induce desirable cell behavior by conformational changes of the graft structure. Electro-responsive hydrogels can be clinically implemented to facilitate vascular graft maturation in patients by temporarily imposing an external electrical field on the site of implantation.

\section{Acknowledgment}

This research forms part of the research program of the BioMedical Materials institute, co-funded by the Dutch Ministry of Economic Affairs. The financial contribution of the Nederlandse Hartstichting is gratefully acknowledged. D.G.M. is supported by the Euregio meuse-Rhine Interreg IV-A BioMIMedics project. We thank Xeltis Company (Switzerland) for providing electro-spun PCL grafts. We also thank Rafel Dera and Frederik Horeman from Hasselt University, Belgium and Mohammad Ghomi Rostami from Sharif University of Technology, Tehran for their support and assistance.

\section{Author Disclosure Statement}

No competing financial interests exist. 


\section{References}

1. Nerem, R.M. and D. Seliktar, Vascular tissue engineering. Annual review of biomedical engineering, 2001. 3: p. 225-43.

2. Soletti, L., et al., In vivo performance of a phospholipid-coated bioerodable elastomeric graft for small-diameter vascular applications. Journal of biomedical materials research. Part A, 2011. 96(2): p. 436-48.

3. Wise, S.G., et al., A multilayered synthetic human elastin/polycaprolactone hybrid vascular graft with tailored mechanical properties. Acta biomaterialia, 2011. 7(1): p. 295-303.

4. Cho, S.W., et al., Enhancement of in vivo endothelialization of tissue-engineered vascular grafts by granulocyte colony-stimulating factor. Journal of biomedical materials research. Part A, 2006. 76(2): p. 252-63.

5. Stickler, P., et al., Cyclically stretching developing tissue in vivo enhances mechanical strength and organization of vascular grafts. Acta biomaterialia, 2010. 6(7): p. 244856.

6. Rahimi, N., et al., Electrosensitive polyacrylic acid/fibrin hydrogel facilitates cell seeding and alignment. Biomacromolecules, 2012. 13(5): p. 1448-57.

7. Rahimi, N., et al., Short Stimulation of Electro-Responsive PAA/Fibrin Hydrogel Induces Collagen Production. Tissue engineering. Part C, Methods, 2014.

8. de Valence, S., et al., Long term performance of polycaprolactone vascular grafts in a rat abdominal aorta replacement model. Biomaterials, 2012. 33(1): p. 38-47.

9. Desai, M., et al., Inhibition of neointimal formation and hyperplasia in vein grafts by external stent/sheath. Vasc Med, 2010. 15(4): p. 287-97.

10. Zheng, W., et al., Endothelialization and patency of RGD-functionalized vascular grafts in a rabbit carotid artery model. Biomaterials, 2012. 33(10): p. 2880-91.

11. Badylak, S.F. and T.W. Gilbert, Immune response to biologic scaffold materials. Semin Immunol, 2008. 20(2): p. 109-16.

12. Roh, J.D., et al., Tissue-engineered vascular grafts transform into mature blood vessels via an inflammation-mediated process of vascular remodeling. Proceedings of the National Academy of Sciences, 2010. 107(10): p. 4669-4674.

13. Hruska, K.A., Vascular smooth muscle cells in the pathogenesis of vascular calcification. Circ Res, 2009. 104(6): p. 710-1.

14. Neven, E., et al., Chondrocyte rather than osteoblast conversion of vascular cells underlies medial calcification in uremic rats. Arterioscler Thromb Vasc Biol, 2010. 30(9): p. 1741-50.

15. Bawa, P., et al., Stimuli-responsive polymers and their applications in drug delivery. Biomed Mater, 2009. 4(2): p. 022001.

16. Qiu, Y. and K. Park, Environment-sensitive hydrogels for drug delivery. Advanced Drug Delivery Reviews, 2001. 53(3): p. 321-339.

17. Anderson, J.M., BIOLOGICAL RESPONSES TO MATERIALS. Annual Review of Materials Research, 2001. 31(1): p. 81-110.

18. Quint, C., et al., Decellularized tissue-engineered blood vessel as an arterial conduit. Proc Natl Acad Sci U S A, 2011. 108(22): p. 9214-9.

19. Iwata, H. and M. Sata, Origin of Cells That Contribute to Neointima Growth. Circulation, 2008. 117(24): p. 3060-3061. 
20. Louis, S.F. and P. Zahradka, Vascular smooth muscle cell motility: From migration to invasion. Exp Clin Cardiol, 2010. 15(4): p. e75-85.

21. Greif, D.M., Chapter 1: Vascular Embryology and Angiogenesis, Tunica Media: Smooth Muscle and Extracellular Matrix, in Vascular Medicine: A Companion to Braunwald's Heart Disease, J.A.B. Mark A. Creager, and Joseph Loscalzo, Editor. 2013, Elsevier.

22. Rzucidlo, E.M., K.A. Martin, and R.J. Powell, Regulation of vascular smooth muscle cell differentiation. Journal of Vascular Surgery, 2007. 45(6, Supplement): p. A25-A32.

23. Alef, M.J., E. Tzeng, and B.S. Zuckerbraun, Nitric oxide and nitrite-based therapeutic opportunities in intimal hyperplasia. Nitric Oxide, 2012. 26(4): p. 285-294.

24. Reidy, M.A., Factors controlling smooth-muscle cell proliferation. Arch Pathol Lab Med, 1992. 116(12): p. 1276-80.

25. Milleret, V., et al., Influence of the fiber diameter and surface roughness of electrospun vascular grafts on blood activation. Acta biomaterialia, 2012. 8(12): p. 4349-4356.

26. Chen, Y.M., et al., Cultivation of endothelial cells on adhesive protein-free synthetic polymer gels. Biomaterials, 2005. 26(22): p. 4588-4596.

27. Chen, Y.M., et al., Platelet adhesion to human umbilical vein endothelial cells cultured on anionic hydrogel scaffolds. Biomaterials, 2007. 28(10): p. 1752-1760.

28. Nojiri, C., et al., In vivo protein adsorption on polymers: visualization of adsorbed proteins on vascular implants in dogs. J Biomater Sci Polym Ed, 1992. 4(2): p. 75-88.

29. Hibino, N., et al., A critical role for macrophages in neovessel formation and the development of stenosis in tissue-engineered vascular grafts. The FASEB Journal, 2011. 25(12): p. 4253-4263.

30. Spiller, K.L., et al., The role of macrophage phenotype in vascularization of tissue engineering scaffolds. Biomaterials, (0). 


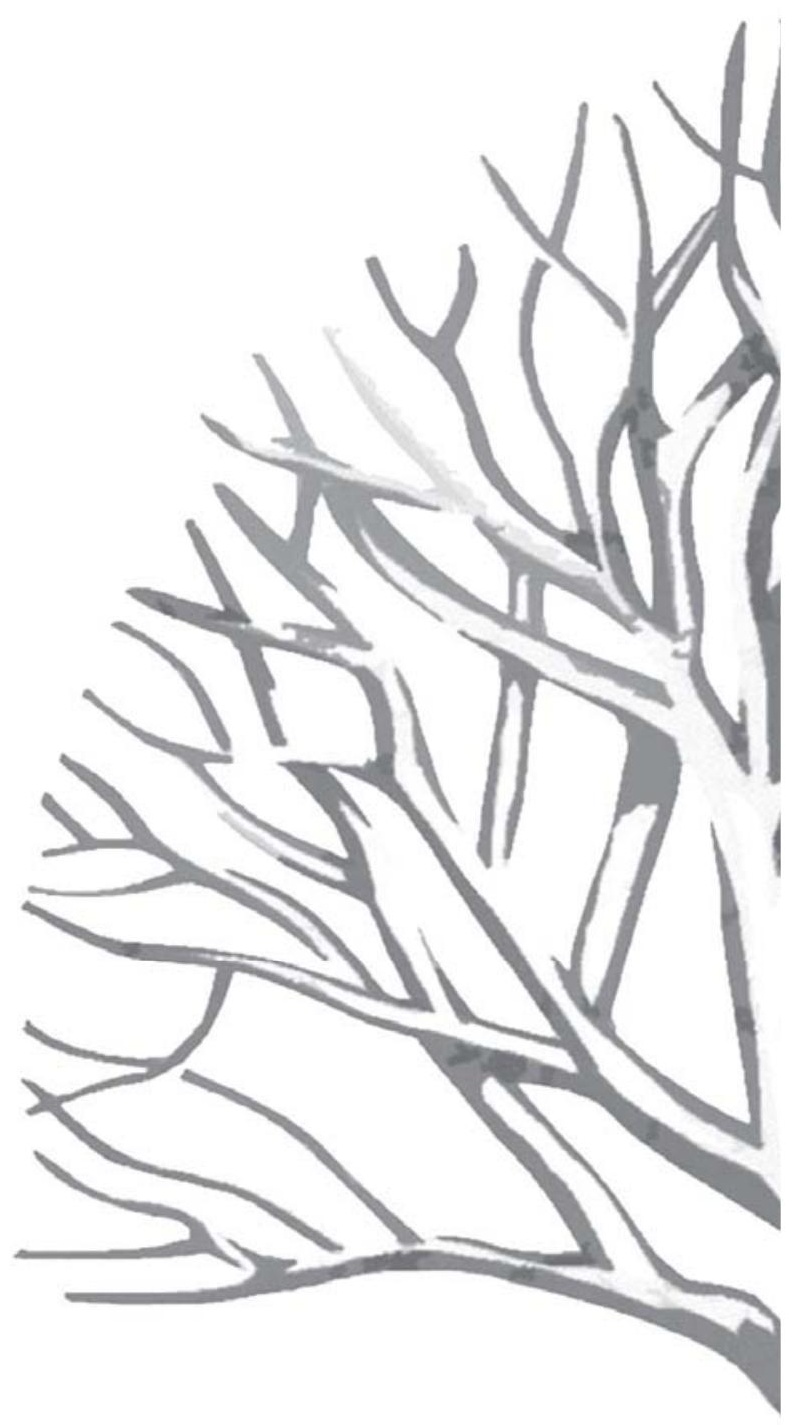




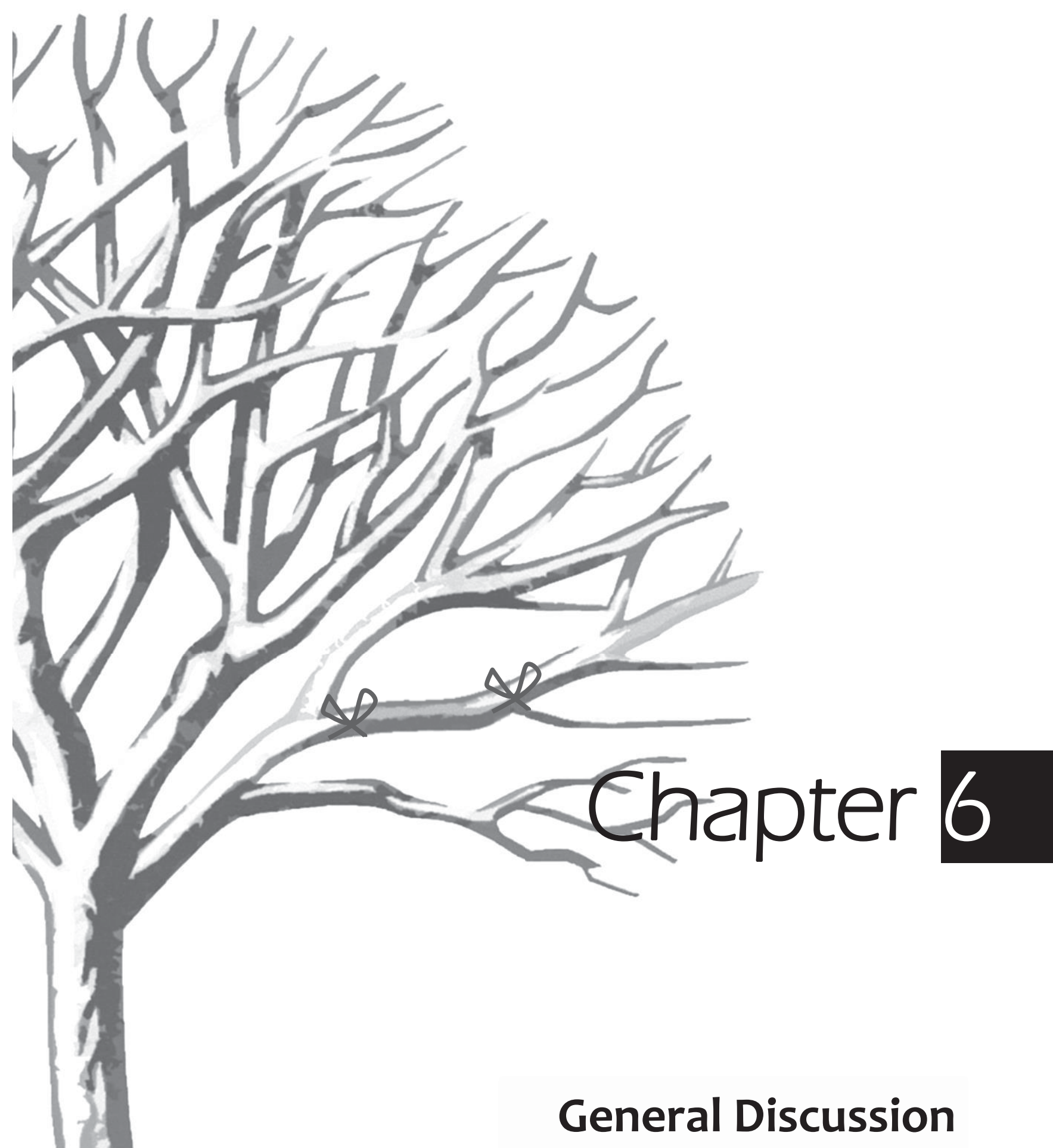




\section{Summary of main results}

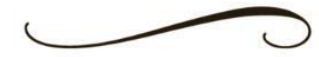

When an electro-responsive Polyacrylic acid.Fibrin (PAA/Fibrin) hydrogels is exposed to an electrical field, attraction of ions toward electrodes produces conformational deformation of the hydrogel. As a result, cell penetration is enhanced likely due to micro-scale swelling/deswelling of the hydrogel. Additionally, continued swelling/deswelling of the hydrogel produces mechanical force which reorganizes the embedded Fibrin into aligned bundles. Reorganization of Fibrin produces a micro-patterned bioactive surface for cells and navigates their alignment. The aligned cytoskeleton of cells will induce phenotypic responses such as smooth muscle cells differentiating into a contractile phenotype. However, other regulatory factors such as the actual produced mechanical force may still play a role in these cellular phenotype changes. Increased collagen production in the stimulated hydrogels is a reflection of a synthetic phenotype change of the cells. Therefore the populated hydrogel ends up with a phenotypic mix of smooth muscle cells (Fig. 1). Both phenotypes are useful for TE constructs. Upon in vivo implantation, electro-responsive PAA/Fibrin coating of electro-spun PCL vascular graft provides a relevant context that could improve arterial graft remodeling by promoting endothelialization and repopulation of cells. 


\section{Cell distribution in TE constructs}

Heterogeneous distribution of cells in the scaffolds is one of the most common and major limitations in providing homogenous engineered tissue. Uniform cell distribution in the tissue engineered (TE) constructs is therefore one of the important measures to accomplish uniform tissue growth in the structure [1, 2]. It has been shown that biophysical and biochemical interactions between cells and the surrounding extracellular environment can strongly modulate cell motility in three dimensional scaffolds [3-5]. Our proposed culturing system is based on an electro-responsive hydrogel, which induces infiltration and homogeneity of cells in the structure by indirect low voltage electrical field $\left(0.06 \mathrm{v} / \mathrm{mm}, 1 \mathrm{mV} / \mathrm{cm}^{2}, 0.0167 \mathrm{~Hz}\right)$ (chapter 2$)$. In our studies, the homogenous distribution of cells remained consistent up to $4 \mathrm{wks}$ of in vitro culture (chapter 4). We investigated the direct role of applied electrical field on cell distribution by blocking voltage gated L-type $\mathrm{Ca}^{2+}$-channel of SMCs with Verapamil. Verapamil had a negligible effect on the distribution of cells over the depth of the structure, so we concluded that direct activation of SMCs by electrical stimulation is not important for their motility, but rather that the continuous deformation of the PAA/Fibrin hydrogel is likely the major cause of SMCS distribution after seeding. This, in effect, could be similar to the micro-scale vacuum system used to facilitate the mobility of cells in vitro [6, 7]. In addition, when a polyelectrolyte hydrogel like PAA/Fibrin is exposed to an electrical field, a gradient of swelling ratio and local $\mathrm{pH}$ is generated in the structure which in turn could influence cell behavior [8, 9]. Supporting this interpretation, L.Han et al. recently showed directed migration of SMCs towards lower hydrated area when seeded on a swelling gradient polyelectrolyte multilayer [10]. In addition, migration of mobile ions and dissociation of carboxylic groups in PAA/Fibrin hydrogel during electrical stimulation can create an ion enrichment/depletion region within the hydrogel, which subsequently can alter local $\mathrm{pH}[11,12]$. A pH gradient in the intracellular and extracellular environment of cell-substrate interface has been shown to facilitate cell migration [13, 14]. For instance, acidic extracellular $\mathrm{pH}$ have been reported to promote migration of cells by regulating activation of $\alpha_{v} \beta_{3}$ integrin [15]. We suggest that higher distribution of cells in electrically stimulated PAA/Fibrin hydrogel is not only driven by semi-pumping swelling/deswelling behavior of the hydrogel, but is also achieved by induced chemical and physical gradients in the hydrogel, 
which facilitate migration of cells into the structure. Our in vivo findings on higher cell density in electrically stimulated acellular PAA/Fibrin coated PCL implanted grafts also support the effect of micro-scaled changes in the structure to promote cell migration (chapter 5), while physical deformation of $\mathrm{PAA} /$ Fibrin hydrogel in an electrical field is negligible in view of the mechanical properties of $\mathrm{PCL}$ but also in comparison with the already appreciable hemodynamic mechanical conditioning in vivo. However, further experiments are needed to elucidate these mechanisms.

Our proposed system based on electro-responsive hydrogel can also be considered an efficient way for in vitro 3D cell seeding compared to static and dynamic seeding methods which are indeed highly dependent on the interconnectivity and porosity of the scaffold [16-18], and might result in destruction of cells and scaffold thereby affecting cell density and morphology $[19,20]$.

\section{Architectural patterning of engineered tissue}

Alignment and organization of cells in their extracellular matrix are essential factors for good functionality of tissue-engineered blood vessels. In a natural blood vessel, endothelial cells are aligned along the vessel length while smooth muscle cells align in a circumferential multilayer pattern. The appropriate anatomical orientation of cells controls the vessel response to blood flow and pressure. External signals of mechanical stretch [21], shear flow [22] and electrical field [23] as well as pre-patterning of seeding surface and substrate [24] are major inducers of cell and fiber alignment in vitro. In our study, electrical stimulation of PAA/Fibrin hydrogels showed high alignment in orientation of seeded SMCS nucleus and actin filaments (chapter 2). In addition, PAA/Fibrin hydrogel showed changes in microtopography of Fibrin by reorganization of randomly dispersed Fibrin fibers into aligned bundle-like structures after electrical stimulation (chapter 2). Thus, orientation of cells could be induced either by applied electrical field, produced mechanical strain during responsive swelling/deswelling or induced microtopography of Fibrin fibers. We suggest cell alignment was not regulated by a direct effect of the electrical field on contraction of cells as blocking voltage gated calcium channels had no significant effect on SMCs/actin filament orientation (chapter 2). Examination of cells seeded and stimulated on PAA only hydrogel revealed no major preference in direction of SMCs/actin filament despite the mechanical force produced by hydrogel during electrical stimulation (chapter 3 ). This data 
suggest that SMCs and actin filaments require microtopography of Fibrin fibers for alignment and not the mechanical strain produced by hydrogel. The dominance of topography over mechanical forces in directing cell alignment has been reported also by others. For instance Houtches et al. investigated the combined effect of topography and cyclic strain on orientation of smooth muscle cells where cells showed to follow oriented microgrooves in the structure rather than the applied strain [25]. Likewise, a similar study in fibroblasts by Ahmed et al. indicated that the orientation of cell nuclei followed the direction of patterned lines in presence of different tensile strain axis [26]. Fibrin provides cell-binding sites for the cells in the PAA/Fibrin substrate and therefore could reorganize the cytoskeleton in response to induced geometrical changes. The tension induced by actin fibers on focal adhesion is proposed to be the key element of cell response to microenvironment changes [27]. However, pre-aligned Fibrin fibers in pre-stimulated PAA/Fibrin hydrogel were not able to induce significant alignment in SMCs/actin filaments (unpublished data). This data suggests Fibrin fibers could induce alignment in cells and actin filaments if only reorganized after seeding. A study by Matsumoto et al. on alignment of myoblasts and endothelial cells in continuously stretched Fibrin gels, further supports the importance of Fibrin fibrils orientation structure in inducing contact guidance cues leading to cell alignment [28]. We observed similar effects in Fibrin organization and SMCs/actin filaments alignment along the strain direction when PAA/Fibrin hydrogels were mechanically stretched by the Flexcell system. Based on these data, we propose reorganization of Fibrin structure in electrically stimulated PAA/Fibrin is induced by mechanical force of swelling/deswelling of electrically stimulated PAA/Fibrin (chapter 3). Controlling the Fibrin structure and subsequent alignment of cells through contact guidance by mechanical stretch however will be limited by need of other external forces or limited space for the structure to move and strain. In vivo, electrical stimulation of implanted PAA/Fibrin coated PCL grafts in our study prevented Fibrin alignment to guide cell orientation likely due to the presence of a strong backbone structure of electro-spun PCL. Therefore, fabricating aligned electro-spun grafts with microfibers and microgrooves could promote cell and ECM organizations [29, 30].

In addition, tissue architecture could also be achieved by self-reorganization of produced extracellular matrix through activity of enzymes such as proteases expressed by cells [31]. Matrix metalloproteinases (MMPs) are a big family of enzymes responsible for degrading and remodeling of tissues [32]. 


\section{- Role of matrix metalloproteinase activity}

Regulation of MMPs can profoundly affect cell behavior by remodeling their surrounding environment through degradation and organization of the present and newly synthesized ECM [33-35]. MMP-2 and MMP-9 are the two major gelatinases of the MMP family that are expressed by vascular smooth muscle cells [36]. Mechanical strain has been reported to enhance MMP-2 activity and facilitate remodeling of the vascular substrate [37-39]. We also observed higher activity of MMP-2 after electrically stimulating the hydrogels. The higher expression of MMP-2 in electrically stimulated hydrogels with reorganized collagen with network structure while non-stimulated samples with lower MMP-2 activity revealed local spot deposition of collagen (Chapter 3). We hypothesize that increased collagen remodeling by MMP-2, together with increased collagen production, has led to optimized organization and improvement in mechanical properties of stimulated PAA/Fibrin samples. We ruled out electrical stimulation itself as a modulator of MMP-2 activity as electrically stimulated Fibrin gels showed no significant difference in MMP-2 activity compared to controls (chapter 3). Interestingly, applying mechanical force (comparable with produced mechanical stretch by PAA hydrogel in response to electrical field) to Fibrin gels in Flexcell system did not induce MMP-2 activity either (unpublished data). Similar observation has been recently reported by Won.se et al. where MMP-2 expression of vascular smooth muscle cell was not affected by 2 hrs exposure of mechanical stretching of Flexcell system, but significantly increased after 3 hours of stimulation [40]. These data suggests certain level of mechanical stimulation is needed to activate the signaling pathways for MMP-2 production and 2 hrs of stimulation might not be long enough [40]. Therefore other regulatory process might also be involved in this process.

Our result on higher penetration of cells into the structure in electrically stimulated samples compared to control ones was also consistent with higher activity of MMP-2 (chapter 2).

MMP-2 can also enhance the migration of cells into vascular tissue by degrading basal membrane proteins [41]. A study by Ahmed et al. demonstrated high activity of Plasmin during Fibrin gel breakdown [42]. Plasmin has been shown to activate MMP-2 activity [43, 44]. Thus, we speculate that MMP-2 upregulation could also be mediated by Plasmin expression released during proteolytic breakdown of Fibrin. Formal proof of 
Plasmin and MMP-2 involvement in these processes would require selective inhibition of their activities. Furthermore, verapamil intervention showed that the effect was modulated via a calcium independent pathway (chapter 3).

\section{Phenotype regulation of Smooth muscle cells}

In vivo, smooth muscle cells in the media of a blood vessel show a quiescent, contractile phenotype [45]. However, they have the ability to switch their phenotype into synthetic in response to environmental stimulus [46]. We investigated SMC phenotype in our culture systems in response to electrical and mechanical stimulation (chapter 4). Our results are based on Western blot analysis of contractile proteins including smooth muscle alpha-actin, smooth muscle cell myosin heavy chain (SMMHC) and Calponin in SMCs seeded PAA/Fibrin hydrogels and Fibrin gels. Electrical stimulation increased contractility of SMCs phenotype seeded on PAA/Fibrin hydrogels by significant enhancement of the expression of contractile proteins (alpha-actin, SMMHC and Calponin) after 1 week of culture. Blocking voltage gated calcium channels during electrical stimulation of PAA/Fibrin hydrogel suggested SMMHC upregulation was mediated via Calcium dependent pathway whereas alphaactin and Calponin expression were calcium independent.

Patterning of SMCs induced by an aligned structure has been reportedly shown to induce a contractile phenotype [47-49]. We suggest contact guidance of SMCs and Fibrin fibers to regulate contractile gene expression in the cell [50, 51].

Additionally, SMCs in PAA/Fibrin hydrogels exposed to electrical stimulation showed significantly higher collagen production in the bulk of the samples compared to non-stimulated control ones after 4 weeks of culture (1stimulation/week)(chapter 4). This behavior, however, suggest a more synthetic phenotype of smooth muscle cells. Evaluating gene expression in SMCs seeded in PAA/Fibrin demonstrated upregulation both in contractile markers as well as collagen genes after 1 and 3 weeks of culture (unpublished data). This apparent contradiction in the phenotypic state of SMCs has been reported also by others $[52,53]$. To achieve functional vascular grafts, however, both ECM deposition and contractile behavior of SMCs indicated to synthetic and contractile phenotype are crucial factors [54]. 


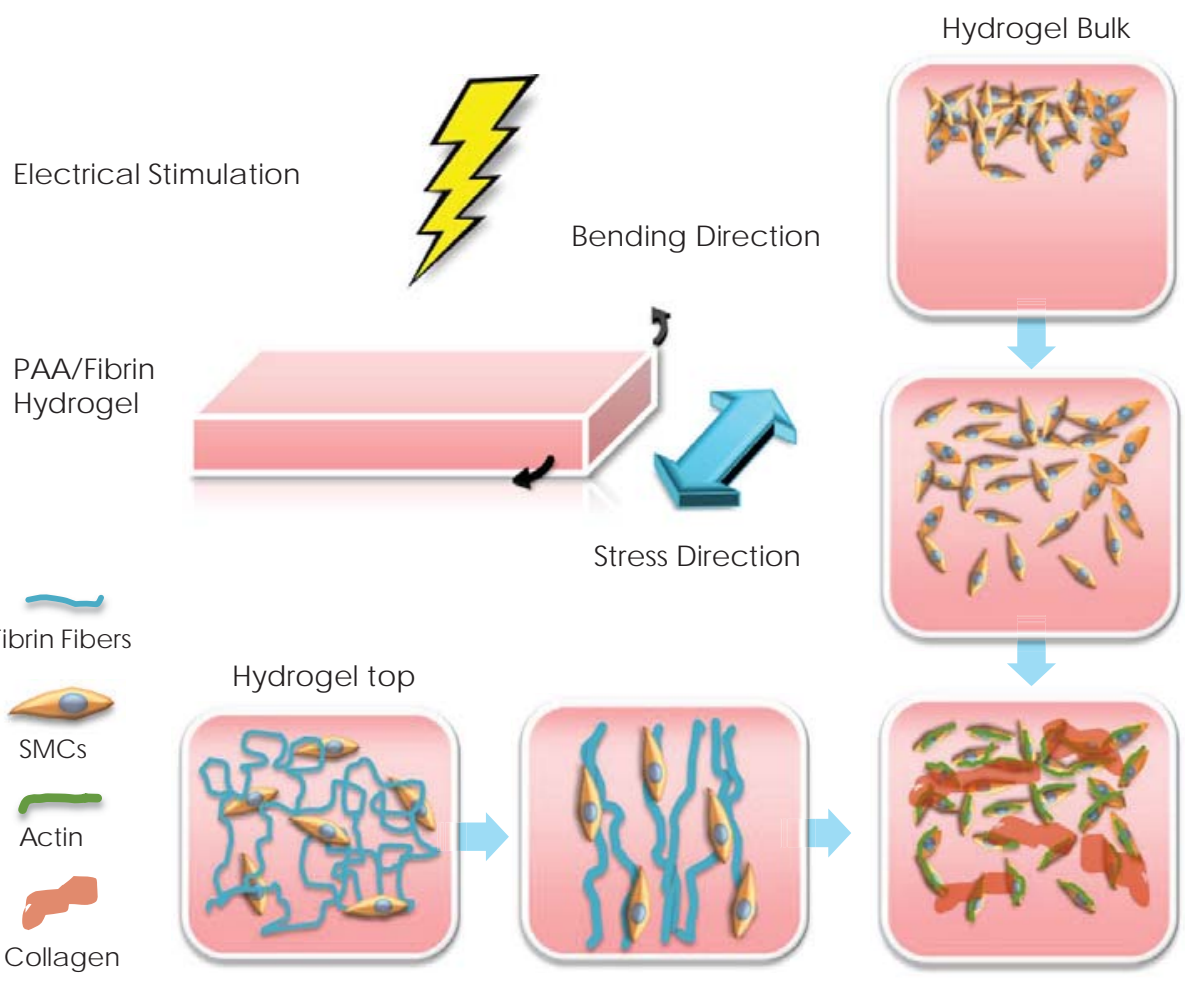

Fig 1. Schematic of SMCs seeded PAA/Fibrin hydrogel exposed to electrical stimulation. Induced physical and chemical changes of the hydrogel promote infiltration and homogeneity of cells into the bulk of the hydrogel. Induced mechanical force within the hydrogel aligned Fibrin fibers and subsequently cells and their alpha-actin filaments.

\section{Implantation of vascular graft}

Our proposed system has the potential to be translated into in vivo clinical applications. In developing a tissue engineered vascular graft, guiding cell performance and tissue formation by the recipient is the major goal when implanting acellular scaffolds. Yet, in vivo results have revealed insufficient cell migration and poor tissue formation within current available scaffolds [55]. One possible approach to improve the implanted graft is the implantation of a pre-seeded scaffold in the body [56]. However, autologous cell harvesting, lengthy cell expansion and limited cell sources in patients with a history of 
disease, pose limitations on clinical application of autologous seeded scaffolds [56-58]. For widespread acceptance of tissue engineered vascular grafts they need to be easy to make, cheap and preferably available off-the-shelf [59]. If the latter is not feasible, production time should be short so that patients in critical condition still benefit from them.

Applying external signals could play an important role in guiding cell behavior and accelerating graft preparation [60]. To our knowledge there is no published report on application of external signals to enhance cell infiltration in an already implanted acellular vascular graft. However, several studies have been conducted on growing a vascular scaffold using the peritoneum cavity as bioreactor [61-64]. The formed tissue is produced by a foreign body reaction where the hematopoietic cells differentiated gradually to build layers of myofibroblasts, collagen matrix and mesothelial cells coverage [65]. Stickler et al. investigated the effect of external mechanical forces to improve the development and growth of the vascular graft in the peritoneum [66]. Cells from the peritoneal cavity were attracted to a tubular scaffold by a designed cyclic stretching device connected to a pulsatile pump. Pulsed stretching significantly increased collagen organization, F-actin expression and mechanical properties of the scaffolds [66]. Although an interesting approach, intraperitoneal implantation of a pulsed devise and the long time needed to create such a graft gives limitations for clinical usage.

We developed our implantation system based on PAA/Fibrin hydrogel to control cell behavior into higher integration with the graft and surrounding vascular tissue by external signals. During in vivo application, neighboring cells are triggered to migrate into the acellular graft and produce extracellular matrix while inhibiting neointima formation. The PAA/Fibrin hydrogel structure is by itself not strong enough to withstand physiological mechanical forces of blood flow. Therefore, hydrogel was used as coating to functionalize electrospun PCL grafts to obtain an electro-responsive scaffold with sufficient mechanical strength for in vivo application. PCL grafts have been reported to show promising results as substitutes of small diameter vessels. They provide a mechanically stable structure with good retention of sutures suitable for implantation in vascular system. Although PAA coating of PCL is an attractive strategy with promising results, the use of combinations of polymers also present challenges from a biocompatibility and regulatory point of view $[67$, 68]. 


\section{- Vascular graft-body interactions}

Surface charge, energy and roughness are determining characteristics of the luminal face of the graft, which affect interaction between blood cells and graft material. As red and white blood cells and platelets have negatively charged surfaces, [69] a negative graft surface charge attenuates platelet adhesion and potentially minimizes the contact between red and white blood cells and the graft [70]. Thrombosis is one of the major causes of small blood vessel failure in vivo [71]. Coating vascular grafts with negatively charged materials has been used to reduce surface thrombogenicity since 1960 [72]. Polyelectrolytes are known materials to exhibit negative charge surface thus having a potential as surface modification to reduce thrombogenicity in bloodcontact biomaterials [73]. Polyacrylic acid coating has been shown to reduce platelet adhesion due to its highly negative charges and hydration degree [74]. Additionally, negative charge surface could enhance adhesion of serum proteins into the substrate. Kikuchi et.al reported strong correlation of serum Fibronectin and Vitronectin adsorption with Endothelial cell spreading and growth profile on polyamine copolymer surfaces [75]. We also found higher coverage of endothelial cells in electrically stimulated PCL grafts coated with anionic PAA/Fibrin hydrogel compared to non-stimulated samples after 4 weeks of implantation (chapter 5).

When a PCL graft with charged functional groups of - $\mathrm{COOH}$ is exposed to an electrical field a series of events occurs. Carboxylic group dissociate, and cations migrate to the anode side, and the repelling forces between negatively fixed charges induce changes in ionic concentration at the graft and bloodstream boundaries. Consequently, the electrostatic properties of surface will change. Surface zeta potential could determine the electrostatic interactions between bioactive compounds and the polymer surface [76, 77]. We suggest increasing negative charge density and mobility of carboxylic groups when PAA/Fibrin/PCL graft is stimulated by electrical field could enhance adhesion and growth of Endothelical cells by forming layer of adsorbed protein in the surface. Our hypothesis is supported by studies of Zue.et al and Wachem et.al which show enhanced Endothelial cell adhesion to negatively charged surface through adsorbed protein layer [78, 79]. Furthermore, negatively charged carboxylic acid could also bind to positively charged surface regions of growth factors such as VEGF coated surfaces [80]. Understanding protein adsorption to charged surfaces is important for tissue engineering as selective cell-adhesive proteins can affect cell behavior and 
function in the scaffold [81-83]. Adsorbed proteins could also be released when the hydrogel is externally stimulated by an electrical field. In fact, the ionizable carboxylic group of acrylic acid can demonstrate some degree of dissociation and association regarding the conditions in their environment [84]. Recently, Delcroix et al. presented a switchable protein absorbent surface by changing $\mathrm{pH}$, using mixed brushes of Polyethylene Oxide and PAA [85].

In addition, negative surfaces could initiate the intrinsic coagulation pathway through contact activation and lead to thrombosis and Fibrin formation [86, 87]. Although the clinical importance of the intrinsic coagulation pathway has been challenged, our research group recently showed that intrinsic activation occurs on patient-derived endothelial cell cultures [88]. Fibrin, in cooperation with the adsorbed protein, provide a suitable matrix for cells as the first steps of neointima formation [89]. Our observations where PAA/Fibrin coated PCL grafts have a higher degree of neointima formation compared to bare $\mathrm{PCL}$ grafts could be explained by such a mechanism. Electrical stimulation of PAA/Fibrin coated PCL grafts significantly prevented neointimal growth compared to non-stimulated coated grafts, perhaps due to shedding of adsorped proteins (chapter 5). However the exact role and mechanisms of these factors is still unknown and further studies are necessary (Fig.2).

In vivo, MMP-2 has been also shown to play an important role in expression of pro-angiogenic factors such as vascular endothelial growth factors (VEGF) and therefore regulate neovascularization [90]. Higher coverage of Endothelial cells as well as a higher density of myofibroblast type cells in the stimulated PAA/Fibrin/PCL grafts could thus also be explained by higher activity of MMP-2 induced as a result of the responsive to the electrical field. Studies with more comprehensive gene and protein expression arrays would resolve some of these mechanisms. Rosa et al. reported a significant effect of surface charge of cationic polyelectrolyte substrate on MMP activity in fibroblasts [91]. Further studies on the regulation of MMPs and anionic polyelectrolytes could shed more light on this interaction, which might be beneficial in controlling cell migration in tissue engineered constructs. 


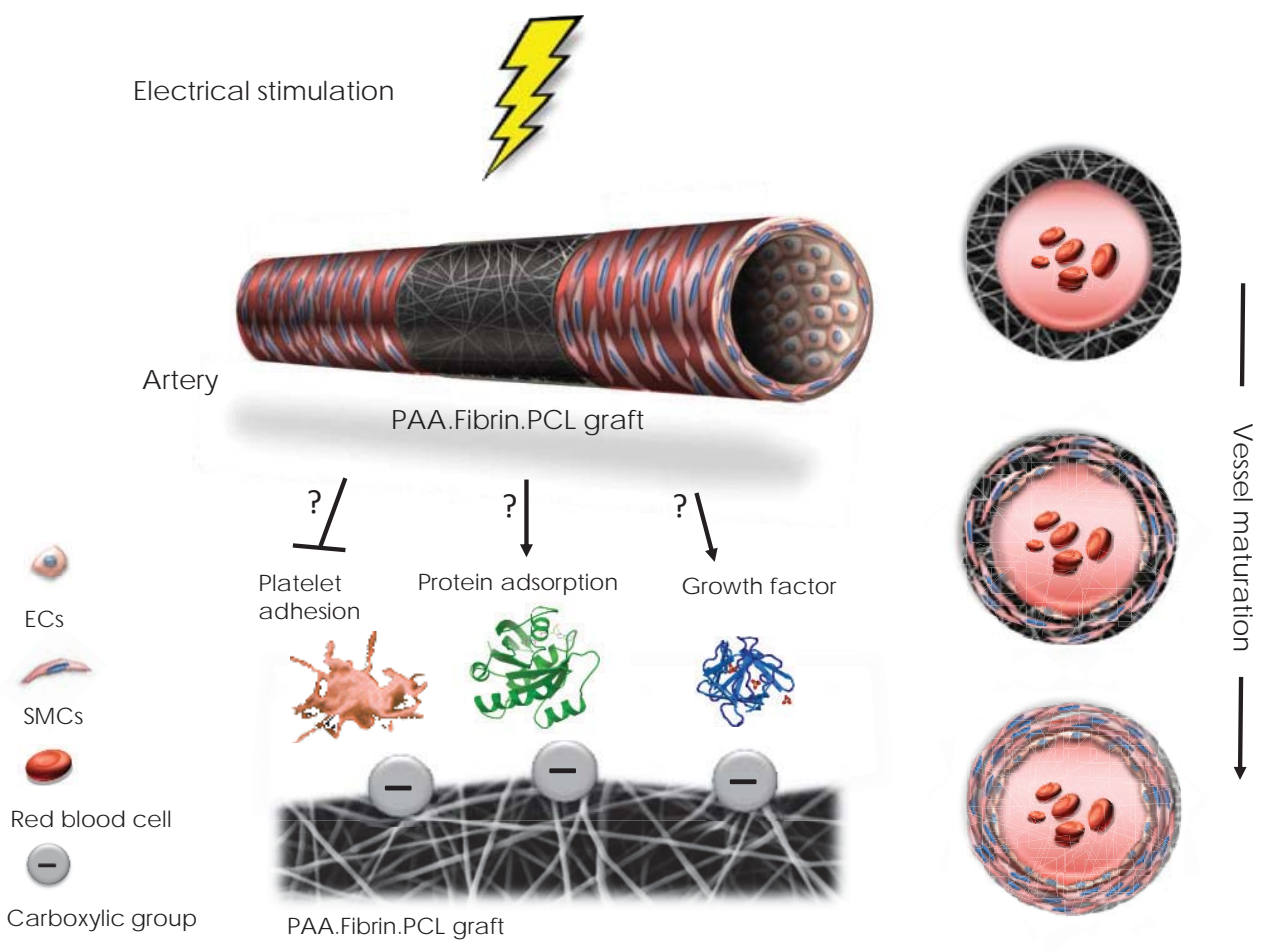

Fig.2. Schematic of implanted electro-responsive PAA.Fibrin coated PCL graft exposed to electrical stimulation. Conformational changes of the hydrogel structure could regulate vessel maturation through possible interaction of the provided functional surface and its physiological environment (e.g. circulating platelets, proteins and growth factors)

\section{Conclusion and future work}

The presented work describes our investigations of external stimulation of polyanionic materials as substrate for tissue engineering applications. The developed system enabled profound control of cell behavior towards more functional tissue. Although more research is needed to better define the regulation of ion transports, flow dynamics and surface charges induced by electrical stimulation, initial mechanistic studies presented here suggest that fast reorganization of the hydrogel with its embedded Fibrin fibers drive cell alignment. These findings could be highly beneficial for the development of an easily tunable vascular tissue graft. 
In vitro, we showed the effect of stimulation only on smooth muscle cells, while in vivo both endothelial and smooth muscle cells simultaneously populate a graft or hydrogel and are important to be studied [92]. In order to fully understand how ECs and SMCs are regulating each other in our electroresponsive system, in vitro studies with co-cultures of ECs and SMCs are highly recommended. Electrically stimulation of PAA/Fibrin hydrogels showed profound deposition of collagen as well as enhanced mechanical properties (i.e. tensile strength). Evaluating collagen production and mechanical properties of implanted Fibrin/PAA/PCL grafts will help us gain better insight in the ability of cells to produce ECM and regenerate vessel tissue. As elastin is a crucial protein in ECM and essential for tissue function, vascular elasticity also needs to be determined in our tissue engineered constructs in vitro and in vivo [93].

Polyacrylic acid, Fibrin and PCL used as constructive materials for fabrication of tissue engineered structures in this study have been reported as biocompatible biomaterials used widely for biomedical application [94-96]. Indeed, we did not observe any toxicity or acute inflammation in in vitro and in vivo experiments. However, in addition to biocompatibility, biomaterials need to be biodegradable for clinical applications, so that remodeling of tissue depends on the balance between degradation and formation of structural proteins by infiltrated cells. Our developed polyacrylic acid hydrogel was based on covalent binding of the acrylic acid monomer by MBAA as the cross-linker, which results in a non-degradable structure and therefore limit the clinical application of these hydrogels. Currently we are working on electro-sensitive polyphosphoester (PPE) based hydrogels produced within the Interreg EMR BioMIMedics project. These PPE hydrogels are biodegradable and carry negative charges, which make them suitable candidates for electro-responsive applications.

Next to regulating cell function by induced deformation of electro-responsive hydrogels these materials could also be used for drug delivery [97]. Polyelectrolytes as smart carriers for biomolecules and drugs are strong tools to control cell-matrix interactions and even further tissue generations. Imbedded components such as growth factors or other proteins and applying prolonged and multiple component release could improve the regeneration process by activating desired pathways in the developing tissue. Indeed, electro-responsive hydrogels could serve as multi-functional, intelligent substrates in a wide variety of tissue engineering applications. 


\section{References}

1. Wendt, D., et al., Uniform tissues engineered by seeding and culturing cells in 3D scaffolds under perfusion at defined oxygen tensions. Biorheology, 2006. 43(3-4): p. 481-8.

2. Wendt, D., et al., Oscillating perfusion of cell suspensions through three-dimensional scaffolds enhances cell seeding efficiency and uniformity. Biotechnology and Bioengineering, 2003. 84(2): p. 205-214.

3. Harley, B.A., et al., Microarchitecture of three-dimensional scaffolds influences cell migration behavior via junction interactions. Biophysical Journal, 2008. 95(8): p. 401324.

4. Peyton, S.R., et al., Marrow-Derived stem cell motility in 3D synthetic scaffold is governed by geometry along with adhesivity and stiffness. Biotechnology and Bioengineering, 2011. 108(5): p. 1181-1193.

5. Zaman, M.H., et al., Migration of tumor cells in 3D matrices is governed by matrix stiffness along with cell-matrix adhesion and proteolysis. Proceedings of the National Academy of Sciences, 2006.103(29): p. 10889-10894.

6. Nieponice, A., et al., Development of a tissue-engineered vascular graft combining a biodegradable scaffold, muscle-derived stem cells and a rotational vacuum seeding technique. Biomaterials, 2008. 29(7): p. 825-33.

7. Chen, M., H. Michaud, and S. Bhowmick, Controlled vacuum seeding as a means of generating uniform cellular distribution in electrospun polycaprolactone ( $P C L)$ scaffolds. Journal of biomechanical engineering, 2009. 131(7): p. 074521.

8. Yao, K. and S. Sun, Bending behavior of an electrically activated complex forming hydrogel. Polymer Bulletin, 1992. 28(6): p. 677-681.

9. Lühmann, T. and H. Hall, Cell Guidance by 3D-Gradients in Hydrogel Matrices: Importance for Biomedical Applications. Materials, 2009. 2(3): p. 1058-1083.

10. Han, L., et al., Directional cell migration through cell-cell interaction on polyelectrolyte multilayers with swelling gradients. Biomaterials, 2013. 34(4): p. 975-84.

11. Glazer, P.J., et al., Role of $\mathrm{pH}$ gradients in the actuation of electro-responsive polyelectrolyte gels. Soft Matter, 2012. 8(16): p. 4421-4426.

12. O'Grady, M.L., P.L. Kuo, and K.K. Parker, Optimization of electroactive hydrogel actuators. ACS Appl Mater Interfaces, 2010. 2(2): p. 343-6.

13. Martin, C., et al., Intracellular pH gradients in migrating cells. Am J Physiol Cell Physiol, 2011. 300(3): p. C490-5.

14. Paradise, R.K., et al., Directional cell migration in an extracellular $\mathrm{pH}$ gradient: a model study with an engineered cell line and primary microvascular endothelial cells. Exp Cell Res, 2013. 319(4): p. 487-97.

15. Paradise, R.K., D.A. Lauffenburger, and K.J. Van Vliet, Acidic extracellular $p H$ promotes activation of integrin alpha(v)beta(3). PLoS ONE, 2011. 6(1): p. e15746.

16. Thevenot, P., et al., Method to analyze three-dimensional cell distribution and infiltration in degradable scaffolds. Tissue engineering. Part C, Methods, 2008. 14(4): p. 319-31.

17. Martin, I., D. Wendt, and M. Heberer, The role of bioreactors in tissue engineering. Trends in biotechnology, 2004. 22(2): p. 80-6.

18. Roh, J.D., et al., Centrifugal seeding increases seeding efficiency and cellular distribution of bone marrow stromal cells in porous biodegradable scaffolds. Tissue engineering, 2007. 13(11): p. 2743-9. 
19. Villalona, G.A., et al., Cell-seeding techniques in vascular tissue engineering. Tissue engineering. Part B, Reviews, 2010. 16(3): p. 341-50.

20. Kim, B.S., et al., Optimizing seeding and culture methods to engineer smooth muscle tissue on biodegradable polymer matrices. Biotechnology and Bioengineering, 1998. 57(1): p. 46-54.

21. Kuzuya, M., et al., Deficiency of Gelatinase A Suppresses Smooth Muscle Cell Invasion and Development of Experimental Intimal Hyperplasia. Circulation, 2003. 108(11): p. 1375-1381.

22. Lee, A.A., et al., Fluid shear stress-induced alignment of cultured vascular smooth muscle cells. Journal of biomechanical engineering, 2002. 124(1): p. 37-43.

23. Tandon, N., et al., Alignment and elongation of human adipose-derived stem cells in response to direct-current electrical stimulation. Conference proceedings : ... Annual International Conference of the IEEE Engineering in Medicine and Biology Society. IEEE Engineering in Medicine and Biology Society. Conference, 2009. 2009: p. 651721.

24. Williams, C., et al., The use of micropatterning to control smooth muscle myosin heavy chain expression and limit the response to transforming growth factor 81 in vascular smooth muscle cells. Biomaterials, 2011. 32(2): p. 410-418.

25. Houtchens, G.R., et al., Combined effects of microtopography and cyclic strain on vascular smooth muscle cell orientation. Journal of biomechanics, 2008. 41(4): p. 7629.

26. Ahmed, W.W., et al., Myoblast morphology and organization on biochemically micropatterned hydrogel coatings under cyclic mechanical strain. Biomaterials, 2010. 31(2): p. 250-8.

27. Greiner, A.M., et al., Cyclic tensile strain controls cell shape and directs actin stress fiber formation and focal adhesion alignment in spreading cells. PLoS ONE, 2013. 8(10): p. e77328.

28. Matsumoto, T., et al., Three-dimensional cell and tissue patterning in a strained fibrin gel system. PLoS ONE, 2007. 2(11): p. e1211.

29. Whited, B.M. and M.N. Rylander, The influence of electrospun scaffold topography on endothelial cell morphology, alignment, and adhesion in response to fluid flow. Biotechnology and Bioengineering, 2014. 111(1): p. 184-195.

30. Uttayarat, P., et al., Micropatterning of three-dimensional electrospun polyurethane vascular grafts. Acta Biomater, 2010. 6(11): p. 4229-37.

31. Keely, P.J., Proteolytic remodeling of the ECM and the geometric control of stem cell fate. Dev Cell, 2013. 25(4): p. 325-6.

32. Johnson, J.L., et al., Matrix Metalloproteinase (MMP)-3 Activates MMP-9 Mediated Vascular Smooth Muscle Cell Migration and Neointima Formation in Mice. Arteriosclerosis, Thrombosis, and Vascular Biology, 2011. 31(9): p. e35-e44.

33. Rodríguez, D., C.J. Morrison, and C.M. Overall, Matrix metalloproteinases: What do they not do? New substrates and biological roles identified by murine models and proteomics. Biochimica et Biophysica Acta (BBA) - Molecular Cell Research, 2010. 1803(1): p. 39-54.

34. Galis, Z.S. and J.J. Khatri, Matrix metalloproteinases in vascular remodeling and atherogenesis: the good, the bad, and the ugly. Circ Res, 2002. 90(3): p. 251-62.

35. Dikovsky, D., H. Bianco-Peled, and D. Seliktar, Defining the Role of Matrix Compliance and Proteolysis in Three-Dimensional Cell Spreading and Remodeling. Biophysical Journal, 2008. 94(7): p. 2914-2925. 
36. Shimokawa, K.-i., et al., Matrix metalloproteinase (MMP)-2 and MMP-9 activities in human seminal plasma. Molecular Human Reproduction, 2002. 8(1): p. 32-36.

37. Seliktar, D., R.M. Nerem, and Z.S. Galis, Mechanical strain-stimulated remodeling of tissue-engineered blood vessel constructs. Tissue engineering, 2003. 9(4): p. 657-66.

38. Asanuma, K., et al., Uniaxial strain upregulates matrix-degrading enzymes produced by human vascular smooth muscle cells. Am J Physiol Heart Circ Physiol, 2003. 284(5): p. $\mathrm{H} 1778-84$.

39. Grote, K., et al., Mechanical stretch enhances MRNA expression and proenzyme release of matrix metalloproteinase-2 (MMP-2) via $N A D(P) H$ oxidase-derived reactive oxygen species. Circulation research, 2003. 92(11): p. e80-6.

40. Seo, K.W., et al., Mechanical stretch increases MMP-2 production in vascular smooth muscle cells via activation of PDGFR-beta/Akt signaling pathway. PLoS ONE, 2013. 8(8): p. e70437.

41. Klein, T. and R. Bischoff, Physiology and pathophysiology of matrix metalloproteases. Amino acids, 2011. 41(2): p. 271-90.

42. Ahmed, T.A., M. Griffith, and M. Hincke, Characterization and inhibition of fibrin hydrogel-degrading enzymes during development of tissue engineering scaffolds. Tissue Eng, 2007.13(7): p. 1469-77.

43. Monea, S., et al., Plasmin activates pro-matrix metalloproteinase-2 with a membranetype 1 matrix metalloproteinase-dependent mechanism. J Cell Physiol, 2002. 192(2): p. 160-70.

44. Mazzieri, R., et al., Control of type IV collagenase activity by components of the urokinase-plasmin system: a regulatory mechanism with cell-bound reactants. EMBO J, 1997. 16(9): p. 2319-32.

45. Sho Shinohara, S.S., Takanori Kihara and Jun Miyake, Chapter 14: Regulation of Differentiated Phenotypes of Vascular Smooth Muscle Cells, in Current Basic and Pathological Approaches to the Function of Muscle Cells and Tissues - From Molecules to Humans, H. Sugi, Editor. 2012.

46. Stegemann, J.P., H. Hong, and R.M. Nerem, Mechanical, biochemical, and extracellular matrix effects on vascular smooth muscle cell phenotype. J Appl Physiol (1985), 2005. 98(6): p. 2321-7.

47. Cao, Y., et al., Regulating orientation and phenotype of primary vascular smooth muscle cells by biodegradable films patterned with arrays of microchannels and discontinuous microwalls. Biomaterials, 2010. 31(24): p. 6228-6238.

48. Alford, P.W., et al., Vascular smooth muscle contractility depends on cell shape. Integr Biol (Camb), 2011. 3(11): p. 1063-70.

49. Feinberg, A.W., et al., Controlling the contractile strength of engineered cardiac muscle by hierarchal tissue architecture. Biomaterials, 2012. 33(23): p. 5732-5741.

50. Rensen, S.S., P.A. Doevendans, and G.J. van Eys, Regulation and characteristics of vascular smooth muscle cell phenotypic diversity. Neth Heart J, 2007. 15(3): p. 100-8.

51. Liu, W.F., Mechanical regulation of cellular phenotype: implications for vascular tissue regeneration. Cardiovasc Res, 2012. 95(2): p. 215-22.

52. Birukov, K.G., et al., Stretch affects phenotype and proliferation of vascular smooth muscle cells. Mol Cell Biochem, 1995.144(2): p. 131-9.

53. Chang, S., et al., Phenotypic modulation of primary vascular smooth muscle cells by short-term culture on micropatterned substrate. PLoS One, 2014. 9(2): p. e88089. 
54. Stock, U.A., et al., Dynamics of extracellular matrix production and turnover in tissue engineered cardiovascular structures. Journal of Cellular Biochemistry, 2001. 81(2): p. 220-228.

55. Nieponice, A., et al., In vivo assessment of a tissue-engineered vascular graft combining a biodegradable elastomeric scaffold and muscle-derived stem cells in a rat model. Tissue engineering. Part A, 2010. 16(4): p. 1215-23.

56. Pawlowski, K.J., et al., Endothelial cell seeding of polymeric vascular grafts. Frontiers in bioscience : a journal and virtual library, 2004. 9: p. 1412-21.

57. Armour, A.D., et al., A comparison of human and porcine acellularized dermis: interactions with human fibroblasts in vitro. Plastic and reconstructive surgery, 2006. 117(3): p. 845-56.

58. Byrom, M.J., M.K. Ng, and P.G. Bannon, Biomechanics and biocompatibility of the perfect conduit-can we build one? Annals of cardiothoracic surgery, 2013. 2(4): p. 43543.

59. Sarkar, S., et al., Achieving the ideal properties for vascular bypass grafts using a tissue engineered approach: a review. Medical \& biological engineering \& computing, 2007. 45(4): p. 327-36.

60. Bilodeau, K. and D. Mantovani, Bioreactors for tissue engineering: focus on mechanical constraints. A comparative review. Tissue engineering, 2006. 12(8): p. 2367-83.

61. Bonvini, S., et al., The Peritoneum as a Natural Scaffold for Vascular Regeneration. PLOS ONE, 2012. 7(3): p. e33557.

62. Song, L., et al., Bioengineered vascular graft grown in the mouse peritoneal cavity. Journal of vascular surgery, 2010. 52(4): p. 994-1002, 1002 e1-2.

63. Campbell, J.H., J.L. Efendy, and G.R. Campbell, Novel Vascular Graft Grown Within Recipient's Own Peritoneal Cavity. Circulation research, 1999. 85(12): p. 1173-1178.

64. Cebotari, S., et al., Guided Tissue Regeneration of Vascular Grafts in the Peritoneal Cavity. Circulation research, 2002. 90(8): p. e71.

65. Chue, W.-L., et al., Dog peritoneal and pleural cavities as bioreactors to grow autologous vascular grafts. Journal of vascular surgery, 2004. 39(4): p. 859-867.

66. Stickler, P., et al., Cyclically stretching developing tissue in vivo enhances mechanical strength and organization of vascular grafts. Acta biomaterialia, 2010. 6(7): p. 244856.

67. Williams, D.F., On the mechanisms of biocompatibility. Biomaterials, 2008. 29(20): p. 2941-53.

68. Gunatillake, P.A. and R. Adhikari, Biodegradable synthetic polymers for tissue engineering. European cells \& materials, 2003. 5: p. 1-16; discussion 16.

69. Jan, K.M. and S. Chien, Role of surface electric charge in red blood cell interactions. The Journal of general physiology, 1973. 61(5): p. 638-54.

70. Sherwin Shang, L.w., Selection of materials for biomedical applications, in Handbook of Materials Selection, M. Kutz, Editor. 2002, John Wiley \& Sons.

71. Bos, G.W., et al., Small-diameter vascular graft prostheses: current status. Arch Physiol Biochem, 1998. 106(2): p. 100-15.

72. Part X: Cardivascular systems, in Principles of Tissue Engineering, R.L. Robert Lanza, Joseph P. Vacanti, Editor. 2011.

73. Ito, H., et al., In vivo and in vitro blood compatibility of polyelectrolyte complexes formed between cellulose derivatives. Journal of Applied Polymer Science, 1986. 32(2): p. 3413-3421. 
74. Kuo, W.H., et al., Surface modification with poly(sulfobetaine methacrylate-co-acrylic acid) to reduce fibrinogen adsorption, platelet adhesion, and plasma coagulation. Biomacromolecules, 2011. 12(12): p. 4348-56.

75. Kikuchi, A., et al., Adsorbed serum protein mediated adhesion and growth behavior of bovine aortic endothelial cells on polyamine graft copolymer surfaces. J Biomater Sci Polym Ed, 1996. 8(2): p. 77-90.

76. Goddard, J.M. and J.H. Hotchkiss, Polymer surface modification for the attachment of bioactive compounds. Progress in Polymer Science, 2007. 32(7): p. 698-725.

77. Altankov, G., K. Richau, and T. Groth, The role of surface zeta potential and substratum chemistry for regulation of dermal fibroblasts interaction. Materialwissenschaft und Werkstofftechnik, 2003. 34(12): p. 1120-1128.

78. Zhu, Y. and Y. Sun, The influence of polyelectrolyte charges of polyurethane membrane surface on the growth of human endothelial cells. Colloids and Surfaces B: Biointerfaces, 2004. 36(1): p. 49-55.

79. van Wachem, P.B., et al., Adhesion of cultured human endothelial cells onto methacrylate polymers with varying surface wettability and charge. Biomaterials, 1987. 8(5): p. 323-8.

80. Berthelemy, N., et al., Polyelectrolyte Films Boost Progenitor Cell Differentiation into Endothelium-like Monolayers. Advanced Materials, 2008. 20(14): p. 2674-2678.

81. Tziampazis, E., J. Kohn, and P.V. Moghe, PEG-variant biomaterials as selectively adhesive protein templates: model surfaces for controlled cell adhesion and migration. Biomaterials, 2000. 21(5): p. 511-520.

82. Bornstein, P. and E.H. Sage, Matricellular proteins: extracellular modulators of cell function. Current Opinion in Cell Biology, 2002. 14(5): p. 608-616.

83. Mann, B.K., et al., Modification of surfaces with cell adhesion peptides alters extracellular matrix deposition. Biomaterials, 1999. 20(23-24): p. 2281-2286.

84. Fei, J., Z. Zhang, and L. Gu, Bending behaviour of electroresponsive poly(vinyl alcohol)/poly(acrylic acid) semi-interpenetrating network hydrogel fibres under an electric stimulus. Polymer International, 2002. 51(6): p. 502-509.

85. Delcroix, M.F., et al., Design of Mixed PEO/PAA Brushes with Switchable Properties Toward Protein Adsorption. Biomacromolecules, 2012. 14(1): p. 215-225.

86. Donald L.Jacobs, J.B.T., Hemostasis and coagulation, in Vascular Surgery: Basic Science and Clinical Correlations, M. Rodney A. White, Larry H. Hollier, MD, Editor. 2008, John Wiley \& Sons.

87. Kottke-Marchant, K., The Role of Coagulation in Arterial and Venous Thrombosis, in Antithrombotic Drug Therapy in Cardiovascular Disease, A.M.L. Arman T. Askari, Editor. 2010.

88. Geenen, I.L., et al., Endothelial cells (ECs) for vascular tissue engineering: venous ECs are less thrombogenic than arterial ECs. J Tissue Eng Regen Med, 2012.

89. Raymond, J., A.C. Desfaits, and D. Roy, Fibrinogen and Vascular Smooth Muscle Cell Grafts Promote Healing of Experimental Aneurysms Treated by Embolization. Stroke, 1999. 30(8): p. 1657-1664.

90. Kvanta, A., et al., Expression of Matrix Metalloproteinase-2 (MMP-2) and Vascular Endothelial Growth Factor (VEGF) in Inflammation-associated Corneal Neovascularization. Experimental Eye Research, 2000. 70(4): p. 419-428.

91. De Rosa, M., et al., Cationic polyelectrolyte hydrogel fosters fibroblast spreading, proliferation, and extracellular matrix production: Implications for tissue engineering. Journal of Cellular Physiology, 2004. 198(1): p. 133-143. 
92. Heydarkhan-Hagvall, S., et al., Co-culture of endothelial cells and smooth muscle cells affects gene expression of angiogenic factors. Journal of Cellular Biochemistry, 2003. 89(6): p. 1250-9.

93. Patel, A., et al., Elastin biosynthesis: The missing link in tissue-engineered blood vessels. Cardiovascular research, 2006. 71(1): p. 40-9.

94. Food and Drug Administration, Device Approvals and Clearances. http://www.fda.gov/MedicalDevices/ProductsandMedicalProcedures/DeviceApprov alsandClearances/PMAApprovals/ucm311892.htm, May 2012.

95. Khutoryanskaya, O.V., et al., Hydrogen-Bonded Complexes and Blends of Poly(acrylic acid) and Methylcellulose: Nanoparticles and Mucoadhesive Films for Ocular Delivery of Riboflavin. Macromolecular bioscience, 2014. 14(2): p. 225-34.

96. Serrano, M.C., et al., In vitro biocompatibility assessment of poly ( $\varepsilon$-caprolactone) films using L929 mouse fibroblasts. Biomaterials, 2004. 25(25): p. 5603-5611.

97. Murdan, S., Electro-responsive drug delivery from hydrogels. Journal of Controlled Release, 2003. 92(1-2): p. 1-17. 


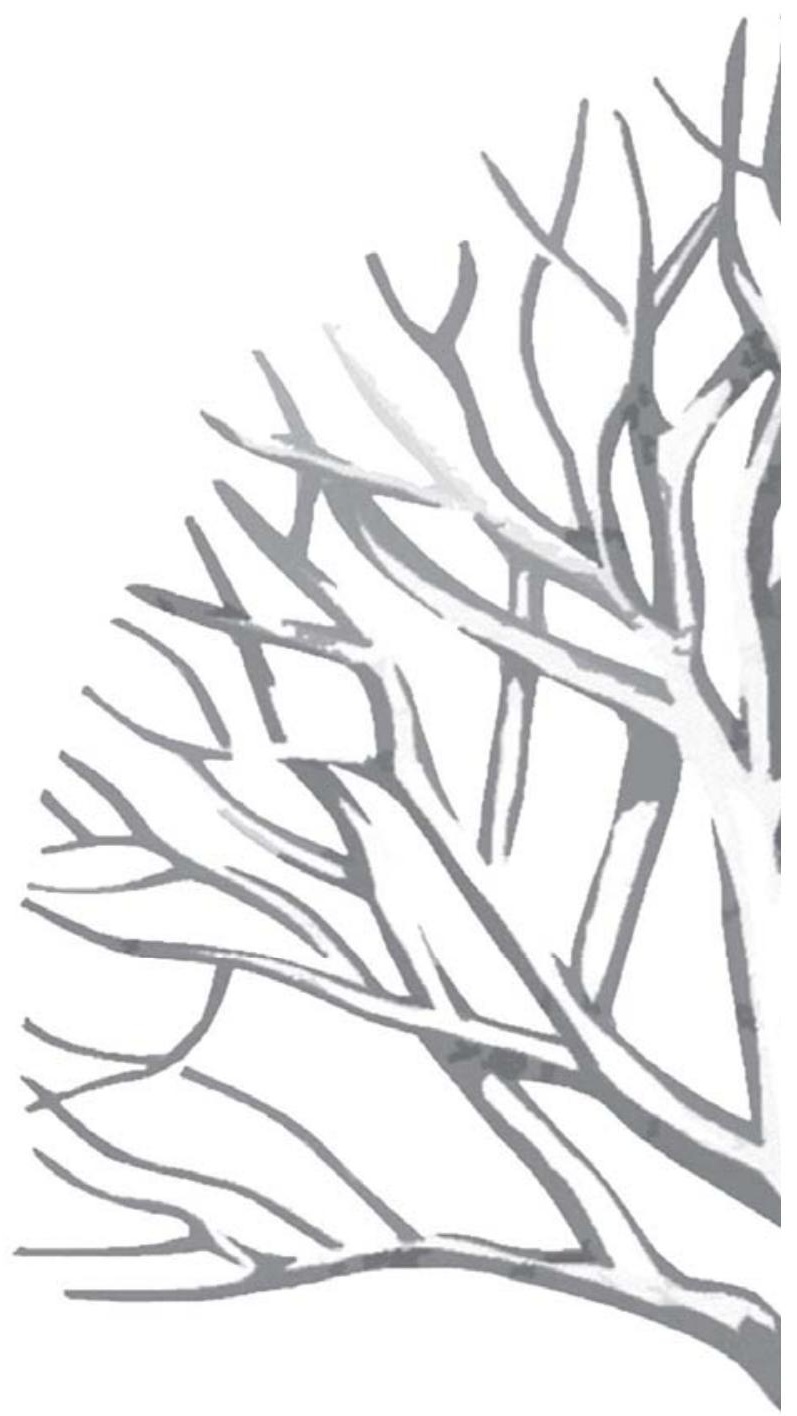




\section{Summary}

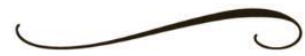

Vascular tissue engineering is an advanced therapeutic approach to provide appropriate alternative grafts for blood vessel transplantation in patients with cardiovascular diseases and in particular coronary artery disease. Tissue engineering combines the engineering and biological methodologies to regulate cell behavior and tissue regeneration. Smartly designed biomaterials in combination with external stimuli provide essential cues to the physiological microenvironment of the cell to direct its position and function in tissue engineered constructs. In this study, we employed such a biomaterial, in particular one that responds to an external electrical field.

Electro-responsive hydrogels hold great potential as smart substrates and we hypothesized that application of these hydrogels improves cell function and results in desired cell seeding behavior and tissue formation upon application of electric stimuli.

A hybrid electro responsive hydrogel was designed and manufactured by combining Poly acrylic acid and Fibrin. Characterization of the prepared samples by FTIR-ATR, scanning electron microscopy and swelling measurement confirmed the incorporation of Fibrin in the hydrogel network. Electrical stimulation ( $0.06 \mathrm{v} / \mathrm{mm}, 0.0167 \mathrm{~Hz}, 2 \mathrm{hrs}$ ) resulted in continues bending, produced by hydrogel swelling/ deswelling. The gel was biocompatible with no cytotoxic effects on smooth muscle cells (SMCs). Electrical stimulation of PAA/Fibrin hydrogels seeded with SMCs caused Fibrin fibers and smooth muscle actin filaments to align due to swelling/deswelling of the hydrogel. Additionally, penetration of cells into the depth of the hydrogel was significantly enhanced in the stimulated hydrogels compared with non-stimulated samples. In the presence of the voltage gated calcium channel blocker Verapamil, SMCs still aligned and penetrated into the gel, suggesting that observed effect was not due to a direct effect of electrical stimulation on the cells.

We subsequently investigated if the PAA/Fibrin biomaterial in combination with alternating electrical stimulation supported long-term collagen production and remodeling of the tissue construct. Seeded hydrogels were subjected to an electrical field $(0.06 \mathrm{v} / \mathrm{mm}, 0.0167 \mathrm{~Hz}, 2 \mathrm{hrs})$ once, twice or three times a week during 4 weeks of culturing and compared with non-stimulated control condition. Collagen morphology and production were assessed by CNA-35 probe and two-photon laser scanning microscopy. Electrical stimulation resulted in 
fibrillar collagen network whereas non-stimulated samples showed local spot deposition of collagen in the structure. As expected from the cell distribution collagen production in the center of the hydrogels was increased by more than 100 fold ( $1 \mathrm{x}$ stimulation/wk) after 4 weeks of culture compared with controls. Such an increase was further confirmed by higher collagen gene expressions in the stimulated samples by PCR analysis. The enhanced collagen production and distribution resulted in significantly improved mechanical properties of the hydrogels. Elevated Matrix Metalloprotease II (MMP2) activity in stimulated samples suggests a role in reorganization of the collagen in the stimulated samples.

We hypothesized that the PAA/Fibrin scaffold in combination with electrical stimulation might change SMC phenotype other than stimulating them to produce more collagen. Western Blot analysis of the samples revealed increased SMMHC, Calponin and SM- $\alpha$ actin proteins expression in electrically stimulated PAA/Fibrin hydrogels, indicating a phenotype transition of SMCs into a more contractile state together with the increased collagen production this suggests a dual phenotype pattern where some SMC are contractile while others are synthetic (i.e. collagen producing).

Further exploring the mechanism of alignment, in response to electrical stimulation, we studied the same electrical conditions in a PAA gel only (without Fibrin). Conversely, we subjected PAA/Fibrin gel to cyclic stretch equivalent to the induced mechanical change by the electrical stimulus (Flexcell: $5 \%$ stretch, $0,0167 \mathrm{~Hz}, 2 \mathrm{hrs}$ ). In the absence of Fibrin, electrical stimulation of the SMC in PAA gel SMC no longer aligned in spite of the induced mechanical force of the gel. This indicates that the deformation of Fibrin by electrical shape change of PAA is crucial for the observed effects on SMCs. Mechanical stimulation in the Flexcell apparatus did not affect SMC phenotype either. Together, these results suggest that both mechanical alignment of Fibrin and electrical stimulation are important for the observed changes in SMC phenotype.

The enhanced cell penetration and phenotypic SMC changes prompted us to apply the PAA/Fibrin gel as coating of an electrospun PCL graft. Grafts were implanted as interposition graft in a rat abdominal aorta. Rats were exposed to electrical stimulation $\left(1 \mathrm{~mW} / \mathrm{cm}^{2}, 0.0167 \mathrm{~Hz}\right)$ in ventro-dorsal axis (1 hour) followed by 1 hour in bi-lateral axis, once per week during 4 and 8 weeks of implantation. Non-stimulated rats with PAA/fibrin/PCL and PCL grafts served as controls. Angiography and ultrasound showed $100 \%$ open lumen of stimulated and non-stimulated PAA/Fibrin/PCL grafts in 4 weeks and 8 weeks of implantation. In the non-stimulated grafts 3 out of 12 grafts developed an 
aneurysm versus $0 / 12$ in the stimulated group. Immunohistochemistry images of explanted vascular grafts after 4 and 8 weeks revealed higher cellular density in the stimulated grafts compared to non-stimulated ones. Stimulation of grafts also resulted in enhanced endothelial coverage during the first 4 weeks of implantation as judged by $C D 31$ immunopositivity. CD 68 staining showed no significant difference in the foreign body reaction between stimulated and nonstimulated grafts. Promising results of electrically stimulated hybrid grafts indicated high potential of our proposed system for small-diameter vascular grafts development.

A general discussion concludes the body of work by integrating the obtained results in the research study with reference to existing evidence. We conclude that cell behavior and tissue formation in tissue engineered constructs can by controlled by micro and macro scale deformations of electro-responsive hydrogels subjected to electrical field. The proposed system has a high potential as a multi-functional smart biomaterial for biomedical applications. 


\section{Samenvatting}

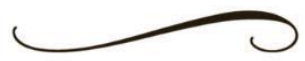

Tissue engineering van bloedvaten is een geavanceerde medische technologie die tot doel heeft om patiënt-eigen vaten te vervangen als graft bij diverse vormen arteriële obstructie. In tissue engineering worden chemisch-technische, fysische en biologische methoden gecombineerd om cellen te sturen zodat ze optimaal het beoogde weefsel nabootsen. Slimme biomaterialen al of niet in samenwerking met externe stimuli blijken in staat om de ligging, oriëntatie en functie van cellen te kunnen beïnvloeden. In dit project, bestaande uit een viertal studies, hebben we zo'n biomateriaal gebruikt en gekeken naar de effecten van elektrische stimulatie op gedrag van gladde spiercellen genomen uit de vaatwand.

Biomaterialen die reageren op elektrische stimuli zijn veelbelovend en we hebben daarom onderzocht of deze materialen in staat zijn om cel distributie en functie te sturen en of daarbij weefsel productie wordt verbeterd of bespoedigd. Een hybride hydrogel van Poly-acryl zuur en Fibrine die reageert op een externe elektrische spanning werd ontworpen en gemaakt. De hydrogel werd gekarakteriseerd met Fourier Transformed Infra Red spectroscopy gebruikmakend van attenuated total reflectance (FITR-ATR) en met scanning electron microscopy (SEM). Elektrische stimulatie $(0.06 \mathrm{~V} / \mathrm{mm}, 0.0167 \mathrm{~Hz}$ ) gedurende 2 uur veroorzaakte oscillerende verbuiging van de hydrogel als gevolg van alternerende zwelling en krimp. De gel was biocompatibel en niet toxisch voor cellen. In aanwezigheid van gladde spiercellen veroorzaakte de verbuiging van de hydrogel een parallelle ligging van Fibrine vezels en cellen. De ligging van de cellen werd vastgesteld door visualisatie van intracellulaire actine vezels. Tevens zorgde de elektrische stimulatie van de hydrogel voor betere penetratie van de cellen in de gel. Dit was onafhankelijk van elektrische activatie of contractie van de cellen aangezien Verapamil, een blokker van voltage afhankelijke Calcium kanalen, de geobserveerde effecten niet inhibeerde.

Vervolgens werd onderzocht of de verbeterde penetratie en de parallelle oriëntatie van de gladde spiercellen ook leidt tot betere weefsel productie, meestal gekwantificeerd aan de hand van collageen productie. Hiertoe werden de hydrogelen gezaaid met gladde spiercellen, vervolgens al of niet elektrisch gestimuleerd, en 4 weken gekweekt zodat weefsel vorming kon plaatsvinden. De stimulatie vond 1, 2, of 3 keer per week plaats om het effect van totale stimulatie duur te testen. De hoeveelheid geproduceerd collageen en de structuur van geproduceerde collageen werd onderzocht met behulp van 
beeldvorming (twee foton laser scanning microscopie), waarbij collageen werd aangekleurd met een CNA-35 eiwit dat specifiek bindt aan fibrillair collageen. Elektrische stimulatie resulteerde in een netwerk van fibrillair collageen terwijl de niet gestimuleerde gekweekte hydrogelen alleen locale niet gestructureerde eilandjes van collageen vertoonden. Als te verwachten op grond van de diepere cel penetratie in de gel was ook de collageen depositie dieper in de gel aanwezig (1x stimulation/wk), tot zelfs 100 keer zoveel als in niet-gestimuleerde hydrogelen. De grotere hoeveelheid collageen was deels het gevolg van verhoogde productie aangezien door PCR analyse een hogere transcriptie van het collageen eiwit werd aangetoond. Fibrillair collageen verleent stevigheid aan het weefsel en de stimulatie leidde dan ook tot significant sterkere weefsels, waarneembaar in mechanische testen. Afbraak van collageen gebeurt door een set enzymen, metalloproteinases genoemd. In gladde spiercellen is MMP-2 de belangrijkste. MMP-2 activiteit werd ook verhoogd door elektrische stimulatie wat er op duidt dat het neergelegde collageen actief wordt gereorganiseerd, bijvoorbeeld om een betere onderlinge structuur te kweken.

Andere gladde spiercel eigenschappen kunnen ook veranderen door kweek in de PAA/Fibrine hydrogelen die elektrisch worden gestimuleerd. Dit werd getest door te kijken naar de eiwit expressie van smooth muscle myosin heavy chain (SMMHC), Calponin en Smooth muscle alpha actin (SM- $\alpha$ ) met behulp van Western Blot analyse. Het bleek dat deze eiwitten ook werden gestimuleerd door de elektrisch geïnduceerde, mechanische vervorming van de PAA/Fibrine hydrogel, wat suggereert dat deze gladde spiercellen een meer contractiel fenotype gingen vertonen terwijl in het totale weefsel ook meer collageen werd geproduceerd, duidend op een synthetisch fenotype. In afwezigheid van Fibrine, dus alleen in de PAA gel werd geen effect van elektrische stimulatie op gladde spiercel fenotype waargenomen. Ook gaf cyclische mechanische rek van de PAA/Fibrine zonder elektrische stimulatie geen fenotypische responsen te zien. Dit betekent dat de vervorming van Fibrine door de elektrische geïnduceerde verandering van de PAA cruciaal is voor de geobserveerde effecten en dat de juiste parallelle oriëntatie van Fibrine noodzakelijk is de veranderingen in het fenotype van de gladde spiercel.

Verbeterde penetratie van gladde spiercellen in de hydrogel en hun fenotypische verandering in contractiele cellen zijn goede eigenschappen bij vaatwand genezing na stent plaatsing. In eerdere studies was een vasculaire graft getest die gemaakt is uit elektro-gesponnen Polycaprolacton draden. We veronderstelden dat coating van de $P C L$ draden met PAA/Fibrine gel de ingroei van contractiele gladde spiercellen zou bevorderen en daarmee de intima 
hyperplasie zou verminderen. Intima hyperplasie is een woekering van synthetische gladde spiercellen die het gestente bloedvat opnieuw vernauwd. De PAA/Fibrine gecoate PCL grafts werden geplaatst als interpositie grafts in de abdominale aorta van de rat. Ze werden transcutaan gestimuleerd met een elektrisch veld dat lokaal dezelfde sterkte had als in de in vitro studies. De follow up was 4 of 8 weken en gedurende de gehele periode werden ze eens per week gestimuleerd. Na 4 en 8 weken waren alle grafts open bij angiografische en echografische controle. Drie van de 12 niet gestimuleerde grafts hadden een aneurysma terwijl geen van de gestimuleerde grafts aneurysmatisch was. Zoals in vitro leidde stimulatie tot betere ingroei van gladde spiercellen en na 4 weken was ook de regeneratie van endotheel verhoogd. De vreemd lichaam reactie was gelijk in de groepen.

Deze veelbelovende resultaten met de PAA/Fibrine hydrogel die reversibel vervormd door elektrische stimulatie laten zien dat hybride hydrogelen uitstekend gebruikt kunnen worden in diverse regeneratieve geneeskunde applicaties. De ontwikkeling van een kleine diameter vasculaire graft is een van de mogelijkheden.

In een algemene discussie worden de resultaten van de vier studies geïntegreerd en gerelateerd aan vergelijkbare studies in de literatuur. Ik concludeer dat het gedrag van cellen en de resulterende weefselvorming gestuurd kan worden door reversibele vervormingen op micro en macro schaal van een elektrisch veld gevoelige hydrogel. Dit hybride biomateriaal heeft grote mogelijkheden als een multifunctioneel slim biomateriaal voor biomedische toepassingen. 
Valorization

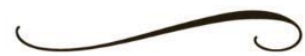

\section{Clinical need}

Cardiovascular disease (CVD) is one of the major causes of morbidity and mortality in the world. In 2012, 27\% of the deaths in the Netherlands were related to CVD of which $54 \%$ was related to coronary artery disease (Central Bureau Statistiek, The Hague / Heerlen). Although the numbers have decreased over the past decade ( $6 \%$ decrease compared to 10 years ago), the social and economic burden still remains high [1].

A high clinical need of alternative treatments for blood vessel dysfunction and pathology, representing the majority causes underlying CVDs, has developed as basic treatment improved life expectancy. In 2012, about $67 \%$ of the adult cardiac operations in the Netherlands involved coronary artery bypass procedure and the usage of arterial grafts in artery bypass surgeries has been reported to be approximately $26 \%$ in 2011 , with $11 \%$ increase compared to 1995 [1].

In patients with coronary artery disease, the inner lumen diameter of coronary arteries that allows transportation of highly oxygenated blood into the heart becomes narrowed due to atherosclerotic plaque formation inside the vessel wall. The hardening or the potential rupture of these plaques over time will block blood flow to the heart muscle and can lead to chest pain, myocardial infarction and potentially death. Coronary artery bypass surgery is a treatment to restore the blood flow to the heart by replacing the damaged vessel with a healthy functional substitute. Peripheral vascular disease can also be caused by atherosclerosis with consequential, narrowing or blocking of the vessel and reduced blood flow of the peripheral system. Therefore, developing appropriate and more cost-effective alternatives is an urgent need in the field.

Autologous blood vessels are considered the gold standard and main material for grafting, yet the application of these vessels is restricted by the clinical condition of the patient and limited number of suitable vascular substitutes due e.g. amputation or earlier usage for grafting [2]. Furthermore, the required graft dimension is not always available and harvesting of blood vessels comes with a high risk of necrosis at the site of the surgery [3]. Clinically successful synthetic grafts are commercially available with diameters above $6 \mathrm{~mm}$, using Dacron (polyethylene terephthalate) or expanded polytetraflouroethylene (ePTFE) [4, 5]. However, these biomaterials frequently fail as small diameter $(<6 \mathrm{~mm})$ vascular graft due to developing microfractures and thrombus formation after 
implantation. Small diameter blood vessels replacements are required for treating coronary artery and peripheral vascular diseases in the distal part of the limb circulation [6].

Tissue engineering is a promising strategy to develop alternative substitutes for replacement of diseased blood vessels. By providing biomaterial grafts to these patients tissue perfusion could restore blood flow to the heart or legs and therefore increase the chance of patients to survive and regain quality of life. However, despite all research and clinical attempts, producing small diameter blood vessels is still challenging. To date, there is no commercially available small diameter vascular graft (diameter less than $5 \mathrm{~mm}$ ) to be used as substitute for autologous vein transplantation in coronary artery disease. Therefore, introducing novel biomaterials and tissue regeneration therapies could be of interest for medical device manufacture companies looking for innovative approaches in patients with CVD.

\section{Societal conditions}

The considerable cardiovascular mortality and prevalence of cardiac disease over the years are not only affecting human health but also greatly influences economic stability in the world [2]. For instance, cardiovascular diseases currently take up $17 \%$ of the overall national health care costs in United states [7]. In 2012, European sales of vascular grafts amounted to approximately $\$ 865$ million and will reach around $\$ 1.3$ billion in 2017 according to Medtech Insight report [8].

Tissue engineering blood vessels have been shown to be socially and economically effective when native vessel replacements are not available [9]. Using tissue engineered (biomaterial) blood vessels instead of native veins would eliminate the complications of harvesting veins (which reduce the treatment costs by more than $\$ 5,000$ per patient) as well as reducing the high risk of early thrombosis occurring in autologous grafts likely caused by endothelial layer damage during harvesting procedure. Thrombosis can be reduced by pre-endothelialization of the grafts [9]. Additionally, tissue engineered vascular grafts could be modified to contain various functional substrates, which could facilitate treatment and regeneration of the tissue. This advantage over autologous vein grafts could have major economic advantage by reducing the healing time.

Risk of graft rejection for a tissue engineered product is presumably lower than for xenografts and indeed is considered to be also less than traditional 
treatments as it is essentially a customized process and not subject to mass-scale production [10]. However, tissue engineering still involves risks as the process includes manipulation of cells and tissues. The nature of risks associated with tissue engineered grafts has been described in detail by the European commission health and consumer protection report [11]. Briefly, the risk factors include microbiological contamination of the biomaterial and the scaffold, nonbiological contamination during the process, delivery of un-wanted cells, modification of the cells during the process, sterility of the final product, functionality of the final regenerated tissue, unknown risks associated with unwanted reaction of cells and scaffold and a foreign body reaction or immunological rejection of the patient [11].

\section{Product}

The results of the presented research could be introduced into the clinic by producing small diameter vascular grafts from smart electro-responsive biomaterials, which can be externally controlled by applying an electrical field. The external electrical field application should be designed to be patient friendly and non-invasive. An external electrical field has already been applied for healing of wounds and fractured bones. In the animal study performed in this research, electro-responsive hydrogels were used as coating to functionalize electro-spun small diameter $\omega$-polycaprolacton grafts. The electro-responsive hydrogel could be used in combination with other biopolymers as well. In addition, such a graft could also serve as a multifunctional product by incorporating drug delivery features into the system.

\section{Novelty of the concept}

According to our knowledge the proposed approach is novel in the vascular graft area. The presented research in this thesis shows the feasibility of electroresponsive hydrogels to improve cell distribution and collagen production within the structure in vitro as well as improving the integration of an implanted small diameter vascular graft and the recipient artery in vivo. In the animal study, the external electrical stimulation facilitated cell infiltration into the graft and reduced neo-intima formation. This method could be a promising approach in developing tissue engineered constructs for clinical treatments in the future.

Shortening the time between implanting the vascular graft in the recipient body and the complete regeneration process could lead to economic improvement by 
reducing the after-surgery costs. The capability of the system for releasing preimbedded biomolecular factors in the graft structure is a promising approach in developing more bioactive tissue engineered vascular substitutes. For instance, essential regulatory factors focusing on reducing thrombosis could be controlled upon application of the external electrical field, which could improve the bloodbiomaterial interactions and facilitate tissue regeneration.

\section{Road to market}

The translation of the developed system from bench to bedside needs further in vitro and pre-clinical animal studies. Underlying cellular and molecular mechanism involved in the regeneration event could help us better understand the healing process and thereby improve the treatment strategy by reducing the host response to the implanted vascular graft. Whenever a new therapy or medical product is introduced to the health care market, the balance of risk and benefits must be carefully analyzed and studied [10]. It is essential to validate the scientific principle and process of the product prior to clinical use. Longer implantation period in animal models as well as full characterization of the explanted tissue needs to be performed and studied. Additionally, regulatory checks must be performed when the tissue engineered processes are introduced into clinical trials and finally, the market. Considering the wide variation of tissue engineering approaches, the regulatory factors needed for our proposed system need to be customized. The International Organization for Standardization (ISO) specifies the required properties for sterile vascular prostheses and the methods for testing the vascular prostheses intended to replace, bypass or to form shunts between segments of the vascular system in humans in ISO 7198 [12]. The requirements involve evaluation by visual Inspection, integral water permeability/leakage, longitudinal tensile strength, usable length, wall thickness, dynamic compliance, porosity, water entry pressure (WEP), burst strength, relaxed inner diameter, suture retention strength, water permeability, circumferential tensile strength, burst strength after repeated puncture, pressurized inner diameter, kink diameter / radius as well as packaging, labeling and terminology of the grafts [13]. It is important to prepare a full technical report of the product and system during the first clinical trial to describe the level of safety and provide evidence for improvement of the treatment compared to conventional therapies. The clinical trials must be conducted in clinical trials unit (CTU) that are regulated by clinical trials application (CTA) [14]. Additionally the product needs to meet Good Manufacturing Practices (GMP) 
guidelines to be authorized for human applications. These regulations are carried out by regulatory agencies such as the FDA, WHO or European Union's GMP (EU-GMP). Additionally, the medicinal product needs to be certified and its manufacturing must be licensed according to investigational medicinal product (IMP) regulations.

These processes could take up to 15 years and the costs to comply with the evaluations and establish safety will be part of starting-up the process.

In conclusion, our research study provided proof of concept for using electroresponsive hydrogels as smart biomaterials for building scaffolds and small diameter vascular grafts. Our promising results on improving cell behavior in interaction with the electrically stimulated structure could introduce new opportunities for development of vascular grafts in regenerative medicine.

\section{References:}

1. Leening, M.J., S. Siregar, I. Vaartjes, M.L. Bots, M.I. Versteegh, R.J. van Geuns, J.J. Koolen, and J.W. Deckers, Heart disease in the Netherlands: a quantitative update. Netherlands heart journal : monthly journal of the Netherlands Society of Cardiology and the Netherlands Heart Foundation, 2014. 22(1): p. 3-10.

2. Nemeno-Guanzon, J.G., S. Lee, J.R. Berg, Y.H. Jo, J.E. Yeo, B.M. Nam, Y.-G. Koh, and J.I. Lee, Trends in Tissue Engineering for Blood Vessels. Journal of Biomedicine and Biotechnology, 2012. 2012: p. 14.

3. Polymer Grafting and Crosslinking, in Polymer Grafting and Crosslinking, J.W.R. Amit Bhattacharya, Paramita Ray, Editor 2009, John Wiley \& Sons, Inc.,: New Jersey.

4. Chlupac, J., E. Filova, and L. Bacakova, Blood vessel replacement: 50 years of development and tissue engineering paradigms in vascular surgery. Physiological research / Academia Scientiarum Bohemoslovaca, 2009. 58 Suppl 2: p. S119-39.

5. Kannan, R.Y., H.J. Salacinski, K. Sales, P. Butler, and A.M. Seifalian, The roles of tissue engineering and vascularisation in the development of micro-vascular networks: a review. Biomaterials, 2005. 26(14): p. 1857-75.

6. Isenberg, B.C., C. Williams, and R.T. Tranquillo, Small-Diameter Artificial Arteries Engineered In Vitro. Circulation Research, 2006. 98(1): p. 25-35.

7. Catto, V., Far, \#xe8, S. , G. Freddi, and M.C. Tanzi, Vascular Tissue Engineering: Recent Advances in Small Diameter Blood Vessel Regeneration. ISRN Vascular Medicine, 2014. 2014: p. 27.

8. U.S. Markets, Trends, and Opportunities in Patient Monitoring Products, Report \#A349 November 2013.

9. L'Heureux, N., N. Dusserre, A. Marini, S. Garrido, L. de la Fuente, and T. McAllister, Technology Insight: the evolution of tissue-engineered vascular grafts[mdash]from research to clinical practice. Nat Clin Pract Cardiovasc Med, 2007. 4(7): p. 389-395.

10. Williams, D., Benefit and risk in tissue engineering. Materials Today, 2004. 7(5): p. 24-29.

11. The Scientific Committee on Medicinal Products and Medical Devices, European commission health and consumer protection directorate-general, Opnion on the state of the art concerning tissue engineering. 2001. 
12. International Organization for Standardization (ISO), ISO 7198:1998-Cardiovascular implants -- Tubular vascular prostheses.

13. Biomedical Device Consultants \& Laboratories, L., http://www.bdclabs.com/medicaldevice-testing/vascular-graft-testing.php.

14. MHRA, Medicines and Healthcare Products Regulatory Agency, http://www.mhra.gov.uk/. 


\section{Acknowledgement}

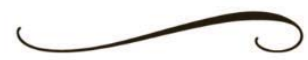

This is a great moment when you look back at the way and remember all those days, each as a step, building up the road and bringing you where you are now. Over the 4 years of my PhD I had the great chance to learn and experience lots of new things. All these were not definitely possible without the support and accompany of all the people who were by my side and contributed in many ways to the fulfillment of this study. Hereby I would like to thank all of you that made these years an unforgettable experience for me.

First and foremost, my deep and heartfelt gratitude goes to my supervisor Professor Mark Post. Mark, thank you so much for giving me the opportunity to be your PhD student. Thank you for all your inspiring guidance, support and encouragement during all the moments of these years. I could never imagine having a great supervisor like you when I was leaving Iran. Ever since, I learned so many things by your excellent supervision and your great personality. Thank you for giving me the space to develop whiles your insightful supervision was always, and is still, directing me. You made a major impact in my scientific life and I will never forget your great care and support in my personal life and my future career. I feel really proud of having the chance to work with you.

The second person who my sincere gratitude goes to is my co-supervisor Dr. Daniel Molin. Daniel, I am very grateful to you for all your constant help and support during my research study; from reviewing my manuscripts to setting up collaborations with other universities. Thank you for sharing your insights and knowledge and providing me with your helpful advices whenever I needed help both in my academic and personal life. I am also very thankful to you for giving me the opportunity to join Biomimedics program and establish an exchange of knowledge which greatly contributed in expanding my scientific network and collaborations.

I would also like to gratefully thank my committee members; Prof. Leo Koole, Prof. Christian Grandfils, Prof. Carlijn Bouten, Dr. Frans van Nieuwenhoven and Dr. Leon Schurgers for accepting to review my manuscript thesis.

Next, I would like to give a huge thank to my lovely colleagues in Physiology Department. Geertje, my dear paranymph, it was really great that I had you always by my side through all these years (not only morphemically but 
also literally that you were sitting next to me!). I want to thank you for all your generous helps in professional and personal supports. You are indeed the source of energy in the labs and of course in our office! I really had fun learning Western Blot and Zymography with you! Thank you for everything. Sanne, you were always very kind and supportive whenever I needed your help in the labs. Thank you for providing me all your technical inputs and assistance when I had to do PCR of my hydrogels. Allard, I would like to thank you for all your support in implanting my grafts into rats and indeed adopting my protocols for electrically stimulating them! I was feeling always comfortable during our animal experiments as I knew you take care of the things. Hanneke and Viviane, I would also like to thank you for your great help in the animal study. Vincenza, I have missed you so much since you left to Leuven to follow your post-doc career. You have always been a true friend to me during all these years. I will never forget all the moral supports you gave me since the beginning. Thank you for all your helps my dear Cinzia. Mark, my dear paranymph, working close to you means lots of nice talks and fun. I was always feeling happy seeing you at our door raising your mug and asking for "coffee?". All our chats specially those ones we were speaking Dutch were really enjoyable! Thank you for all the things Soltan Marko! Irma and Felix (fluffy) you were great colleagues to me. Thank you for making the warm atmosphere in our working place and in the labs. I really enjoyed the meetings and conferences we went together! I will never forget kind support and sympathy of you both when the pass-controller was not letting me to checkin in Greece. Nynke, I would like to thank you for all your kind support and helpful feedbacks during my study. Mick, Anon and Ruud, you were always very nice and willing to help me whenever you were seeing me running lab to lab looking for something. Thank you for all your helps. Martyna, you were my first student and I really enjoyed working with you. I wish you the best in your future as you really deserve it.

A special thank goes to Vivian and Bianca who made a great help and support with every single administrative paper works in these years.

I would like to extend my gratitude to Prof. Marc van Zandvoort, Dr. Wim Engels and Jeroen Hameleers for all their support with two photon laser scanning microscopy and sharing their expertise during this study.

Prof. Thomas Cleij, Dr. Hanne Diliën and Rafel Dema, I am really thankful to you for helping and supporting me to perform my chemical experiments in Hasselt University. 
During all these years of my study I realized once again how lucky I am to have generous love and emotional support of my beloved freinds and family, without whom my life couldn't be so colorful and pleasant. The first person who really deserve a great thank is my dearest Leyla, my best and closest friend since so many years. Leyla, I cannot imagine how my life could have been without you. In all the ups and downs, I was always feeling safe as I knew I have you by my side helping me with all your heart. All the moments and minutes we shared together are unforgettable parts of my life. Thank you for all your love and support. Thank you for being my dearest Leyla.

Secondly, I want to thank all my dear friends in Maastricht who made here feel like home all these years. Nice friends that I could always count on them whenever I needed help. Ali, I am really happy that I found a great friend like you. Thank you for all your helps and support in these years. You are for sure a lifelong friend. Mehrdad, thanks for all your delicious cooking that was gathering all of us to spend a nice evening in your house. Maryam, Ehsan, Shayegh, Saba, Ali, Anahita, Tjeerd, Sanaz, Shuan, Sara and Nasim, you guys made my days in Maastricht so enjoyable and shiny! All the days and evenings we spend together are great and unforgettable memories for me. I am sure that our friendship will last forever. Hussein and Stephanie thank you for your great help and support, I will never forget how kind and supportive you were to me. Pegah and Siavash thanks for all your helps and care specially in early days of my arrival in Maastricht. I also want to thanks my dear friend Amir who passed away in a tragic car accident three years ago. Dear Dadash Amir I would never forget your great help and support since we met. May you rest in peace. Next, I also like to express my warm gratitude to my dear friends all over the world who their love and support stayed close to me despite we were being far away in distance. Thank you Mergoor, Marzie, Hengame, Meri, Niloofar, Fafa, Anna, Homa, Fifi and many others for being my lovely true friends. Mohammad you deserve special thanks for your great help in making the electrical switch I worked with in all the steps of my project! I am proud of having a smart friend like you!

Finally, I would like to express my deep heartfelt gratitude to my lovely family. My dearest Maman and Baba, I ran out of words whenever I think about all your endless love, support, care and encouragement through all my life. Without the motivation and strength you are giving me I would not have been in this place today. I love you so much and I feel really proud of being your daughter. Thank you for being always with me and supporting me unconditionally in every way. 
Thank you for all your bottomless love. You are my greatest inspiration in life.

Then I want to thank my wonderful brothers Ehsan and Amin for all their constant love and care through all my life. My dearest Ehsan and Amin I am so blessed of having you guys in my life!

I would also like to express my sincere gratitude to my second family and special thanks to my dear mother in-law and father in-law for all their love and affection. Rico, my sweet cute cat! You are indeed also a member of our family. Thank you for all the joy you brought to my life, for waking me up every morning and waiting for me all the evenings behind the window until I am back home. Most importantly, I end my acknowledgments with my loving husband, Ehsan. My dearest Ehsan no words can describe how your faithful love, support and warm encouragements enrich my spirit every day. Your love is my source of energy. Thank you for all your positive support and patience. Thank you for being my stronghold through all the ups and down. I feel really blessed of having you in my life my lovely Tala. 
Appendix 


\section{Curriculum Vitae}

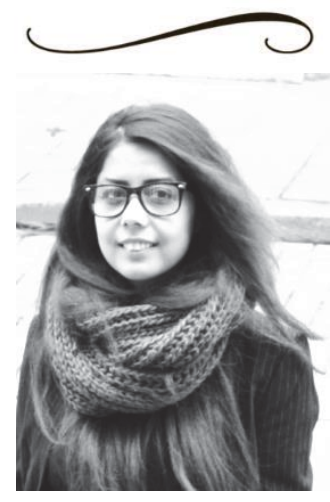

Nastaran Rahimi was born on May 8th, 1985 in Mashhad, Iran. After finishing her high school in 2004 in Mashhad, she attended "Amirkabir University of Technology (Polytechnic of Tehran)" where she obtained her Bachelor and Master of Science in Biomedical engineering, Biomaterials. In September 2009 she started working as a PhD student at the department of Physiology of Cardiovascular Research Institute Maastricht (CARIM) under supervision of Prof. Mark Post and Dr. Daniel Molin in Maastricht University of the Netherlands. The PhD project was funded for four years by the Biomedical Materials program (BMM) as part of the "iValve" project. In 2011, she received the award for best oral presentation at 4th tissue engineering conference in Crete, Greece. In September 2013, she joined the Biomimedics program of Euregional Meuse-Rhine Interreg IVa Project to continue her research in a postdoctoral position on medical application of novel biomaterials. 
Appendix 


\section{List of Publications}

4

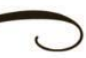

1. Nastaran Rahimi, Daniel G. Molin, Thomas J. Cleij, Marc A. van Zandvoort, Mark J. Post. Electrosensitive Polyacrylic Acid/Fibrin Hydrogel Facilitates Cell Seeding and Alignment. Biomacromolecules. Biomacromolecules, 13, 2012, p1448

2. Mark Post, Nastaran Rahimi, Vincenza Caolo, Update on vascularization in tissue engineering. Regenrative Medicine, 8, 2013, p759

3. Nastaran Rahimi, Geertje Swennen, Sanne Verbruggen, Martyna Scibiorek, Daniel Molin, Mark Post. Short stimulation of electro-responsive PAA/Fibrin hydrogel induces collagen production, Tissue engineering, 2014; 20(9):703-13

4. Nastaran Rahimi, Martyna Scibiorek, Geertje Swennen, Sanne Verbruggen, Daniel Molin, Mark Post. Modulation of smooth muscle cells morphology and phenotype by electro-responsive hydrogel, in progress

5. Nastaran Rahimi, Allard Wagenaar, Hanneke Cobelens, Daniel Molin, Mark Post. Vascular cell response in electro-responsive hybrid grafts by short stimulation: in vivo study, in progress 